\title{
Chiral control of spin-crossover dynamics in Fe(II) complexes
}

\author{
Malte Oppermann ${ }^{\star 1}$, Francesco Zinna ${ }^{2,3}$, Jérôme Lacour ${ }^{3}$, and Majed Chergui ${ }^{1}$ \\ ${ }^{1}$ Laboratoire de Spectroscopie Ultrarapide, École Polytechnique Fédérale de Lausanne, ISIC-FSB, 1015 Lausanne, \\ Switzerland \\ ${ }^{2}$ Dpto. di Chimica, Università di Pisa, Via Giuseppe Moruzzi 13, 56124 Pisa, Italy \\ ${ }^{3}$ Département de chimie organique, Université de Genève, Quai Ernest Ansermet 30, 1211 Genève 4, Switzerland \\ *malte.oppermann@epfl.ch
}

\section{ABSTRACT}

Iron-based spin-crossover (SCO) complexes hold tremendous promise as multifunctional switches in molecular devices. However, real-world technological applications require the excited high-spin (HS) state to be kinetically stable - a feature that has only been achieved at cryogenic temperatures in the light-induced excited spin-state trapping effect. Here we demonstrate HS state trapping by controlling the chiral configuration of Fe" 4,4 '-dimethyl-2,2'-bipyridine $)_{3}$ in solution, associated for stereocontrol with enantiopure $\Delta$ - or $\Lambda$-TRISPHAT anions. We characterize the HS state relaxation using a newly developed ultrafast circular dichroism technique in combination with transient absorption and anisotropy measurements. We find that the decay of the HS state is accompanied by ultrafast changes of its optical activity, reflecting the coupling to a symmetry-breaking torsional twisting mode, contrary to the commonly assumed picture. Furthermore, we show that the diastereoselective ion-pairing with the enantiopure anions suppresses the vibrational population of the identified twisting mode, thereby achieving a four-fold extension of the HS lifetime. Transferred to the solid state, this novel strategy may thus significantly improve the kinetic stability of iron(II)-based magnetic switches at room temperature.

The exquisite magnetic switching capabilities of transition metal complexes with energetically close-lying spin states are highly attractive for technological applications in information storage and processing ${ }^{1}$.

3 In this respect, $\mathrm{Fe}^{\mathrm{II}}$-based polypyridine complexes are particularly promising, due to their low cost and toxicity, earth-abundance, rich photochemistry and an efficient spin-crossover (SCO) transition that 5 can be triggered by various external stimuli including light, pressure and temperature ${ }^{2}$. However, the 6 excited paramagnetic spin states are notoriously short-lived at room temperature, which limits their use in molecular devices ${ }^{3}$.

The tris-chelate iron(II)tris(bipyridine) $\left(\left[\mathrm{Fe}^{\mathrm{II}}(\mathrm{bpy})_{3}\right]^{2+}\right)$ has emerged as a prototypical model complex, whose light-induced SCO mechanism serves as a reference for assessing the spin-switching dynamics of other $\mathrm{Fe}^{\mathrm{II}}$ complexes $^{4}$. To this end, ultrafast spectroscopies in the optical ${ }^{5-8}$ and X-Ray domain ${ }^{9-13}$ have $^{-}$ determined that the initially excited singlet metal-to-ligand charge-transfer (MLCT) state is deactivated 
in $<50$ fs with near unity efficiency via an essentially barrierless passage through the triplet MLCT and metal-centered (MC) states to the lowest-energy $\mathrm{MC}^{5} \mathrm{~T}_{2}$ state. The latter high-spin $(\mathrm{HS}, \mathbf{S}=2)$ state then converts back to the low-spin (LS, $\mathbf{S}=0){ }^{1} \mathrm{~A}_{1}$ ground state on the sub-nanosecond scale in aqueous solution. As the forward-SCO involves the transfer of two electrons from formally non-bonding $\mathrm{t}_{2 \mathrm{~g}}$ to antibonding $\mathrm{e}_{\mathrm{g}}$ ligand-field orbitals, the resulting weakening and elongation of the $\mathrm{Fe}-\mathrm{N}$ bonds by approximately $0.2 \AA$ has been established as the dominant reaction coordinate of the $\mathrm{SCO}^{3}$. Indeed, a so-called single reaction coordinate model has been proposed for $\mathrm{Fe}^{\mathrm{II}}-\mathrm{SCO}$ complexes, describing the non-radiative ${ }^{5} \mathrm{~T}_{2} \rightarrow{ }^{1} \mathrm{~A}_{1}$ back-SCO in a framework akin to semi-classical Marcus theory ${ }^{14,15}$, with the structural re-organization proceeding via a symmetric breathing mode of the Fe-N bonds and the electronic coupling mediated by the (thermally inaccessible) ${ }^{3} \mathrm{~T}_{1}$ state. In this picture, thermal HS deactivation is suppressed at sufficiently low temperatures, famously known as the light-induced spin-state trapping (LIESST) effect ${ }^{16}$.

However, it is now well established that the single reaction coordinate model fails to describe the SCO dynamics of $\mathrm{Fe}^{\mathrm{II}}$ systems with constrained ligand systems, where anisotropic expansions of the Fe-N bond network couple to dynamic angular distortions of the primary coordination sphere ${ }^{4,13,17-20}$. Importantly, it has been suggested that it is precisely the deviations from a single active Fe-N breathing coordinate that may provide a route to spin-state trapping at room temperature, as the coupling of the spin-relaxation to a distorted coordination environment can be manipulated through the mechanical properties of the ligand system ${ }^{21,22}$. Perhaps surprisingly, it has long been speculated that such distortions may also play a role in the SCO mechanism of unconstrained tris-chelate complexes, such as $\left[\mathrm{Fe}^{\mathrm{II}}(\mathrm{bpy})_{3}\right]^{2+}$, due to a fundamental link between the SCO process and their chiral three-bladed propeller structure: the entropydriven racemization of enantiopure or enantioenriched solutions at room temperature (RT) is accompanied by a change in spin-state ${ }^{23}$. This was rationalized at the end of the 1970s through calculations by Purcell and Vanquickenborne ${ }^{24,25}$, showing that the low-frequency torsional twisting modes associated with the racemization result in a surface crossing of the ${ }^{1} \mathrm{~A}_{1}$ and ${ }^{5} \mathrm{~T}_{2}$ states, whilst stabilizing the ${ }^{3} \mathrm{~T}_{1}$ state and thus increasing the associated spin-orbit coupling term. However, despite an early synthetic design study correlating the HS deactivation barrier with the flexibility of a hexadentate ligand framework along a torsional twisting coordinate ${ }^{26}$, conclusive experimental evidence for the fundamental role of this reaction 


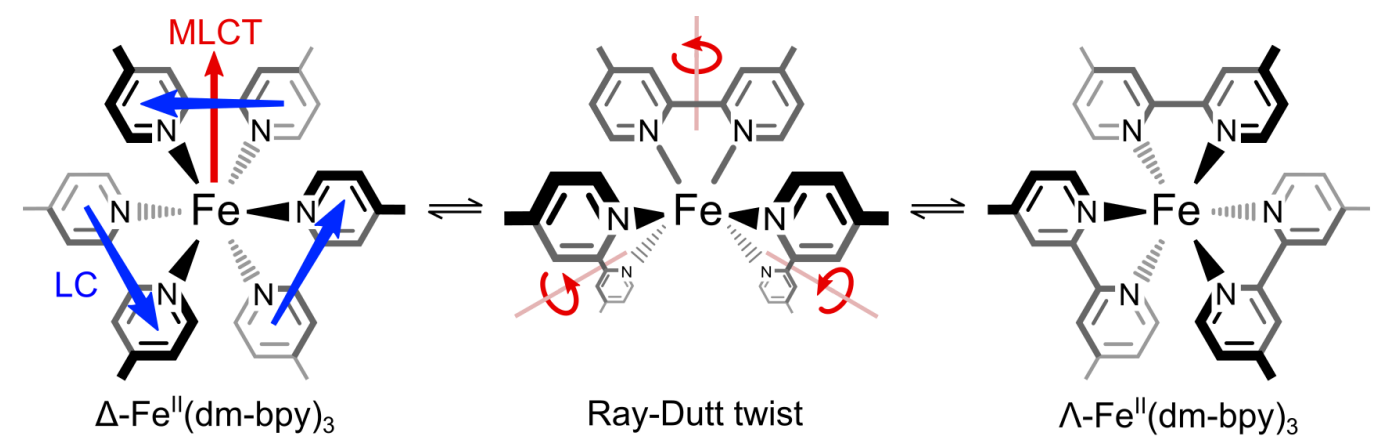

Figure 1. Due to the lability of its Fe-N bonds, an enantiomerization of the $\Lambda$ and $\Delta$ configurations of $\mathrm{Fe}^{\mathrm{II}}(\mathrm{dm} \text {-bpy })_{3}$ occurs in solution via the intramolecular Ray-Dutt twist. Molecular structure of $\Lambda, \Delta-\mathrm{Fe}^{\mathrm{II}}(\mathrm{dm}-\mathrm{bpy})_{3}$, including its metal-to-ligand charge transfer (MLCT) and ligand-centred (LC) long-axis $\pi \pi^{*}$ transition dipoles. The Ray-Dutt twist proceeds via a transition state structure with $\mathrm{C}_{2 \mathrm{v}}$ symmetry.

coordinate in $\mathrm{Fe}^{\mathrm{II}}$-based $\mathrm{SCO}$ complexes is still lacking.

In this study we reverse the perspective developed by Purcell and Vanquickenborne and investigate whether suppressing the enantiomerization of $\left[\mathrm{Fe}^{\mathrm{II}}(\mathrm{dm}-\mathrm{bpy})_{3}\right]^{2+}\left(\mathrm{dm}-\mathrm{bpy}=4,4^{\prime}\right.$-dimethyl-2,2'-bipyridine $)$ in RT solutions kinetically stabilizes the HS state. Fig. 1 displays the chiral structure of the three-bladed propeller complex with $\Lambda$ and $\Delta$ configurations and the transition dipoles for its singlet MLCT and ligand-centered (LC) long-axis $\pi \pi^{*}$ transitions. As other hexacoordinated tris-chelate complexes, the enantiomerization process may proceed via two possible non-dissociative pathways, which pass through trigonal prismatic transition states with different symmetries: the Bailar twist via a $\mathrm{D}_{3 \mathrm{~h}}$ and the Ray-Dutt twist via a $\mathrm{C}_{2 \mathrm{v}}$ symmetry (see fig. 1). Whilst the dominant mechanism depends on both the metal center and the ligand system, a recent DFT study on $\left[\mathrm{Fe}^{\mathrm{II}}(\mathrm{bpy})_{3}\right]^{2+}$ found the Ray-Dutt twist to be energetically favorable, both on the singlet and the quintet surface ${ }^{27}$. Due to the lability of the Fe-N bonds, torsional twists have a low kinetic barrier in RT solutions, resulting in the formation of racemic mixtures with equal amounts of $\Lambda$ and $\Delta$ enantiomers and no detectable optical activity. However, diastereomeric interactions with enantiopure counterions (TT $=$ TRISPHAT, $\Lambda$ - or $\Delta$-enantiomers) are known to shift the configurational equilibrium: $\mathrm{Fe}^{\mathrm{II}}(\mathrm{dm}-\mathrm{bpy})_{3}(\Lambda \text { - or } \Delta-\mathrm{TT})_{2}$ dissolved in the apolar $\mathrm{CHCl}_{3}$ forms contact ion pairs (CIP) with preferential homochiral association, e.g. $\Delta^{+}\left(\Delta^{-}\right)_{2}$, leading to very high diastereomeric excess $(d e>96 \%)$ over any heterochiral $\Lambda^{+}\left(\Delta^{-}\right)_{2}$ pairs present in the solution ${ }^{28,29}$. We compare the SCO dynamics of this sample (Fe $\left.{ }^{\mathrm{CIP}}\right)$ to two reference systems: 1) $\mathrm{Fe}^{\mathrm{II}}(\mathrm{dm}-\mathrm{bpy})_{3}(\Lambda \text { - or } \Delta \text {-TT })_{2}$ dissolved 
in the more polar $\mathrm{CH}_{2} \mathrm{Cl}_{2}\left(\mathrm{Fe}^{\mathrm{SIP}}\right)$, which forms a large proportion of solvent-separated ion pairs (SIP) with, consequently, a reduced diastereomeric interaction $(d e \approx 68 \%)^{30}$, and 2$) \mathrm{Fe}^{\mathrm{II}}(\mathrm{dm}-\mathrm{bpy})_{3}\left(\mathrm{PF}_{6}\right)_{2}$ in $\mathrm{CH}_{2} \mathrm{Cl}_{2}\left(\mathrm{Fe}^{\mathrm{RIP}}\right)$, which forms racemic ion-pairs (RIP) with the achiral $\mathrm{PF}_{6}$ anions. The sample synthesis is described in the Methods section.

It has been well established that photoexcitation of the HS state red-shifts the LC absorption band of the complex, providing an unambiguous spectroscopic signature of the HS state and therefore a probe of the SCO dynamics in the deep ultraviolet (UV) region $(270-350 \mathrm{~nm})^{7,8}$. Through a unique combination of ultrafast spectroscopic techniques in the deep-UV, we now exploit the polarization sensitivity of the LC transition dipoles in the HS state to directly resolve the structural mechanism of the back-SCO and its chiral control through the diastereomeric ion-pairing interaction. The associated optical setups and measurement conditions are described in the Methods section. We first establish a four-fold extension of the HS lifetime of $\mathrm{Fe}^{\mathrm{CIP}}$ compared to the racemic ion-pair $\mathrm{Fe}^{\mathrm{RIP}}$ using ultrafast broadband transient absorption (TA). Second, we use transient absorption anisotropy (TAA) to extract the conformational ensemble of the ligands, showing that the diastereomeric ion-pairing strongly narrows the width of their angular distribution around an ideal octahedral configuration. Finally, we employ a newly developed time-resolved circular dichroism (TRCD) technique with artifact-free broadband deep-UV detection ${ }^{31,32}$, delivering an unprecedented absolute sensitivity of $\pm 1 \cdot 10^{-5}$ OD. This breakthrough opens the avenue to spectrally resolve the excited state $\mathrm{CD}$ of photochemical systems in solution and to track its evolution with sub-picosecond resolution.

In this study we apply these novel capabilities to extract the excited state CD of the HS state and the structural dynamics it encodes. We identify the Ray-Dutt twist as a key reaction coordinate for the back-SCO, driving an asymmetric distortion of the $\mathrm{Fe}^{\mathrm{II}}$-centered coordination sphere on the picosecond time scale. Accounting for the TA and TAA experiments, we correlate the intrinsic rate of the distortion with the intersystem crossing rate to the ground state and the lability of the metal-ligand bonds. We thus find that the diastereomeric ion-pairing traps the HS state by cooling the vibrational population of the Ray-Dutt twist. Quite remarkably, this highlights the central importance of the associated torsional distortion in the back-SCO of $\mathrm{Fe}^{\mathrm{II}}$ complexes and implies that even in the prototypical $\left[\mathrm{Fe}^{\mathrm{II}}(\mathrm{bpy})_{3}\right]^{2+}$ more than a single active reaction coordinate is needed to fully describe its SCO mechanism. 


\section{Results}

\section{Chiral Fe" complexes}

The three investigated samples display qualitatively similar steady-state absorption spectra (for details see section S.1 in the Supplementary Information) with a strong band near $295 \mathrm{~nm}$ consisting of LC $\pi \pi^{*}$ transitions and a weaker MLCT band near $500 \mathrm{~nm}$ (see fig. S.1). Both bands display a double peak structure, which in the LC region is most pronounced for the racemic ion-pair $\mathrm{Fe}^{\mathrm{RIP}}$ and has been attributed to a vibrational progression localized on the ligands ${ }^{33,34}$. The deep-UV CD spectra of Fe $\mathrm{CIP}$ and $\mathrm{Fe}^{\mathrm{SIP}}$ (fig. S.2) display a characteristic bisignate structure resulting mostly from the excitonically coupled long-axis LC transitions, with the expected sign inversion for the $\Lambda$ and $\Delta$ configurations ${ }^{35,36}$. A spectral decomposition of the $\mathrm{CD}$ spectra (fig. S.3) shows that changing the solvent from $\mathrm{CHCl}_{3}$ to $\mathrm{CH}_{2} \mathrm{Cl}_{2}$ for $\mathrm{Fe}^{\mathrm{II}}(\mathrm{dm} \text {-bpy })_{3}(\Lambda \text { - or } \Delta \text {-TT })_{2}$ leads to a minor hypsochromic shift and a global reduction of the molar ellipticity with an enhanced suppression of the high-energy CD band centred near $285 \mathrm{~nm}$.

In the following we monitor different aspects of the back-SCO mechanism. We track the population dynamics of the HS state with TA and measure the linear dichroism of the photoexcited molecular ensemble with TAA. Note that TAA tracks the spatial distribution of LC transition dipoles in the HS state as a function of time, thus encoding the conformational ensemble of the ligands. Furthermore, we measure the chiral properties of excited HS state with TRCD, which is sensitive to changes in the excitonic coupling of the LC transition dipoles in the photoexcited complex. It is thus possible to directly resolve the structural changes that drive the relaxation dynamics of the HS state.

\section{Transient absorption}

Fig. 2a displays the TA spectrum of $\Lambda-\mathrm{Fe}^{\mathrm{CIP}}$ following MLCT excitation at $530 \mathrm{~nm}$ as a function of pump-probe delay. A pronounced ground state bleach (GSB) band centered at $295 \mathrm{~nm}$ takes the inverted shape of the steady-state absorption spectrum and overlaps with an excited state absorption (ESA) band centred at $315 \mathrm{~nm}$. This ESA band has previously been identified as the red-shifted LC absorption of the photoexcited complex in its HS state and has been well-established as a marker for its electronic and vibrational relaxation dynamics ${ }^{7,8}$. To this end, fig. 2 b displays the TA amplitude as a function of pump-probe delay for the HS absorption and the GSB bands near the spectral positions indicated by the 

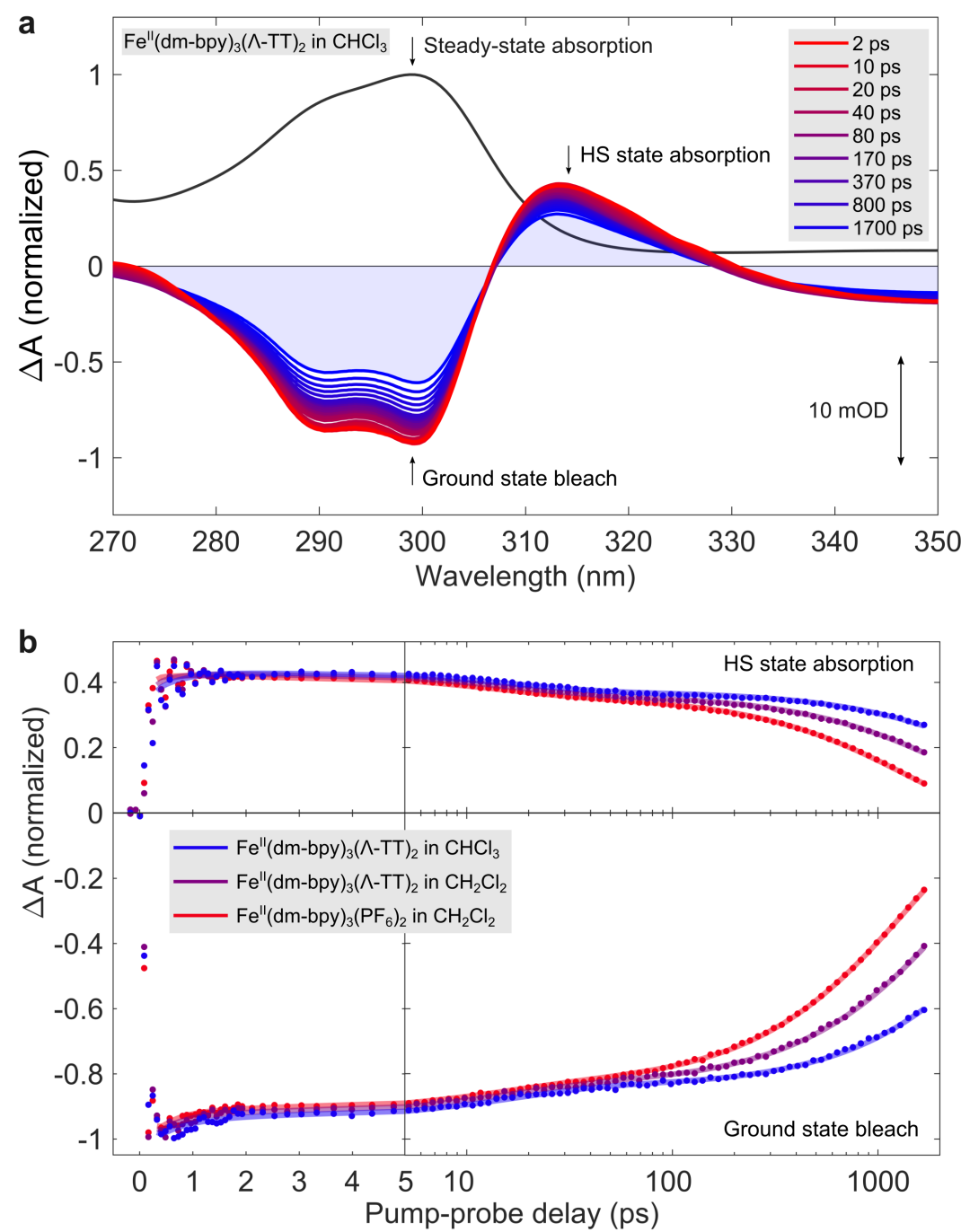

Figure 2. Transient absorption measurements show that diastereoselective ion-pairing extends the high-spin state lifetime of $\mathbf{F e}^{\mathrm{II}}(\mathbf{d m}-\mathbf{b p y})_{3}$. a, Deep ultraviolet steady-state and transient absorption of the ligand-centered transitions of the homochiral contact ion-pair $\mathrm{Fe}^{\mathrm{II}}(\mathrm{dm}-\mathrm{bpy})_{3}(\Lambda-\mathrm{TT})_{2}$ involving enantiopure counterions in $\mathrm{CHCl}_{3}$ after photoexcitation of its MLCT transition at $530 \mathrm{~nm}$. The probed spectral region simultaneously resolves the spectral fingerprints of the ground state bleach and the excited high-spin (HS) state. b, Multi-exponential HS state decay (top panel) and simultaneous ground state recovery dynamics (bottom panel) for ion-pairs with successively reduced diastereoselectivity: homochiral contact ion-pairs with maximum selectivity (blue), large proportion of separated ion-pairs in $\mathrm{CH}_{2} \mathrm{Cl}_{2}$ with reduced selectivity (purple), and racemic ion-pairs with achiral counterions in $\mathrm{CH}_{2} \mathrm{Cl}_{2}(\mathrm{red}$ ). To improve the visibility of the data, the vertical trace positions have been minimally adjusted and some data points at negative time delays have been omitted (the original traces are shown in the SI). A global analysis (solid lines) of the data (solid dots) determines an extension of the HS state lifetime to $5.0 \mathrm{~ns}$ in the contact ion-pair compared to $2.4 \mathrm{~ns}$ in the separated and $1.3 \mathrm{~ns}$ in the racemic ion-pair.

arrows in fig. $2 \mathrm{a}$ for $\mathrm{Fe}^{\mathrm{CIP}}, \mathrm{Fe}^{\mathrm{SIP}}$, and $\mathrm{Fe}^{\mathrm{RIP}}$. Note that the spectral positions were adapted to the band positions in each sample as indicated in fig. S.8 in the SI. We now observe a pronounced impact of the 
ion-pairing interaction on the TA kinetics: the HS state lives longest in $\mathrm{Fe}^{\mathrm{CIP}}$ with successively shorter lifetimes for $\mathrm{Fe}^{\mathrm{SIP}}$ and $\mathrm{Fe}{ }^{\mathrm{RIP}}$. We quantified the dynamics through a detailed spectro-kinetic analysis presented in section S.2, assuming a sequential decay model with three exponential components. For each sample, the HS state decay is dominated by the slowest component $\tau_{3}$, with $\tau_{3}^{\mathrm{CIP}}=4970 \pm 50 \mathrm{ps}$ for Fe ${ }^{\mathrm{CIP}}$, $\tau_{3}^{\mathrm{SIP}}=2370 \pm 20 \mathrm{ps}$ for $\mathrm{Fe}^{\mathrm{SIP}}$, and $\tau_{3}^{\mathrm{RIP}}=1330 \pm 10 \mathrm{ps}$ for $\mathrm{Fe}^{\mathrm{RIP}}$. The complete set of time constants is listed in table S.3, along with the decay constants obtained from the TAA and TRCD experiments.

\section{Transient anisotropy}

Fig. 3 displays the TAA measured for all three samples as a function of the pump-probe delay near the spectral positions corresponding to the TA kinetic traces presented in fig. $2 \mathrm{~b}$ (the sample-adapted positions are displayed in fig. S.12 in the SI). In the HS absorption region, TAA probes the relative orientation of LC transition dipoles in the HS state with respect to the initially excited MLCT transition. The initial TAA value $r_{0}$ at the earliest pump-probe delay displays a pronounced dependence on the ion-pairing interaction: the lowest value is obtained for $\mathrm{r}_{0}^{\mathrm{CIP}}=-0.96$, compared to $\mathrm{r}_{0}^{\mathrm{SIP}}=-0.87$ and $\mathrm{r}_{0}^{\mathrm{RIP}}=-0.66$. The TAA of the samples converges to zero on the $100 \mathrm{ps}$ time scale with increasingly faster kinetics going from $\mathrm{Fe}^{\mathrm{CIP}}$ to $\mathrm{Fe}^{\mathrm{SIP}}$ and $\mathrm{Fe}^{\mathrm{RIP}}$. Similar observations can be made for the TAA in the GSB region, however the differences in the initial TAA values are less pronounced and an additional decay component on the $10 \mathrm{ps}$ scale is clearly visible.

As TA bands typically contain contributions from different transition dipoles, the TAA across each TA band may vary strongly. In addition, zero crossings between GSB and ESA bands cause the TAA to diverge to infinity (see mathematical definition in Methods), which may distort the observed TAA kinetics. Fig. S.10 in the SI therefore displays the spectrally resolved TAA data for all three samples. Just as the TA spectrum, the TAA spectrum in the GSB region $<295 \mathrm{~nm}$ is composed of two bands with slightly different amplitudes. In the HS state absorption band no significant spectral variation can be observed, other than the divergence near its zero crossings. Both the presence of several transition dipoles and the dynamic changes of the TAA divergence near zero-crossings may be obscured in the TAA kinetics obtained from single-wavelength traces. Through suitably adapted global fits (for details see section S.3 in the SI), we found that the broadband data can be adequately fit with a minimum number of two exponential decays, in 

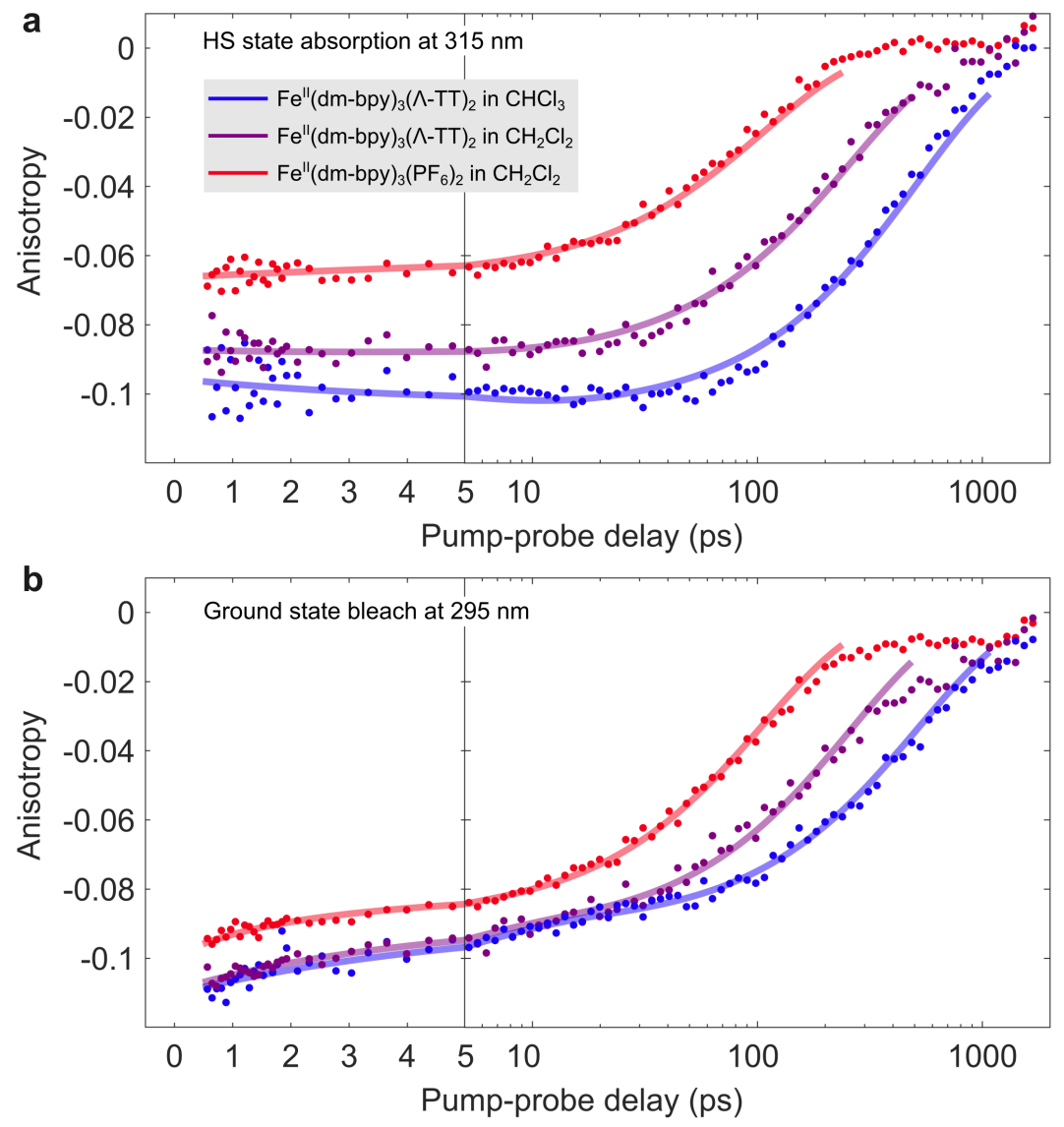

Figure 3. Transient absorption anisotropy experiments correlate the diastereoselective interaction with the conformational flexibility of $\mathrm{Fe}^{\mathrm{II}}(\mathbf{d m}-\mathrm{bpy})_{3}$ 's ligand system. a,b Transient absorption anisotropy after photoexcitation of the MLCT band at $530 \mathrm{~nm}$ as a function of the pump-probe delay in the HS state absorption (a) and ground state bleach region (b), as indicated in fig. 2a, for the contact (blue), the separated (purple), and the racemic ion-pair (red). Model calculations (see SI) correlate the measured initial anisotropy of the HS state $(<1 \mathrm{ps})$ with the width of the ligand system's conformational ensemble and find that the contact pair has a near-rigid octahedral ligand configuration with a three-times narrower conformational ensemble compared to the racemic pair. A global analysis of the multi-exponential anisotropy decays extracts picosecond structural dynamics in each sample's conformational ensemble and a characteristic rotational diffusion time as a global decay to zero. We observe that both processes are slowed down with increasing diastereoselective interaction.

line with the qualitative observations made above. With the time constants separated by more than an order of magnitude, both a sum and a sequential series of exponential functions can be employed to fit the data, resulting in identical time constants and exponential pre-factors for each sample. Fig. S.11 therefore only reports the results from the sequential model, whereas fig. S.12 shows the corresponding residuals. The exponential fits are included as solid lines in fig. 3 and display a very good correspondence with the data. The global analysis shows that the TAA dynamics across the chosen probe window can be described by 
the same two exponential processes, independent of the spectral position within the HS absorption or GSB band. For Fe ${ }^{\mathrm{CIP}}$, we obtained the time constants $\rho_{1}^{\mathrm{CIP}}=4.8 \pm 0.2$ ps and $\rho_{2}^{\mathrm{CIP}}=520 \pm 10$ ps. The shorter constant displays a minor amplitude across the probed spectral range and causes the TAA to decrease in the HS absorption band and to increase in the GSB band. The TAA then converges globally to zero with the longer time constant. Again, whilst qualitatively similar signatures are observed for $\mathrm{Fe}^{\mathrm{SIP}}$ and $\mathrm{Fe}^{\mathrm{RIP}}$, the ion-pairing interaction has a clear impact on their relative amplitudes and time scales: both processes become gradually faster for $\mathrm{Fe}^{\mathrm{SIP}}$ and $\mathrm{Fe}{ }^{\mathrm{RIP}}$, whereas the contribution of $\rho_{1}$ is successively decreased - as can be observed in the DAS and SAS in fig. S.11. For the time constants, we obtained $\rho_{1}^{\mathrm{SIP}}=3.6 \pm 0.2 \mathrm{ps}$ and $\rho_{2}^{\mathrm{SIP}}=260 \pm 10 \mathrm{ps}$ for $\mathrm{Fe}^{\mathrm{SIP}}$, and $\rho_{1}^{\mathrm{RIP}}=1.6 \pm 0.2 \mathrm{ps}$ and $\rho_{2}^{\mathrm{RIP}}=110 \pm 10 \mathrm{ps}$ for $\mathrm{Fe}^{\mathrm{RIP}}$.

\section{Time-resolved circular dichroism}

We conducted TRCD experiments on $\mathrm{Fe}^{\mathrm{CIP}}$ and $\mathrm{Fe}^{\mathrm{SIP}}$ in both their $\Lambda$ and $\Delta$ configurations and fig. 4a shows the time evolution of $\Lambda-\mathrm{Fe}^{\mathrm{CIP}}$ as an example, while the complete data sets are displayed in fig. S.17. The excellent signal-to-noise enables a detailed spectral analysis of the TRCD kinetics. Analogous to TA, the sign-inverted steady-state CD of the sample will appear in the TRCD spectrum as a GSB feature superimposed with the CD spectrum of the photoexcited HS state. However, whilst the decay of the GSB has to follow the ground state recovery dynamics observed in TA, the time evolution of the HS CD will also encode structural changes in the excited state. We thus identify GSB bands centered at $285 \mathrm{~nm}$ and $305 \mathrm{~nm}$ and a positive HS CD band near $315 \mathrm{~nm}$. In addition, the low initial amplitude of the GSB band at $285 \mathrm{~nm}$ compared to the steady-state $\mathrm{CD}$ and its weak decay dynamics suggest the presence of an overlapping HS CD band with a negative amplitude. In $\Lambda-\mathrm{Fe}^{\mathrm{SIP}}$ (fig. S.17c), the same spectral features are observed, yet with a faster global decay and a pronounced decay in the high-energy GSB band near $285 \mathrm{~nm}$. Importantly, we observe identical spectral dynamics with an inverted sign in the respective $\Delta$ configurations (fig. S.17b,d), demonstrating the consistency of the results.

We extracted the temporal evolution of the HS CD through a global fitting procedure (see Methods), showing that only two Gaussian HS CD bands with constrained fit parameters are needed to fit all data sets (for details see section S.4 in the SI). Fig. 4b displays the obtained time evolution of the HS CD for $\Lambda-\mathrm{Fe}^{\mathrm{CIP}}$ and $-\mathrm{Fe}^{\mathrm{SIP}}$, with fig. S.20 showing the associated residuals and the results obtained for the $\Delta$ 

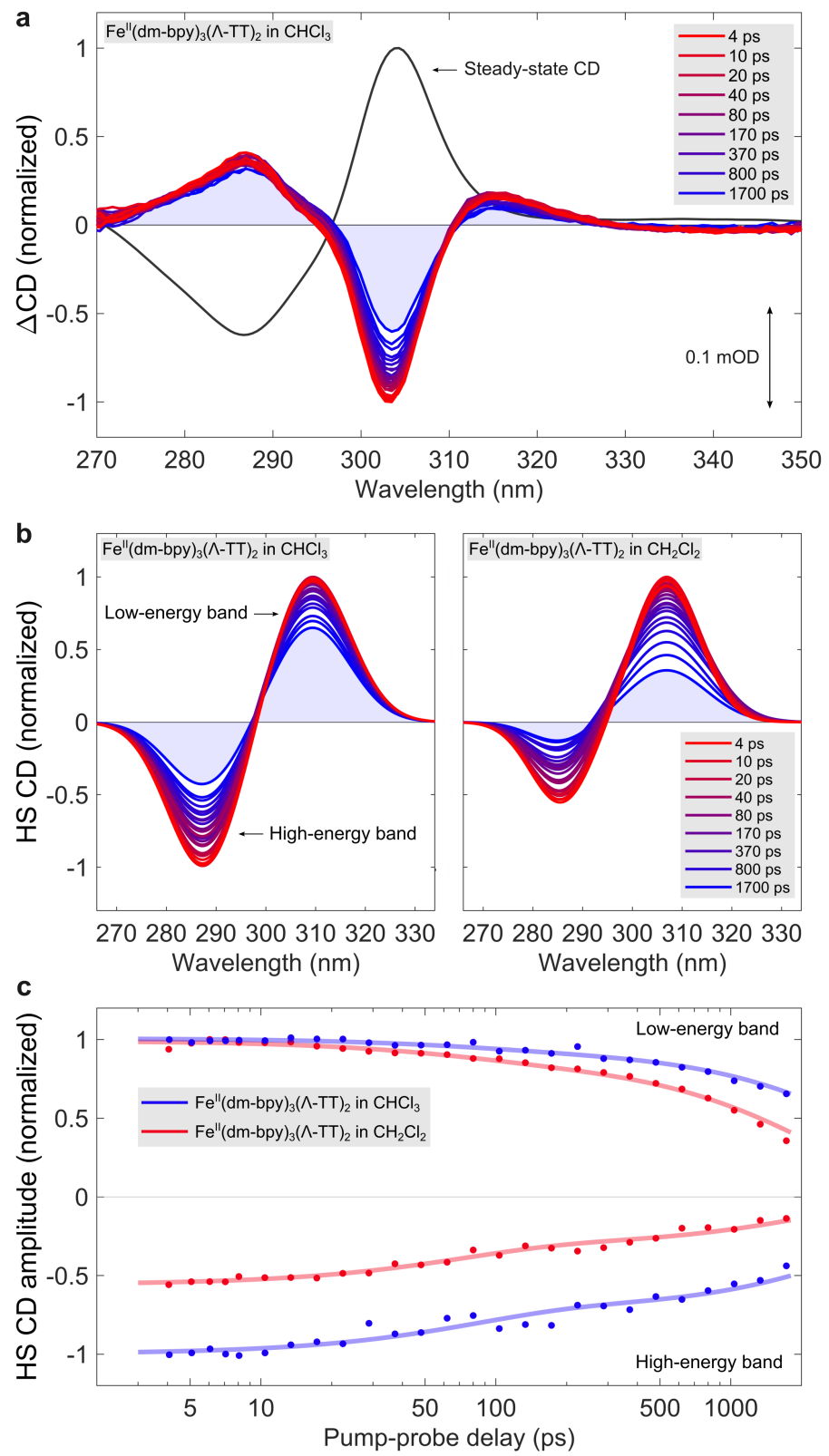

Figure 4. Time-resolved circular dichroism (CD) resolves an asymmetric decay of the high-spin state $\mathrm{CD}$ of $\mathrm{Fe}^{\mathrm{II}}(\mathbf{d m} \text {-bpy })_{3}$, indicating a symmetry-breaking structural distortion. a, Steady-state and time-resolved $\mathrm{CD}$ of the ligand-centered transitions of the homochiral contact ion-pair $\mathrm{Fe}^{\mathrm{II}}(\mathrm{dm} \text {-bpy })_{3}(\Lambda-\mathrm{TT})_{2}$ in $\mathrm{CHCl}_{3}$ after MLCT excitation at $530 \mathrm{~nm}$. Ground state bleach bands near 285 $\mathrm{nm}$ and $305 \mathrm{~nm}$ overlap with the CD spectrum of the excited HS state, with a positive band emerging near $315 \mathrm{~nm}$. b, We extracted the temporal evolution of the HS state's excitonic CD via a constrained global fit (for details see text and SI): in the contact ion-pair (left) an asymmetric decay of the HS CD bands strongly suppresses its high-energy band, whereas the separated ion-pair in $\mathrm{CH}_{2} \mathrm{Cl}_{2}$ (right) shows a similar, yet significantly enhanced effect. c, Normalized HS CD band amplitudes as a function of pump-probe delay. A double exponential fit (solid line) shows that the low-energy band mostly decays with the excited state population, whereas the high-energy band requires an additional 70 ps component, whose contribution is $\sim 28 \%$ in the contact and $\sim 43 \%$ in the separated ion-pair. 
configurations. In all cases, the bisignate HS CD bands follow the sign inversion of the steady-state CD, as expected from the chiral ligand framework. For Fe ${ }^{\mathrm{CIP}}$, the band amplitudes are mostly identical at early times, yet for increasing pump-probe delays the high-energy band decays more strongly, resulting in a significant reduction of the band ratio. For $\mathrm{Fe}^{\mathrm{SIP}}$, the reduced contact ion-pairing interaction enhances these effects: already at the earliest pump-probe delay, the high-energy band is much weaker than the low-energy band and its amplitude is suppressed more strongly with increasing pump-probe delay. In addition, a slight broadening of the low-energy band is observed. We thus note that the spectral evolution of the HS CD is dominated by an asymmetric decay of its bands, which is significantly more pronounced in $\mathrm{Fe}^{\mathrm{SIP}}$ compared to $\mathrm{Fe}^{\mathrm{CIP}}$.

To quantify the decay kinetics, fig. 4c plots the normalized band amplitudes as a function of pumpprobe delay. For both samples, the low-energy band decays nearly mono-exponentially, whereas an additional decay component on the 10 ps scale is clearly observed for the high-energy band. We thus simultaneously fitted the time evolution of the high- and low-energy band amplitudes with a sum of two exponential decays and accounted for the decay of the excited state population by setting one of the time constants to $\tau_{3}$, the longest decay constant obtained from the TA experiments. The fit curves are the solid lines in fig. 4c and are in excellent agreement with the data (see residuals in fig. S.22). The obtained fit parameters are displayed in table S.5 in the SI and reveal a decay component $\phi$ on the picosecond scale that is neither observed in the TA, nor in the TAA dynamics and takes the values $\phi^{\mathrm{CIP}}=70 \pm 10$ ps and $\phi^{\mathrm{SIP}}=80 \pm 10$ ps for $\mathrm{Fe}^{\mathrm{CIP}}$ and $\mathrm{Fe}^{\mathrm{SIP}}$, respectively. Whilst the time constants agree within their error ranges, their contribution to the decay of the high-energy HS CD band amplitude depends on the ion-pairing interaction: in $\mathrm{Fe}^{\mathrm{CIP}}$, the picosecond component contributes $\sim 28 \%$ to the total decay, whereas in $\mathrm{Fe}^{\mathrm{SIP}}$ this contribution is found to be $\sim 43 \%$. In the low-energy HS CD band, the contribution of the picosecond component accounts for $\sim 10 \%$ of the decay dynamics in both samples, implying that this band mostly decays as the excited state population.

\section{Discussion}

The diastereomeric interactions with either $\Lambda$ - or $\Delta$-TT counterions shift the configurational equilibrium of $\Lambda$ - and $\Delta$-Fe ${ }^{\mathrm{II}}\left(\mathrm{dm}^{\left.-\mathrm{bpy}_{3}\right)}\right.$ enantiomers towards the thermodynamically favorable homochiral $\Lambda$-Fe ${ }^{\mathrm{II}}(\mathrm{dm}-$ 
bpy $)_{3}(\Lambda-\mathrm{TT})_{2}$ or $\Delta-\mathrm{Fe}^{\mathrm{II}}(\mathrm{dm}-\mathrm{bpy})_{3}(\Delta-\mathrm{TT})_{2}$ pairs $^{37}$ : whilst $\mathrm{Fe}^{\mathrm{RIP}}$ is racemic, the homochiral ion-pairs $\Lambda, \Delta-\mathrm{Fe}^{\mathrm{CIP}}$ and $-\mathrm{Fe}^{\mathrm{SIP}}$ display a strong CD. Reddy et al. ${ }^{30}$ have determined that changing the solvent from $\mathrm{CHCl}_{3}$ to $\mathrm{CH}_{2} \mathrm{Cl}_{2}$ reduces the diastereomeric excess of homo- over heterochiral ion-pairs from $>96 \%$ to approximately $68 \%$. This is reflected in the reduction of the molar ellipticity of $\mathrm{Fe}^{\mathrm{SIP}}$ compared to $\mathrm{Fe}$, which is larger than the observed reduction in the associated molar extinction of the LC transitions. In the less polar $\mathrm{CHCl}_{3}$ (dielectric constant $\varepsilon=4.81$ ), the association with fluorinated TT counterions has further shown that $\mathrm{Fe}^{\mathrm{CIP}}$ is best described as a supramolecular complex with contact ion-pairing along the $\mathrm{C}_{3}$ axis of the $\mathrm{Fe}^{\mathrm{II}}$ complex ${ }^{29}$. However, due to a large degree of solvent-separated ion-pairing in the more polar $\mathrm{CH}_{2} \mathrm{Cl}_{2}(\varepsilon=8.93)$ of $\mathrm{Fe}^{\mathrm{SIP}}$, we cannot exclude the presence of structurally different supramolecular complexes, i.e. in the form of heterochiral ion-pairs with a less efficient pairing interaction along the $\mathrm{C}_{2}$ axes $^{38}$. However, in all reported experiments, $\mathrm{Fe}^{\mathrm{SIP}}$ displays the same qualitative spectral kinetics as $\mathrm{Fe}^{\mathrm{CIP}}$, suggesting equivalent excited state evolution without any evidence for the presence of additional, differently behaved species. We therefore assume that the higher proportion of heterochiral ion-pairs in $\mathrm{Fe}^{\mathrm{SIP}}$ displays the same electronic and mechanical properties as their homochiral counterparts, consistent with a simple inversion of the chiral structure of the $\mathrm{Fe}^{\mathrm{II}}$ complex within the ion-pair.

Through comparison with the well-characterized case of $\mathrm{Fe}^{\mathrm{II}}(\mathrm{bpy})_{3} \mathrm{Cl}_{2}$ in $\mathrm{H}_{2} \mathrm{O}$ (see details in section $\mathrm{S} .2$ in the SI), we directly assign the TA decay components $\tau_{2}$ and $\tau_{3}$ to the vibrational cooling and electronic decay of the HS state, respectively (the time constants obtained from all time-resolved experiments are listed in table S.3). Consequently, we can infer that the diastereomeric ion-pairing slows down both the vibrational cooling and the electronic decay of the HS state, whereas the latter effect is more strongly pronounced with a four-fold lengthening of the $\mathrm{HS}$ state lifetime when comparing $\mathrm{Fe}^{\mathrm{CIP}}$ to $\mathrm{Fe}^{\mathrm{RIP}}$. In this respect, the chosen series of investigated samples rules out simple solvation effects as the dominant cause: $\mathrm{Fe}^{\mathrm{RIP}}$ and $\mathrm{Fe}^{\mathrm{SIP}}$ have the same solvation environment, yet display strikingly different HS state dynamics. Whilst the much larger $\Lambda$-TT counterions may provide some shielding from the solvent, we note that this effect is minimized by the solvent-separated ion-pairing in $\mathrm{CH}_{2} \mathrm{Cl}_{2}$. This is consistent with recent findings by Miller and McCusker ${ }^{39}$ who found no significant difference in the HS state lifetime of $\left[\mathrm{Fe}^{\mathrm{II}}(\mathrm{bpy})_{3}\right]^{2+}$ paired with differently sized counterions in acetonitrile. The same study reports relatively small variations in the HS state lifetime with respect to the solvent polarity such that direct solvation 
differences are insufficient to explain the HS lifetimes obtained for $\mathrm{Fe}^{\mathrm{SIP}}$ and $\mathrm{Fe}^{\mathrm{CIP}}$. Instead, we conclude that the diastereomeric interaction with $\Lambda, \Delta$-TT controls the excited state lifetime through the manipulation of ligand system's configurational flexibility. This is a natural choice, since the enantiomerizing motion of the ligands is known to increase the spin-orbit coupling through the accompanying distortion of the metal-centered coordination sphere.

Concerning the vibrational cooling, we note that the rapid ${ }^{1} \mathrm{MLCT}-{ }^{3} \mathrm{MLCT}-{ }^{3} \mathrm{MC}-{ }^{5} \mathrm{~T}_{2} \mathrm{SCO}$ cascade leaves the HS state with approximately $1.3 \mathrm{eV}$ excess vibrational energy. Intramolecular vibrational energy redistribution (IVR) coupled to energy dissipation to the solvent then traps the HS state in a minimum energy conformation. It is well-established that the forward-SCO impulsively excites the totally symmetric breathing mode of the Fe-N bonds and local ligand bending and stretching modes ${ }^{7,8,13,17}$. The observed vibrational cooling times, with $\tau_{2}^{\mathrm{CIP}}>\tau_{2}^{\mathrm{SIP}}>\tau_{2}^{\mathrm{RIP}}$, therefore indicate that the diastereomeric ion-pairing decelerates the IVR process, most likely by reducing the anharmonic coupling terms through a reduced flexibility of the ligand system ${ }^{40}$.

The TAA experiments now enable a direct assessment of the ligand system's mechanical properties, as the measured spatial distribution of LC transition dipoles encodes the conformational ensemble of the ligands. To this end, photoexcitation of the isotropic sample in solution preferentially selects MLCT transition dipoles aligned parallel to the pump laser polarization. This creates an ensemble of excited molecules that is partially aligned within the laboratory frame of reference, following a $\cos ^{2} \alpha$ distribution, where $\alpha$ denotes the angle between the selected MLCT transition dipole and the pump polarization axis. TAA in the deep-UV then measures the relative orientation of the bleached ground state and excited HS state LC transition dipoles. Assuming an octahedral complex structure, a long-axis LC transition associated with the same ligand as the excited MLCT transition will result in the minimum anisotropy value of $r=-0.2$ due to its perpendicular arrangement, whereas the remaining two ligands each result in $r=-0.05^{41}$. Due to the back-electron transfer involved in forming the HS state, the three ligands become indistinguishable, such that the measured TAA in a rigid octahedral structure takes the average value $\langle\mathrm{r}\rangle=-0.1$. In order to correlate the experimental TAA with the conformational lability of the metal-ligand bonds, we introduced two spatial degrees of freedom in the calculation of the average anisotropy: a rotation angle $\theta$ of each ligand around the metal-ligand bond and a rotation around the metal-center in 
the ligand plane by an angle $\delta$. Note that $\theta=\delta=0^{\circ}$ corresponds to the octahedral configuration. In section S.3 we calculated $\langle\mathrm{r}\rangle$ as a function of the standard deviation $\sigma$ of a two-dimensional Gaussian distribution over both rotational degrees of freedom. The results are displayed in fig. S.13 and demonstrate that the value $\langle\mathrm{r}\rangle=-0.1$, obtained for a rigid ligand system with $\sigma=0^{\circ}$, is the minimum possible TAA value, whereas any broadening of the ligands' conformational distribution leads to an increase of $\langle\mathrm{r}\rangle$. The observed decrease in initial TAA values in the HS state absorption region, $\mathrm{r}_{0}^{\mathrm{RIP}}>\mathrm{r}_{0}^{\mathrm{SIP}}>\mathrm{r}_{0}^{\mathrm{CIP}}$, therefore shows that the diastereomeric ion-pairing interaction narrows the conformational distribution of the ligands around an octahedral structure of the complex, thereby counteracting the lability of the metal-ligand bonds. From the model calculations we obtained the standard deviations of the associated angular distributions $\sigma_{0}^{\mathrm{CIP}} \approx 7^{\circ}, \sigma_{0}^{\mathrm{SIP}} \approx 13^{\circ}$, and $\sigma_{0}^{\mathrm{RIP}} \approx 21^{\circ}$. We note that these results are based on a simplified model that does not consider possible steric interactions between the ligands at large angular displacements from the octahedral configuration. However, knowing that the ligands in $\mathrm{Fe}^{\mathrm{RIP}}$ can undergo large conformational changes upon its enantiomerization, we believe these contributions to be negligible over the obtained angular distributions. We further note that the experimentally obtained initial TAA values may be distorted by the TAA band divergences near $305 \mathrm{~nm}$ and $325 \mathrm{~nm}$ and by minor contributions from the TAA of the overlapping GSB band (for a spectral decomposition of the TA see fig. S.18). However, the broad width of the HS TAA band minimizes the impact of the divergences (see fig. S.10a,c,e) and the TAA values in the GSB band >290 nm closely match those in the HS state absorption region, such that their overlap becomes negligible.

To assess the time dependent evolution of the TAA we note that it must eventually converge to zero due to rotational diffusion of the photoselected molecular ensemble. The slowest time constant $\rho_{2}$ thus denotes the rotational diffusion time of each sample. As expected, $\mathrm{Fe}^{\mathrm{RIP}}$ displays the fastest rotational diffusion due to the smaller volume of the $\mathrm{PF}_{6}$ counterions and the lower viscosity of $\mathrm{CH}_{2} \mathrm{Cl}_{2}(\eta=0.41 \mathrm{cP})$ compared to $\mathrm{CHCl}_{3}(\eta=0.54 \mathrm{cP})$. However, the difference in solvent viscosity is too small to explain the difference between $\rho_{2}^{\mathrm{CIP}}$ and $\rho_{2}^{\mathrm{SIP}}$. As the global TAA decay to zero is well described by a single exponential in both cases, we apply the Stokes-Einstein-Debye diffusion equation for spherical particles in solution, such that $\rho_{2} \propto \eta \mathrm{V}$, where $\mathrm{V}$ is the rotational volume of the particle. We thus obtain $\mathrm{V}^{\mathrm{CIP}} \approx 1.5 \mathrm{~V}^{\mathrm{SIP}}$, which suggests that the contact ion-pair has a larger rotational volume than the separated ion-pair. We thus speculate 
that the larger interionic distance results in an increased global flexibility of the supramolecular complex, which may thus accelerate its diffusion in solution.

The second time constant $\rho_{1}$ is too fast to be associated with a rotational diffusion process. In the HS state absorption region it describes a minor, yet notable decrease in the measured TAA, which is most strongly pronounced in Fe ${ }^{\mathrm{CIP}}$. In view of the preceding discussion and the sub-10 ps time scale, we assign this process to a narrowing of the ligand system's conformational ensemble, due to the intramolecular vibrational energy redistribution in the HS state. In this respect, the value obtained for $\mathrm{Fe}^{\mathrm{RIP}}, \rho_{1}^{\mathrm{RIP}}=1.6 \pm 0.2 \mathrm{ps}$, agrees reasonably well with recent results from ultrafast electron diffraction experiments on $\mathrm{Fe}^{\mathrm{II}}(\mathrm{bpy})_{3}\left(\mathrm{PF}_{6}\right)_{2}$ single crystals, which determined a structural relaxation time of $2.4 \pm 0.4$ ps for the photoexcited HS state ${ }^{42}$. Surprisingly, $\rho_{1}$ describes an increase of the TAA in the GSB region with a much larger contribution to the dynamics compared to the HS state absorption region. We thus propose that the vibronic progression in the ground state absorption renders the GSB region more sensitive to the IVR process, albeit with a complex contribution to the obtained TAA value, as indicated by its variation across the GSB band.

We now turn to the TRCD experiments to determine the structural evolution of the HS state. For $\Lambda-\mathrm{Fe}^{\mathrm{CIP}}$, the sign inversion and equal band amplitudes of the HS CD at early pump-probe delays are consistent with an excitonic CD originating from the coupled long-axis LC transitions of the HS state, arranged in a near-rigid octahedral configuration. This assignment may seem at odds with the asymmetry of the corresponding steady-state CD bands, which are also known to be dominated by excitonically coupled LC transitions. In this case, however, the asymmetry is commonly assigned to overlapping electronic ground state transitions ${ }^{43,44}$, whereas for the HS state we found its absorption to be well described by a single absorption band (see fig. S.18a). Furthermore, its spectral position agrees very well with the zero-crossing of the bisignate $\mathrm{HS} \mathrm{CD}$, as expected from an excitonic coupling case.

In this view, the observed asymmetric decay of the HS CD is particularly striking, as it indicates a structural change that breaks the octahedral symmetry of the complex. Quite remarkably, this excludes the symmetric stretching mode of the metal-ligand bonds, which has commonly been assumed to be the single active reaction coordinate in the HS state decay of $\left[\mathrm{Fe}^{\mathrm{II}}(\mathrm{bpy})_{3}\right]^{2+4}$. This is supported by excitonic coupling and TDDFT calculations by Rudolph and co-workers ${ }^{44}$, which suggest that the steady-state LC CD band 
ratio is preserved for structural changes that maintain the three-fold rotation axis of the complex. These include equal changes in the three metal-ligand bond lengths, and equal out-of-plane ligand rotations around the bonds ( $\theta$ in the preceding discussion). We therefore propose that the asymmetric band decay requires a reaction coordinate that breaks the $C_{3}$ axis. In fact, it has previously been suggested that the resulting Jahn-Teller (JT) and pseudo Jahn-Teller (PJT) interactions may lead to a significant re-distribution of $\mathrm{CD}$ band intensities ${ }^{45}$. In the trigonal structure of $\left[\mathrm{Fe}^{\mathrm{II}}(\mathrm{dm}-\mathrm{bpy})_{3}\right]^{2+}$, the high-energy $\mathrm{CD}$ band is of non-degenerate $A_{2}$ symmetry and the low-energy band of doubly degenerate $E$ symmetry. Zgierski and Pawlikowski then calculated the impact of a vibronically coupled JT- and PJT-active mode of doubly degenerate $e$-symmetry on the steady-state $\mathrm{CD}^{46}$. The results show that the PJT interaction has a particularly strong impact on the CD band shapes with a pronounced suppression of the high-energy band compared to the low-energy band. On this basis, the asymmetric HS CD decay unambiguously identifies the racemizing Ray-Dutt twist with its $e$-symmetry ${ }^{47}$ as a reaction coordinate in the HS state decay of $\left[\mathrm{Fe}^{\mathrm{II}}(\mathrm{dm}-\mathrm{bpy})_{3}\right]^{2+}$. This assignment thus leads to a consistent mechanistic picture, where the diastereomeric ion-pairing controls the configurational equilibrium and lengthens the lifetime of the HS state of $\left[\mathrm{Fe}^{\mathrm{II}}(\mathrm{dm}-\mathrm{bpy})_{3}\right]^{2+}$ by counteracting the rotational lability of the metal-ligand bonds.

In this respect, the comparison with the HS CD evolution in $\Lambda-\mathrm{Fe}^{\mathrm{SIP}}$ offers further insights into the proposed reaction mechanism. At the earliest pump-probe delay, its HS CD is already asymmetric. This can now be explained by its broader angular conformational ensemble containing a larger fraction of distorted octahedral structures. We note that this is consistent with the spectral changes observed in the steady-state CD, where a similar suppression of the high-energy band was found for the solvent change from $\mathrm{Fe}^{\mathrm{CIP}}$ to $\mathrm{Fe}^{\mathrm{SIP}}$ (see fig. S.3 in the SI). From the time evolution of the HS CD band amplitudes we then extracted approximately the same time constant of 70 ps for both $\mathrm{Fe}^{\mathrm{CIP}}$ and $\mathrm{Fe}^{\mathrm{SIP}}$, however with a larger relative pre-exponential factor for $\mathrm{Fe}^{\mathrm{SIP}}$. Surprisingly, this suggests that the associated structural dynamics are largely equivalent for $\mathrm{Fe}^{\mathrm{CIP}}$ and $\mathrm{Fe}^{\mathrm{SIP}}$, whereas a stronger diastereomeric interaction mainly decreases the frequency of their occurrence.

In light of the obtained results, we now propose a refined reaction scheme for the back-SCO of trischelate $\mathrm{Fe}^{\mathrm{II}}$ complexes, illustrated in fig. 5. Upon photoexcitation of the LS ground state, a vibrationally excited HS state with elongated Fe-N bonds (HS*) is formed via an ultrafast forward-SCO. The HS* 


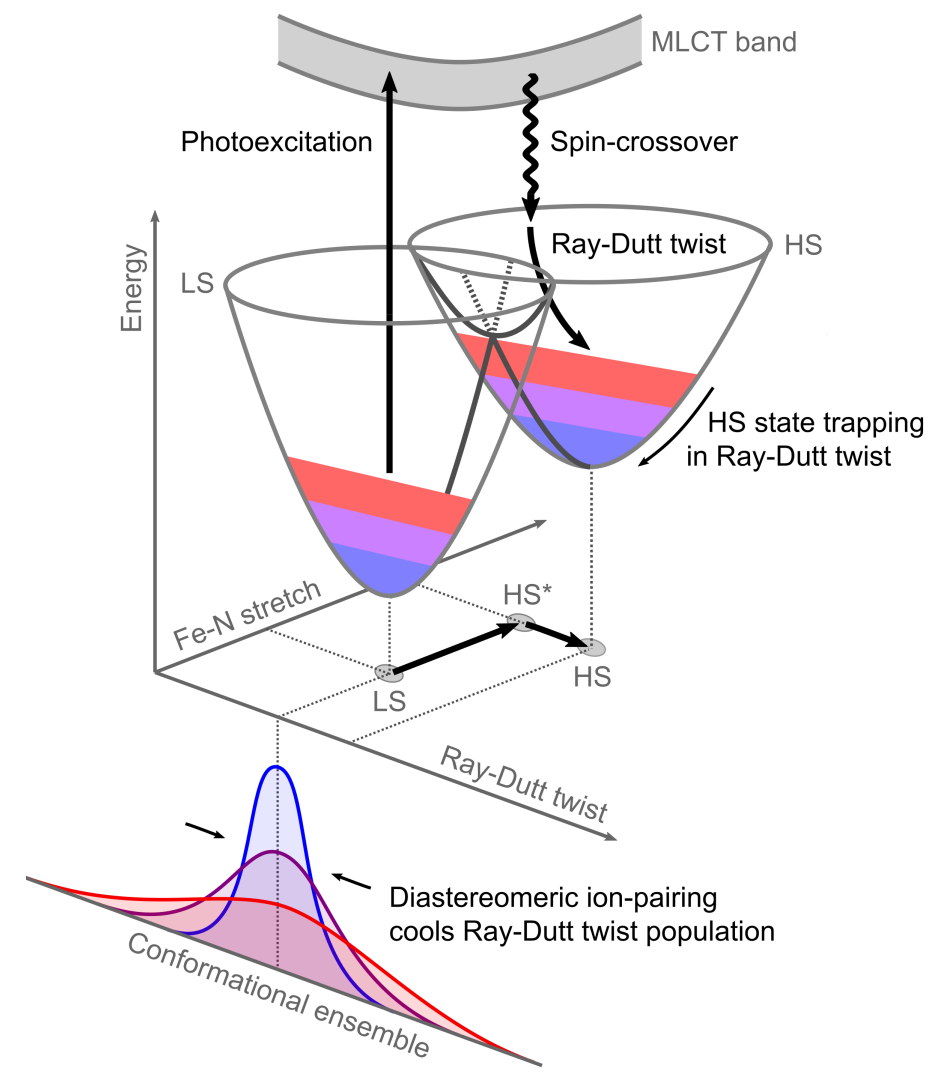

Figure 5. As the spin-relaxation of $\mathrm{Fe}^{\mathrm{II}}(\mathrm{dm}-\mathrm{bpy})_{3}$ is driven by the Ray-Dutt twist, the diasteromeric ion-pairing traps the HS state in its vibrational potential. The TRCD experiments show that $\mathrm{Fe}^{\mathrm{II}}(\mathrm{dm} \text {-bpy })_{3}$ is a two-mode system: photoexciation of its ground state (LS) creates a vibrationally excited HS state with expanded Fe-N bonds (HS*), which then evolves along the Ray-Dutt twisting coordinate to a distorted coordination configuration (HS), where an increased spin-orbit coupling facilitates the conversion back to the ground state. The diastereomeric ion-pairing narrows the conformational distribution of the ligands along the twisting coordinate, thus effectively cooling its vibrational population below the LS-HS barrier and extending the lifetime of the HS state.

state then evolves along the Ray-Dutt twisting coordinate towards its minimum energy configuration (HS)

in an entropy driven process with a 70 ps time constant. The resulting distortion of the $\mathrm{Fe}^{\mathrm{II}}$-centered coordination sphere enhances the spin-orbit coupling with the ground state and thus facilitates the decay of the HS state. Without any diastereomeric interaction, the lability of the Fe-N bonds implies a broad vibrational population of the Ray-Dutt twist in the LS ground state, as suggested by the broad angular conformational ensemble of the ligands. As illustrated in fig. 5, the forward-SCO transfers the broad vibrational population to the HS state. In the non-adiabatic multiphonon spin-relaxation mechanism developed by Buhks et al. ${ }^{14}$, this facilitates an efficient non-radiative coupling of the HS with the ground state. In this picture, the diastereomeric ion-pairing effectively cools the population of the Ray-Dutt twist, 
thus narrowing the angular conformational ensemble of the ligands. In direct analogy to the cryogenic cooling of vibrational modes in LIESST, the photoexcited HS state population then remains trapped in the potential of the Ray-Dutt twist. In this picture, the reduced diastereomeric interactions in $\mathrm{CH}_{2} \mathrm{Cl}_{2}$ increase the population of higher-order vibrational states without affecting the vibrational potential, thus increasing the rate of spin-relaxation, without any observable change in the time scale of the associated structural dynamics.

Along with the symmetric stretch mode of the Fe-N bonds, this establishes the symmetry-breaking Ray-Dutt twist as a key vibrational mode in the non-radiative coupling of the $\mathrm{HS}^{5} \mathrm{~T}_{2}$ and the $\mathrm{LS}{ }^{1} \mathrm{~A}_{1}$ state in octahedral Fe ${ }^{\mathrm{II}} \mathrm{SCO}$ complexes. $\left[\mathrm{Fe}^{\mathrm{II}}(\mathrm{bpy})_{3}\right]^{2+}$ thus becomes a two-mode system, much like the constrained $\left[\mathrm{Fe}^{\mathrm{II}}(\text { terpy })_{2}\right]^{2+}$, where anisotropic bond expansions also drive a Jahn-Teller active distortion of the coordination sphere in the HS state ${ }^{19}$. In this view, it may seem somewhat surprising that this mechanism has previously escaped direct detection in unconstrained tris-chelate Fe ${ }^{\mathrm{II}}$ complexes. However, already Purcell suggested that only a minor displacement along a torsional twisting coordinate would be required to induce a $\mathrm{SCO}^{24}$.

Almost 20 years after Hauser established $\left[\mathrm{Fe}^{\mathrm{II}}(\mathrm{bpy})_{3}\right]^{2+}$ as the archetypal SCO system following a single active reaction coordinate model $^{4}$, we can now conclude that this complex has become an exception to its own rule. However, the additional torsional twisting coordinate opens novel avenues for efficient spin-state trapping at RT of $\mathrm{Fe}^{\mathrm{II}}$-based $\mathrm{SCO}$ complexes by tuning the mechanical properties of their ligand system. Through the diastereomeric ion-pairing introduced in this study, we demonstrated that this can even be achieved without any chemical engineering of ligands. This novel diastereoselective supramolecular approach thus promises to become a particularly attractive strategy for trapping spin-states of chiral SCO complexes in both the liquid and the solid state, where it may directly complement existing ligand-field engineering approaches. Moreover, a similar strategy was recently pursued by Paulus et $a l$., who reduced the intersystem crossing rate from the MLCT manifold by suppressing the vibrational modes coupled to the HS state formation in a bridge-stabilized $\mathrm{Fe}^{\mathrm{II}}$ polypyridine complex ${ }^{48}$. Our results may thus also inform new strategies for kinetically stabilizing the photo-redox active MLCT and LMCT states of $\mathrm{Fe}^{\mathrm{II}}$ and $\mathrm{Fe}^{\mathrm{III}}$ complexes, respectively ${ }^{49,50}$. In a broader context, the presented results refine our understanding of SCO-induced structural dynamics at Fe sites, which play a fundamental role in the 
oxygen binding mechanism of heme-proteins and their function in the mammalian respiratory system, for example 51,52 .

Finally, this work enhances the scope of TRCD for resolving ultrafast structural dynamics of molecular systems in solution and demonstrates the unique advantages of this approach: due to the broad conformational ensemble determined for $\mathrm{Fe}^{\mathrm{RIP}}$, we speculate that the ensemble-averaged ligand twisting motions may currently be difficult to capture with established ultrafast X-Ray solution scattering techniques ${ }^{53}$. TRCD on the other hand is highly sensitive to the chiral properties of the excited state, such that even minor symmetry-breaking structural distortions can have a detectable impact on the excited state CD.

\section{Methods}

\section{Sample preparation}

Following literature procedures ${ }^{54,55},\left(\mathrm{NH}_{4}\right)_{2} \mathrm{Fe}\left(\mathrm{SO}_{4}\right)_{2} \cdot 6 \mathrm{H}_{2} \mathrm{O}(78 \mathrm{mg}, 0.2 \mathrm{mmol})$ and 4,4'-dimethyl-2,2'dipyridyl (111 mg, $0.6 \mathrm{mmol})$ were dissolved in $10 \mathrm{~mL}$ of an EtOH/water mixture (2/1) and refluxed for 30 minutes. The solution was cooled down and then added to a saturated solution of $\mathrm{KPF}_{6}$ in water. The precipitate was collected by filtration, washed with water and dried under reduced pressure. $145 \mathrm{mg}$ (yield $81 \%)$ of $\mathrm{Fe}^{\mathrm{II}}(\mathrm{dm} \text {-bpy })_{3}\left(\mathrm{PF}_{6}\right)_{2}$ were recovered. For the preparation of $\mathrm{Fe}^{\mathrm{II}}(\mathrm{dm}-\mathrm{bpy})_{3}(\Lambda, \Delta-\mathrm{TT})_{2}, 2$ equiv. of $\mathrm{P}(\mathrm{V})$ TRISPHAT anions $(\Lambda, \Delta-\mathrm{TT})^{56,57}$ were added to $\mathrm{Fe}^{\mathrm{II}}(\mathrm{dm}-\mathrm{bpy})_{3}\left(\mathrm{PF}_{6}\right)_{2}$ in a $\mathrm{CH}_{2} \mathrm{Cl}_{2} /$ acetone mixture (2:1) and the resulting complex was purified by column chromatography over silica gel $\left(\mathrm{CH}_{2} \mathrm{Cl}_{2}\right.$ as eluent). The observed diastereomeric ratio in $\mathrm{CDCl}_{3}$ was determined by $1 \mathrm{H}-\mathrm{NMR}$ spectroscopy and it was in agreement with literature data (diastereomeric ratio $d r>50: 1)^{28}$. For the ultrafast optical measurements, the sample was dissolved in spectroscopy grade $\mathrm{CHCl}_{3}$ and $\mathrm{CH}_{2} \mathrm{Cl}_{2}$ with a total volume of approximately $8 \mathrm{~mL}$ per experiment. Since $\mathrm{Fe}^{\mathrm{II}}(\mathrm{dm}-\mathrm{bpy})_{3}(\Lambda-\mathrm{TT})_{2}$ contained trace amounts of tributylamine $(<1 \mathrm{~mol} \%)$ from the preparation of the $\Lambda$-TT salt, $0.3 \mathrm{~mol} \%$ of tributylamine was added to the $\mathrm{Fe}^{\mathrm{II}}(\mathrm{dm}-\mathrm{bpy})_{3}(\Delta-\mathrm{TT})_{2}$ solutions, increasing the solubility of the compound. The sample concentration was adapted to achieve a maximum sample absorbance of approximately 0.7 OD near $300 \mathrm{~nm}$ for each measurement. For transient absorption (TA) and anisotropy (TAA) measurements, a $0.2 \mathrm{~mm}$ pathlength flow cell with ultrathin drilled windows (Starna) was used whereas for the time-resolved circular dichroism (TRCD) measurements, an equivalent $0.5 \mathrm{~mm}$ pathlength flow cell from low-strain quartz was employed. For all experiments, the 
sample was continuously circulated in a closed loop via a micro annular gear pump (HNP Mikrosysteme).

\section{Transient absorption and anisotropy in the deep-UV}

The laser setup for the ultrafast optical measurements has been described in detail elsewhere ${ }^{58,59}$. Briefly, a customized cryogenic Ti:Sapphire amplifier (KM Lasers) with an exceptional shot-to-shot stability of $0.1 \%$ root-mean-square, pumps a commercial non-collinear optical parametric amplifier (Light Conversion) at 20 $\mathrm{kHz}$. The broadband visible femtosecond pulses (520-720 nm) are split into a pump and probe arm via an ultrafast thin-film beamsplitter. The probe pulses are achromatically doubled in a thin $\mathrm{BBO}$ crystal $^{60}$ and cover $260-360 \mathrm{~nm}$. The pump pulses are spectrally filtered via several reflections off ultrafast multilayer mirrors, which limit the reflected bandwidth to approximately 520-575 $\mathrm{nm}$ at full-width-half-maximum (FWHM) (the spectrum is displayed in fig. S.1). The pump pulses are compressed via several reflections on a chirped mirror pair to compensate for any transmissive optics prior to the sample. The resulting pulse duration was determined with a commercial FROG system (Swamp Optics) to be approximately 15 fs.

For the transient absorption (TA) and anisotropy (TAA) experiments, the probe pulse was set to S-polarization with an achromatic half-wave plate before splitting the beam into a probe and a reference arm with a $1 \mathrm{~mm}$ thick reflective neutral density filter (0.3 OD). The probe was focussed into the sample with a $10 \mathrm{~cm}$ focal-length off-axis parabolic mirror, resulting in a focal spot diameter of approximately 70 $\mu \mathrm{m}\left(1 / \mathrm{e}^{2}\right)$. The probe polarization was cleaned with a high-quality Glan-Laser polarizer prior to detection with a fiber-coupled imaging spectrograph, equipped with two CMOS chips for recording the probe and reference simultaneously shot-to-shot. The pump polarization was cleaned via a Glan-Laser polarizer and switched between S- and P-polarization via a motorized achromatic half-wave plate. The pump was focussed via a $75 \mathrm{~cm}$ focal-length concave mirror to a spot diameter of approximately $270 \mu \mathrm{m}\left(1 / \mathrm{e}^{2}\right)$, where an energy of $0.8 \mu \mathrm{J}$ per pulse was used to photoexcite the sample. The resulting peak fluence

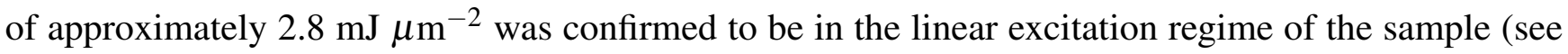
section S.2). At a given pump-probe delay t, 30,000 consecutive probe shots were acquired for each pump-polarization state, resulting in a TA spectrum for parallel pump and probe polarizations $\left(\mathrm{TA}^{\|}\right)$and a second for perpendicular polarizations $\left(\mathrm{TA}^{\perp}\right)$. The isotropic magic-angle TA and the TAA spectrum were then calculated as: 


$$
\mathrm{TA}(\lambda, \mathrm{t})=\frac{1}{3}\left(\mathrm{TA}^{\|}(\lambda, \mathrm{t})+2 \mathrm{TA}^{\perp}(\lambda, \mathrm{t})\right), \quad \operatorname{TAA}(\lambda, \mathrm{t})=\frac{1}{3}\left(\mathrm{TA}^{\|}(\lambda, \mathrm{t})-\mathrm{TA}^{\perp}(\lambda, \mathrm{t})\right) \times(\mathrm{TA}(\lambda, \mathrm{t}))^{-1}
$$

For the reported experiments, each pump-probe scan consisted of 104 pump-probe delays and was repeated 17 times for averaging. The instrument response function (IRF) of the measurements was determined in neat $\mathrm{CHCl}_{3}$ to be approximately 0.3 ps on average over the probed spectral region, with the data provided in the SI. To quantify the TA and TAA kinetics, we performed a global analysis for each data set with the OPTIMUS software package ${ }^{61}$, simultaneously fitting multi-exponential functions convoluted with the Gaussian IRF over the probed spectral window.

\section{Time-resolved circular dichroism in the deep-UV}

Circular dichroism (CD) denotes the differential absorption of left- and right-handed circularly polarized light and is sensitive to the spatial arrangement of excitonically coupled transition dipoles within a chiral molecular system ${ }^{62}$. However, its translation to the time domain for tracking the ultrafast structural dynamics of photochemical systems has remained a formidable challenge ${ }^{63}$, due to characteristically low signal levels in solution phase: the CD of common chiral molecules is $10^{-3}$ of their absorbance, such that time-resolved $\mathrm{CD}$ (TRCD) requires a sensitivity on the order of $10^{-5}$ to record photoinduced changes. On this level, polarization-sensitive optics easily distort broadband TRCD spectra, thus preventing their spectro-kinetic analysis and obscuring the relevant chiral dynamics. Our TRCD setup is described in detail in ref. ${ }^{31}$ and overcomes these issues through a broadband, artifact-free detection scheme with an unprecedented absolute sensitivity of $\pm 1 \cdot 10^{-5}$ OD. Briefly, the setup employs the same pump and probe pulses and detection system as the TA and TAA experiments. In addition, a photoelastic modulator is used to switch the probe pulse polarization shot-to-shot from left- to right-circularly polarized. Transmitted through a chiral sample, the intensity difference of consecutive probe pulses thus denotes the CD spectrum, which can be recorded for ground and excited state species in a pump-probe scheme analogous to TA spectroscopy. A TRCD spectrum is calculated by subtracting the ground state $\mathrm{CD}$ from the CD spectrum of the photoexcited sample. To acquire clean TRCD spectra, the suppression of polarization artifacts caused by static and pump-induced birefringence in the probe beam path is critical. Through the use of low- 
birefringence optics, probe-polarization scrambling prior to dispersive detection and careful calibration of the linear pump-polarization orientation, we measure an absolute sensitivity of $\pm 1 \cdot 10^{-5}$ OD and verified the absence of any artifact before each TRCD measurement through a baseline measurement with racemic $\left[\mathrm{Fe}^{\mathrm{II}}(\mathrm{bpy})_{3}\right]^{2+}$ (for the procedure and results see S.4). Due to the large amount of glass from the employed photoelastic modulator, lenses and polarizers, the probe pulses are stretched to approximately $500 \mathrm{fs}$. The IRF of the TRCD measurements was determined in neat $\mathrm{CHCl}_{3}$ to be approximately $0.5 \mathrm{ps}$ on average over the probed spectral region, with the data provided in the SI. The focal spot diameters of the probe and pump pulses were $70 \mu \mathrm{m}$ and $270 \mu \mathrm{m}\left(1 / \mathrm{e}^{2}\right)$, respectively and an energy of $1.0 \mu \mathrm{J}$ per pulse was used to photoexcite the sample. The corresponding peak fluence of $3.5 \mathrm{~mJ} \mu \mathrm{m}^{-2}$ was determined to be within the linear excitation regime (for details see section S.4). In order to reach the high signal-to-noise required for the presented spectral analysis, the probe pulse referencing was performed via a recently developed B-Matrix methodology ${ }^{64,65}$, which results in superior noise suppression compared to common ratiomeric pixel-by-pixel referencing schemes. For the reported experiments, 30,000 probe shots were acquired per pump-probe delay to calculate a TRCD spectrum, with 30 delay points forming a full pump-probe scan. For the the $\Lambda$-diastereoisomers, 800 scans were performed for averaging, taking about 18 hours in total. Due to the long data acquisition time, cross-checks with the $\Delta$-diastereoisomers were restricted to 10 time points and 600 scans. In order to extract the temporal evolution of the excited state $\mathrm{CD}$, we developed a global fitting procedure constrained by the expected GSB dynamics and a minimum number of excited state CD bands. A detailed discussion of this procedure is presented in section S.4 in the SI.

\section{Acknowledgements}

We thank J. Spekowius and J. Helbing (Zürich University) for adapting and sharing the B-Matrix referencing methodology and S. Grass (Geneva University) for the preparation of the enantiopure ammonium TRISPHAT salts. We also thank L. Müller and B. Bauer (EPFL) for assistance in the laboratory and X. Kong and C. Heinis (EPFL) for providing access to a steady-state CD spectrometer. Finally, we thank L. M. Lawson Daku (Geneva University), G. Pescitelli and F. Santoro (Pisa University) for helpful discussions. This work was supported by the Swiss NSF through the NCCR-MUST and by a fellowship within the Postdoc-Program of the German Academic Exchange Service (DAAD). 


\section{Author contributions statement}

$\mathrm{JL}$ and MO conceived the original idea. MO coordinated and carried out all aspects of the research (experiments, data analysis and interpretation), discussing them regularly with MC. FZ and JL contributed to sample preparation and manipulation. MO wrote the manuscript with contributions from all authors.

\section{Data availability statement}

The data that support the findings of this study are available from the corresponding author upon reasonable request.

\section{References}

1. Molnár, G., Rat, S., Salmon, L., Nicolazzi, W. \& Bousseksou, A. Spin Crossover Nanomaterials: From Fundamental Concepts to Devices. Adv. Mater. 30, 1703862, DOI: https://doi.org/10.1002/adma. 201703862 (2018).

2. Halcrow, M. A. (ed.) Spin-Crossover Materials: Properties and Applications (Wiley, Chichester, West Sussex, United Kingdom, 2013), 1st edition edn.

3. Hauser, A. Light-Induced Spin Crossover and the High-Spin $\rightarrow$ Low-Spin Relaxation. In Spin Crossover in Transition Metal Compounds II, vol. 234, 155-198, DOI: 10.1007/b95416 (Springer Berlin Heidelberg, Berlin, Heidelberg, 2004). Series Title: Topics in Current Chemistry.

4. Hauser, A., Enachescu, C., Daku, M. L., Vargas, A. \& Amstutz, N. Low-temperature lifetimes of metastable high-spin states in spin-crossover and in low-spin iron(II) compounds: The rule and exceptions to the rule. Coord. Chem. Rev. 250, 1642-1652, DOI: 10.1016/j.ccr.2005.12.006 (2006).

5. Gawelda, W. et al. Ultrafast Nonadiabatic Dynamics of [FeII(bpy)3]2+ in Solution. J. Am. Chem. Soc. 129, 8199-8206, DOI: 10.1021/ja070454x (2007).

6. Smeigh, A. L., Creelman, M., Mathies, R. A. \& McCusker, J. K. Femtosecond Time-Resolved Optical and Raman Spectroscopy of Photoinduced Spin Crossover: Temporal Resolution of Low-to-High Spin Optical Switching. J. Am. Chem. Soc. 130, 14105-14107, DOI: 10.1021/ja805949s (2008). Publisher: American Chemical Society.

7. Consani, C. et al. Vibrational Coherences and Relaxation in the High-Spin State of Aqueous [FeII(bpy)3]2+. Angewandte Chemie Int. Ed. 48, 7184-7187, DOI: 10.1002/anie.200902728 (2009).

8. Auböck, G. \& Chergui, M. Sub-50-fs photoinduced spin crossover in $\left[\mathrm{Fe}(\mathrm{bpy})_{3}\right]^{2+}$. Nat. Chem. 7 , 629-633, DOI: 10.1038/nchem.2305 (2015).

9. Gawelda, W. et al. Structural Determination of a Short-Lived Excited Iron(II) Complex by Picosecond X-Ray Absorption Spectroscopy. Phys. Rev. Lett. 98, 057401, DOI: 10.1103/PhysRevLett.98.057401 (2007). Publisher: American Physical Society. 
10. Bressler, C. et al. Femtosecond XANES Study of the Light-Induced Spin Crossover Dynamics in an Iron(II) Complex. Science 323, 489-492, DOI: 10.1126/science.1165733 (2009).

11. Huse, N. et al. Femtosecond Soft X-ray Spectroscopy of Solvated Transition-Metal Complexes: Deciphering the Interplay of Electronic and Structural Dynamics. The J. Phys. Chem. Lett. 2, 880-884, DOI: 10.1021/jz200168m (2011). Publisher: American Chemical Society.

12. Zhang, W. et al. Tracking excited-state charge and spin dynamics in iron coordination complexes. Nature 509, 345-348, DOI: 10.1038/nature13252 (2014). Number: 7500 Publisher: Nature Publishing Group.

13. Lemke, H. T. et al. Coherent structural trapping through wave packet dispersion during photoinduced spin state switching. Nat. Commun. 8, 15342, DOI: 10.1038/ncomms15342 (2017).

14. Buhks, E., Navon, G., Bixon, M. \& Jortner, J. Spin conversion processes in solutions. J. Am. Chem. Soc. 102, 2918-2923, DOI: 10.1021/ja00529a009 (1980).

15. Sutin, N. Nuclear, electronic, and frequency factors in electron transfer reactions. Accounts Chem. Res. 15, 275-282, DOI: 10.1021/ar00081a002 (1982).

16. Decurtins, S., Gütlich, P., Köhler, C. P., Spiering, H. \& Hauser, A. Light-induced excited spin state trapping in a transition-metal complex: The hexa-1-propyltetrazole-iron (II) tetrafluoroborate spin-crossover system. Chem. Phys. Lett. 105, 1-4, DOI: 10.1016/0009-2614(84)80403-0 (1984).

17. Cammarata, M. et al. Sequential Activation of Molecular Breathing and Bending during SpinCrossover Photoswitching Revealed by Femtosecond Optical and X-Ray Absorption Spectroscopy. Phys. Rev. Lett. 113, 227402, DOI: 10.1103/PhysRevLett.113.227402 (2014).

18. Nance, J., Bowman, D. N., Mukherjee, S., Kelley, C. T. \& Jakubikova, E. Insights into the SpinState Transitions in [Fe(tpy)2]2+: Importance of the Terpyridine Rocking Motion. Inorg. Chem. 54, 11259-11268, DOI: 10.1021/acs.inorgchem.5b01747 (2015). Publisher: American Chemical Society.

19. Zhang, W. \& Gaffney, K. J. Mechanistic Studies of Photoinduced Spin Crossover and Electron Transfer in Inorganic Complexes. Accounts Chem. Res. 48, 1140-1148, DOI: 10.1021/ar500407p (2015). Publisher: American Chemical Society.

20. Stock, P. et al. Molecular Spin Crossover in Slow Motion: Light-Induced Spin-State Transitions in Trigonal Prismatic Iron(II) Complexes. Inorg. Chem. 55, 5254-5265, DOI: 10.1021/acs.inorgchem. 6 b00238 (2016). Publisher: American Chemical Society.

21. Marchivie, M., Guionneau, P., Létard, J.-F. \& Chasseau, D. Photo-induced spin-transition: the role of the iron(II) environment distortion. Acta Crystallogr. Sect. B Struct. Sci. 61, 25-28, DOI: 10.1107/S0108768104029751 (2005).

22. Halcrow, M. Structure:function relationships in molecular spin-crossover complexes. Chem. Soc. Rev. 40, 4119-4142, DOI: 10.1039/C1CS15046D (2011). Publisher: Royal Society of Chemistry.

23. Purcell, K. F. \& Zapata, J. P. Magnetic isomers. cisBis(cyanotriphenylborato)bisphenanthrolineiron(II). J. Chem. Soc. Chem. Commun. 497-499, DOI: 10.1039/C39780000497 (1978). Publisher: The Royal Society of Chemistry.

24. Purcell, K. F. Pseudorotational intersystem crossing in d6 complexes. J. Am. Chem. Soc. 101, 5147-5152, DOI: 10.1021/ja00512a005 (1979). Publisher: American Chemical Society. 
25. Vanquickenborne, L. G. \& Pierloot, K. Role of spin change in the stereomobile reactions of strongfield d6 transition-metal complexes. Inorg. Chem. 20, 3673-3677, DOI: 10.1021/ic50225a018 (1981). Publisher: American Chemical Society.

26. McCusker, J. K., Rheingold, A. L. \& Hendrickson, D. N. Variable-Temperature Studies of LaserInitiated 5T2 $\rightarrow$ 1A1 Intersystem Crossing in Spin-Crossover Complexes: Empirical Correlations between Activation Parameters and Ligand Structure in a Series of Polypyridyl Ferrous Complexes. Inorg. Chem. 35, 2100-2112, DOI: 10.1021/ic9507880 (1996). Publisher: American Chemical Society.

27. Ashley, D. C. \& Jakubikova, E. Ray-Dutt and Bailar Twists in Fe(II)-Tris(2,2'-bipyridine): Spin States, Sterics, and Fe-N Bond Strengths. Inorg. Chem. 57, 5585-5596, DOI: 10.1021/acs.inorgchem.8b00560 (2018). Publisher: American Chemical Society.

28. Lacour, J., Jodry, J. J., Ginglinger, C. \& Torche-Haldimann, S. Diastereoselective Ion Pairing of TRISPHAT Anions and Tris(4,4'-dimethyl-2,2'-bipyridine)iron(II). Angewandte Chemie Int. Ed. 37, 2379-2380, DOI: 10.1002/(SICI)1521-3773(19980918)37:17<2379::AID-ANIE2379>3.0.CO;2-C (1998).

29. Frantz, R., Pinto, A., Constant, S., Bernardinelli, G. \& Lacour, J. Fluorinated TRISPHAT Anions: Spectroscopic Probes for Detailed Asymmetric Ion Pairing Studies. Angewandte Chemie Int. Ed. 44, 5060-5064, DOI: 10.1002/anie.200500862 (2005).

30. Reddy, G. M., Ballesteros-Garrido, R., Lacour, J. \& Caldarelli, S. Determination of Labile Chiral Supramolecular Ion Pairs by Chromatographic NMR Spectroscopy. Angewandte Chemie Int. Ed. 52, 3255-3258, DOI: 10.1002/anie.201209616 (2013).

31. Oppermann, M. et al. Ultrafast broadband circular dichroism in the deep ultraviolet. Optica 6, 56-60, DOI: 10.1364/OPTICA.6.000056 (2019).

32. Oppermann, M. et al. Broad-Band Ultraviolet CD Spectroscopy of Ultrafast Peptide Backbone Conformational Dynamics. The J. Phys. Chem. Lett. 10, 2700-2705, DOI: 10.1021/acs.jpclett.9b01253 (2019).

33. Mason, S. F., Peart, B. J. \& Waddell, R. E. Optical rotatory power of co-ordination compounds. Part XVI. Intermediate exciton coupling in the circular dichroism of trisbipyridyl complexes. J. Chem. Soc. Dalton Transactions 0, 944-949, DOI: 10.1039/DT9730000944 (1973). Publisher: Royal Society of Chemistry.

34. Castellucci, E., Salvi, P. R. \& Foggi, P. Two-photon excitation spectra of the lowest electronic states of 2,2'-bipyridine. Chem. Phys. 66, 281-291, DOI: 10.1016/0301-0104(82)88027-0 (1982).

35. Mason, S. F. The electronic spectra and optical activity of phenanthroline and dipyridyl metal complexes. Inorganica Chimica Acta Rev. 2, 89-109, DOI: 10.1016/0073-8085(68)80016-6 (1968).

36. Bosnich, B. Application of exciton theory to the determination of the absolute configurations of inorganic complexes. Accounts Chem. Res. 2, 266-273, DOI: 10.1021/ar50021a002 (1969).

37. Reist, M., Testa, B., Carrupt, P.-A., Jung, M. \& Schurig, V. Racemization, enantiomerization, diastereomerization, and epimerization: Their meaning and pharmacological significance. Chirality 7 , 396-400, DOI: 10.1002/chir.530070603 (1995).

38. Jodry, J. J., Frantz, R. \& Lacour, J. Supramolecular Stereocontrol of Octahedral Metal-Centered Chirality. Ligand Modulation. Inorg. Chem. 43, 3329-3331, DOI: 10.1021/ic049661d (2004). 
39. Miller, J. N. \& McCusker, J. K. Outer-sphere effects on ligand-field excited-state dynamics: solvent dependence of high-spin to low-spin conversion in [Fe(bpy) 3 ] 2+. Chem. Sci. 11, 5191-5204, DOI: 10.1039/D0SC01506G (2020). Publisher: Royal Society of Chemistry.

40. Bethardy, G., Wang, X. \& Perry, D. S. The role of molecular flexibility in accelerating intramolecular vibrational relaxation. Can. J. Chem. 72, 652-659, DOI: 10.1139/v94-090 (1994).

41. Wallin, S., Davidsson, J., Modin, J. \& Hammarström, L. Femtosecond Transient Absorption Anisotropy Study on $[\mathrm{Ru}(\mathrm{bpy}) 3] 2+$ and $[\mathrm{Ru}(\mathrm{bpy})(\mathrm{py}) 4] 2+$. Ultrafast Interligand Randomization of the MLCT State. The J. Phys. Chem. A 109, 4697-4704, DOI: 10.1021/jp0509212 (2005).

42. Jiang, Y. et al. Direct observation of nuclear reorganization driven by ultrafast spin transitions. Nat. Commun. 11, 1-8, DOI: 10.1038/s41467-020-15187-y (2020). Number: 1 Publisher: Nature Publishing Group.

43. Fan, J., Autschbach, J. \& Ziegler, T. Electronic Structure and Circular Dichroism of Tris(bipyridyl) Metal Complexes within Density Functional Theory. Inorg. Chem. 49, 1355-1362, DOI: 10.1021/ ic9011586 (2010). Publisher: American Chemical Society.

44. Rudolph, M. \& Autschbach, J. Calculation of Optical Rotatory Dispersion and Electronic Circular Dichroism for Tris-bidentate Groups 8 and 9 Metal Complexes, With Emphasis on Exciton Coupling. The J. Phys. Chem. A 115, 2635-2649, DOI: 10.1021/jp111484z (2011). Publisher: American Chemical Society.

45. Richardson, F., Caliga, D., Hilmes, G. \& Jenkins, J. Vibronic effects in the chiroptical spectra of dissymmetric trigonal systems. Mol. Phys. 30, 257-280, DOI: 10.1080/00268977500101931 (1975).

46. Zgierski, M. Z. \& Pawlikowski, M. Jahn-Teller, pseudo Jahn-Teller coupling, and circular dichroism spectra of (E+A) e systems. The J. Chem. Phys. 70, 3444-3452, DOI: 10.1063/1.437879 (1979). Publisher: American Institute of Physics.

47. Rodger, A. Template symmetry restrictions on reaction mechanisms. Inorganica Chimica Acta 185, 193-200, DOI: 10.1016/S0020-1693(00)85444-4 (1991).

48. Paulus, B. C., Adelman, S. L., Jamula, L. L. \& McCusker, J. K. Leveraging excited-state coherence for synthetic control of ultrafast dynamics. Nature 582, 214-218, DOI: 10.1038/s41586-020-2353-2 (2020). Number: 7811 Publisher: Nature Publishing Group.

49. McCusker, J. K. Electronic structure in the transition metal block and its implications for light harvesting. Science 363, 484-488, DOI: 10.1126/science.aav9104 (2019). Publisher: American Association for the Advancement of Science Section: Review.

50. Wenger, O. S. Is Iron the New Ruthenium? Chem. - A Eur. J. 25, 6043-6052, DOI: https://doi.org/10. 1002/chem.201806148 (2019).

51. Kepp, K. P. Heme: From quantum spin crossover to oxygen manager of life. Coord. Chem. Rev. 344, 363-374, DOI: 10.1016/j.ccr.2016.08.008 (2017).

52. Kinschel, D. et al. Femtosecond X-ray emission study of the spin cross-over dynamics in haem proteins. Nat. Commun. 11, 4145, DOI: 10.1038/s41467-020-17923-w (2020).

53. Ki, H., Oang, K. Y., Kim, J. \& Ihee, H. Ultrafast X-Ray Crystallography and Liquidography. Annu. Rev. Phys. Chem. 68, 473-497, DOI: 10.1146/annurev-physchem-052516-050851 (2017). Publisher: Annual Reviews. 
54. Pomeranc, D., Heitz, V., Chambron, J.-C. \& Sauvage, J.-P. Octahedral Fe(II) and Ru(II) Complexes Based on a New Bis 1,10-Phenanthroline Ligand That Imposes a Well Defined Axis. J. Am. Chem. Soc. 123, 12215-12221, DOI: 10.1021/ja011250y (2001). Publisher: American Chemical Society.

55. Cazzanti, S., Caramori, S., Argazzi, R., Elliott, C. M. \& Bignozzi, C. A. Efficient Non-corrosive Electron-Transfer Mediator Mixtures for Dye-Sensitized Solar Cells. J. Am. Chem. Soc. 128, 99969997, DOI: 10.1021/ja062087f (2006). Publisher: American Chemical Society.

56. Lacour, J., Ginglinger, C., Grivet, C. \& Bernardinelli, G. Synthesis and Resolution of the Configurationally Stable Tris(tetrachlorobenzenediolato)phosphate(V) Ion. Angewandte Chemie Int. Ed. Engl. 36, 608-610, DOI: https://doi.org/10.1002/anie.199706081 (1997).

57. Favarger, F., Goujon-Ginglinger, C., Monchaud, D. \& Lacour, J. Large-Scale Synthesis and Resolution of TRISPHAT [Tris(tetrachlorobenzenediolato) Phosphate(V)] Anion. The J. Org. Chem. 69, 85218524, DOI: 10.1021/jo048641q (2004). Publisher: American Chemical Society.

58. Auböck, G. et al. Femtosecond pump/supercontinuum-probe setup with $20 \mathrm{kHz}$ repetition rate. Rev. Sci. Instruments 83, 093105, DOI: 10.1063/1.4750978 (2012).

59. Auböck, G., Consani, C., Mourik, F. v. \& Chergui, M. Ultrabroadband femtosecond two-dimensional ultraviolet transient absorption. Opt. Lett. 37, 2337-2339, DOI: 10.1364/OL.37.002337 (2012).

60. Baum, P., Lochbrunner, S. \& Riedle, E. Tunable sub-10-fs ultraviolet pulses generated by achromatic frequency doubling. Opt. Lett. 29, 1686-1688, DOI: 10.1364/OL.29.001686 (2004).

61. Slavov, C., Hartmann, H. \& Wachtveitl, J. Implementation and Evaluation of Data Analysis Strategies for Time-Resolved Optical Spectroscopy. Anal. Chem. 87, 2328-2336, DOI: 10.1021/ac504348h (2015).

62. Berova, N., Polavarapu, P. L., Nakanishi, K. \& Woody, R. W. Comprehensive Chiroptical Spectroscopy: Instrumentation, Methodologies, and Theoretical Simulations (Wiley, Hoboken, NJ, 2012), volume 1 edition edn.

63. Meyer-Ilse, J., Akimov, D. \& Dietzek, B. Recent advances in ultrafast time-resolved chirality measurements: perspective and outlook: Ultrafast transient molecular chirality. Laser \& Photonics Rev. 7, 495-505, DOI: 10.1002/lpor.201200065 (2013).

64. Feng, Y., Vinogradov, I. \& Ge, N.-H. General noise suppression scheme with reference detection in heterodyne nonlinear spectroscopy. Opt. Express 25, 26262-26279, DOI: 10.1364/OE.25.026262 (2017). Publisher: Optical Society of America.

65. Feng, Y., Vinogradov, I. \& Ge, N.-H. Optimized noise reduction scheme for heterodyne spectroscopy using array detectors. Opt. Express 27, 20323-20346, DOI: 10.1364/OE.27.020323 (2019). Publisher: Optical Society of America. 


\section{Supporting Information}

\section{Contents}

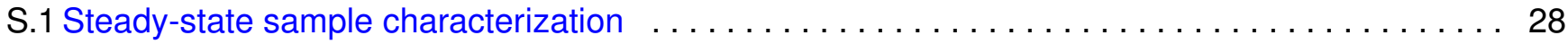

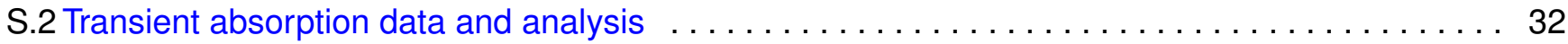

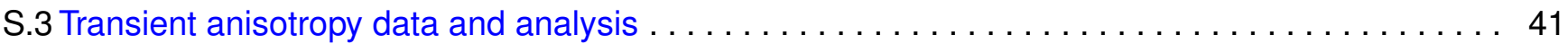

S.4 Time-resolved circular dichroism data and analysis $\ldots \ldots \ldots \ldots \ldots \ldots \ldots \ldots \ldots \ldots \ldots$

$\begin{array}{ll}\text { References Supporting Information } & 61\end{array}$

\section{S.1 Steady-state sample characterization}

Three samples were investigated: 1) $\mathrm{Fe}^{\mathrm{II}}(\mathrm{dm}-\mathrm{bpy})_{3}(\Lambda, \Delta-\mathrm{TT})_{2}$ dissolved in $\left.\mathrm{CHCl}_{3}\left(\Lambda, \Delta-\mathrm{Fe}^{\mathrm{CIP}}\right), 2\right) \mathrm{Fe}^{\mathrm{II}}(\mathrm{dm}-$ bpy $)_{3}(\Lambda, \Delta-\mathrm{TT})_{2}$ dissolved in $\mathrm{CH}_{2} \mathrm{Cl}_{2}\left(\Lambda, \Delta-\mathrm{Fe}^{\mathrm{SIP}}\right)$, and 3) $\mathrm{Fe}^{\mathrm{II}}(\mathrm{dm}-\mathrm{bpy})_{3}\left(\mathrm{PF}_{6}\right)_{2}$ dissolved in $\mathrm{CH}_{2} \mathrm{Cl}_{2}$ $\left(\mathrm{Fe}^{\mathrm{RIP}}\right)$. Their steady-state absorption and CD spectra were obtained with a JASCO J-1100 CD spectrometer using a $1 \mathrm{~mm}$ pathlength cuvette. The maximum absorption of the samples in the ultraviolet (UV) region was kept between 0.8 and 1.5 OD in order to achieve a good signal-to-noise whilst avoiding saturation of the detector. All spectra were baseline-corrected via a solvent blank. Fig. S.1 displays the absorption spectra of all three samples normalized to their respective absorption maxima in the plotted spectral region. The left panel displays the absorption in the deep-UV region, which is dominated by the ligand-centred (LC) $\pi \pi^{*}$ transitions of the complexes. $\mathrm{Fe}^{\mathrm{CIP}}$ and $\mathrm{Fe}^{\mathrm{SIP}}$ display nearly identical band shapes with a maximum near $300 \mathrm{~nm}$ and a shoulder near $290 \mathrm{~nm}$. Changing the solvent from $\mathrm{CHCl}_{3}$ to $\mathrm{CH}_{2} \mathrm{Cl}_{2}$ results in a minor hypsochromic shift of about $1 \mathrm{~nm}$. In direct comparison, $\mathrm{Fe}^{\mathrm{RIP}}$ displays a more pronounced hypsochromic shift, a reduced shoulder, a strongly enhanced absorption $<270 \mathrm{~nm}$, and an additional broad shoulder at $320 \mathrm{~nm}$. We attribute the latter to the absorption of the $\mathrm{PF}_{6}$ counterions. The right panel displays the absorption in the visible region and shows the metal-to-ligand charge-transfer (MLCT) band. The amplitude of this band is about 10\% of the LC band and shows a similar spectral shape. It has been suggested that the double peak structure in the LC band stems from a vibrational progression localized on the ligands si1, si2. For the MLCT band, similar interpretations have been proposed, pointing to a skeletal mode at $1607 \mathrm{~cm}^{-1 \mathrm{si} 3}$. However, Decurtins et al. have suggested that its double band structure 
results from two partially overlapping electronic transitions ${ }^{\mathrm{si} 4}$.

Fig. S.2 displays the steady-state $\mathrm{CD}$ spectra of $\mathrm{Fe}^{\mathrm{CIP}}$ and $\mathrm{Fe}{ }^{\mathrm{SIP}}$. Both display near identical spectral shapes, dominated by two bands of opposite signs: 1) a high-energy band near $285 \mathrm{~nm}$, and 2) a low-energy band near $303 \mathrm{~nm}$. This bisignate spectral structure is a typical signature of excitonically coupled electronic transitions and for tris-bipyridyl complexes it has been established that the CD in this region is dominated by the long-axis transitions of the bpy ligands ${ }^{\mathrm{si} 5}$, si6 . Due to the $\mathrm{D}_{3}$ symmetry of the ligand system, the high-energy band is of $A_{2}$ symmetry, whilst the low-energy band is degenerate with $E$-symmetry. In this environment, the excitonic transitions from the coupled short-axis transitions are not optically active. The observed absence of any vibronic structure in the CD spectra is a known effect in tris-bipyridyl complexes, where the exciton splitting energy is typically much larger than the energy of the vibronically coupled mode (see for example the discussion in ref. ${ }^{\text {sil }}$ ).

In order to assess the impact of the solvent change on the diastereomeric ion pairs, we analyze their CD spectra in more detail. The rotational strengths $\mathrm{R}_{A_{2}, E}$ of the two main bands are strongly reduced, such that for the high-energy band $\mathrm{R}_{A_{2}}^{\mathrm{SIP}} / \mathrm{R}_{A_{2}}^{\mathrm{CIP}} \approx 0.52$ and for the low-energy band $\mathrm{R}_{E}^{\mathrm{SIP}} / \mathrm{R}_{E}^{\mathrm{CIP}} \approx 0.64$. In both cases, the reduction in amplitude is larger than the reduction in the maximum absorption coefficient $\varepsilon$ (see fig. S.3a), which gives $\varepsilon^{\mathrm{SIP}} / \varepsilon^{\mathrm{CIP}} \approx 0.75$. This is to be expected, since the diastereomeric ratio decreases when the solvent polarity is increased. However, this does not rationalize the change in band ratio, which is directly visible when the $\mathrm{CD}$ spectra are normalized to their maximum amplitude as shown in fig. S.3b. For a quantitative comparison, we decomposed the normalized absorption and CD spectra into a sum of Gaussian functions of the type $\mathrm{G}_{n}(\lambda)=A_{n} \exp \left[-\left(\left(\lambda-\lambda_{n}\right) / c_{n}\right)^{2}\right]$. The fits for $\mathrm{Fe}^{\mathrm{CIP}}$ are

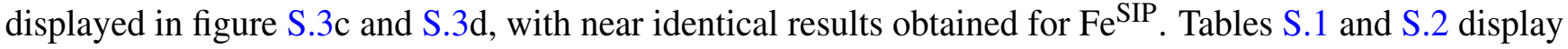
the fit parameters corresponding to the Gaussian functions constituting the bands associated with the LC transitions. For the absorption spectra, a hypsochromic shift of approximately $1 \mathrm{~nm}$ is apparent, in addition to a minor increase of the amplitude of the higher energy band. The hypsochromic shift is similarly observed in the CD bands, yet it is striking that only the high-energy band significantly changes in amplitude, displaying a pronounced reduction. 


\begin{tabular}{|l|l|l|l|l|l|l|l|}
\hline Sample & $\mathrm{R}^{2}$ & $A_{1}$ & $\lambda_{1}(\mathrm{~nm})$ & $c_{1}(\mathrm{~nm})$ & $A_{2}$ & $\lambda_{2}(\mathrm{~nm})$ & $c_{2}(\mathrm{~nm})$ \\
\hline $\mathrm{Fe}^{\mathrm{CIP}}$ & 0.9999 & 0.81 & 294.4 & 13.7 & 0.26 & 302.1 & 5.6 \\
\hline $\mathrm{Fe}^{\mathrm{SIP}}$ & 0.9999 & 0.81 & 293.3 & 13.6 & 0.29 & 301.3 & 5.7 \\
\hline
\end{tabular}

Table S.1. Fit parameters obtained from fitting a sum of four Gaussian functions to the normalized absorption spectra of $\mathrm{Fe}^{\mathrm{CIP}}$ and $\mathrm{Fe}^{\mathrm{SIP}}$. Only the parameters associated with the LC absorption band are displayed with $A_{n}$ referring to the amplitude, $\lambda_{n}$ to the center wavelength and $c_{n}$ to the width of the $n^{\text {th }}$ Gaussian function, as defined in the text.

\begin{tabular}{|l|l|l|l|l|l|l|l|l|l|l|}
\hline Sample & $\mathrm{R}^{2}$ & $A_{1}$ & $\lambda_{1}(\mathrm{~nm})$ & $c_{1}(\mathrm{~nm})$ & $A_{2}$ & $\lambda_{2}(\mathrm{~nm})$ & $c_{2}(\mathrm{~nm})$ & $A_{3}$ & $\lambda_{3}(\mathrm{~nm})$ & $c_{3}(\mathrm{~nm})$ \\
\hline $\mathrm{Fe}^{\mathrm{CIP}}$ & 0.9997 & -0.61 & 286.6 & 10.0 & 0.62 & 303.7 & 4.8 & 0.41 & 305.0 & 8.3 \\
\hline $\mathrm{Fe}^{\mathrm{SIP}}$ & 0.9997 & -0.49 & 285.7 & 9.2 & 0.62 & 302.6 & 4.8 & 0.40 & 303.8 & 8.6 \\
\hline
\end{tabular}

Table S.2. Fit parameters obtained from fitting a sum of six Gaussian functions to the normalized CD spectra of $\mathrm{Fe}^{\mathrm{CIP}}$ and $\mathrm{Fe}^{\mathrm{SIP}}$. Only the parameters associated with the $\mathrm{LC}$ absorption band are displayed with $A_{n}$ referring to the amplitude, $\lambda_{n}$ to the center wavelength and $c_{n}$ to the width of the $n^{\text {th }}$ Gaussian function, as defined in the text.
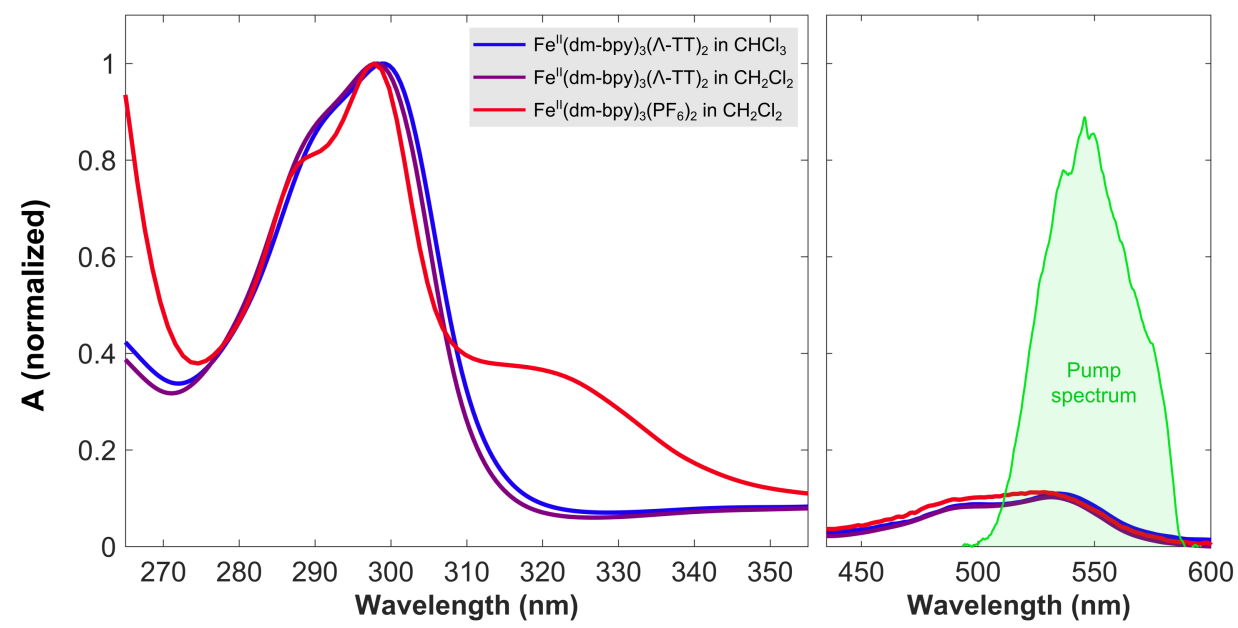

Figure S.1. Steady-state absorption spectra of the three samples investigated in this study. Each spectrum is normalized to its maximum amplitude within the displayed wavelength range. The right panel also includes the spectrum of the excitation pulse employed in the pump-probe experiments. 

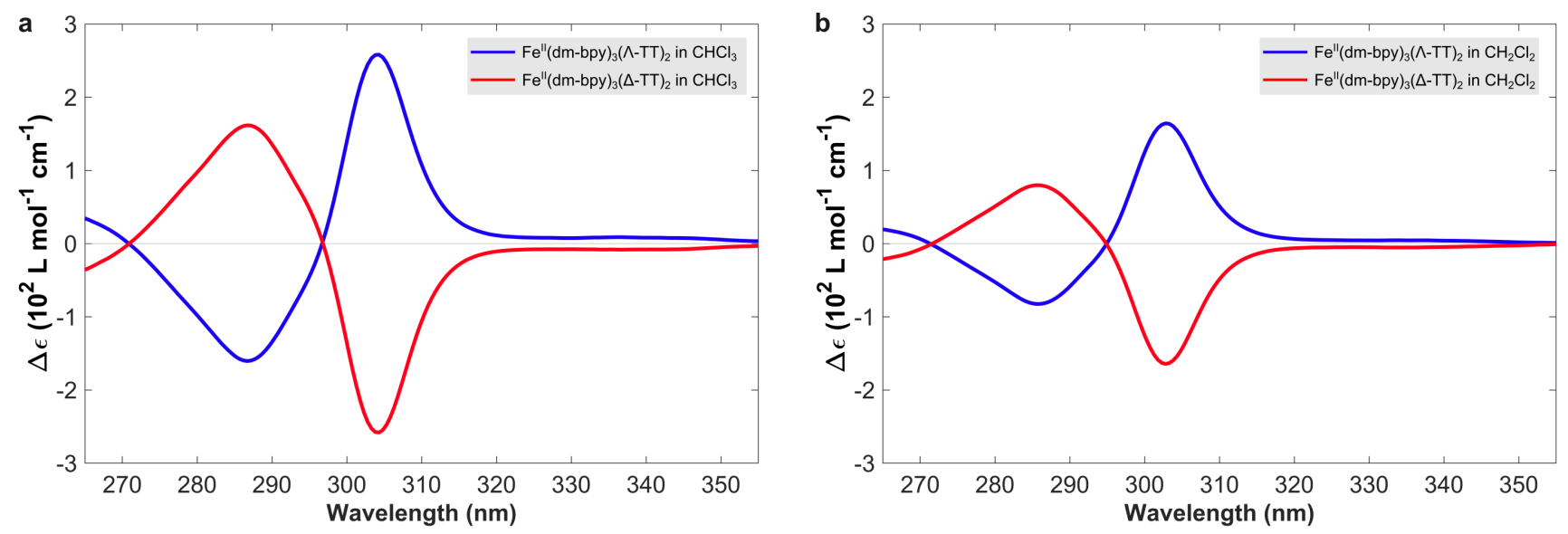

Figure S.2. Steady-state $\mathrm{CD}$ spectra of the $\Lambda$ and $\Delta$ configurations of the diastereomeric ion pairs dissolved in $\mathrm{CHCl}_{3}$ (a) and $\mathrm{CH}_{2} \mathrm{Cl}_{2}$ (b).
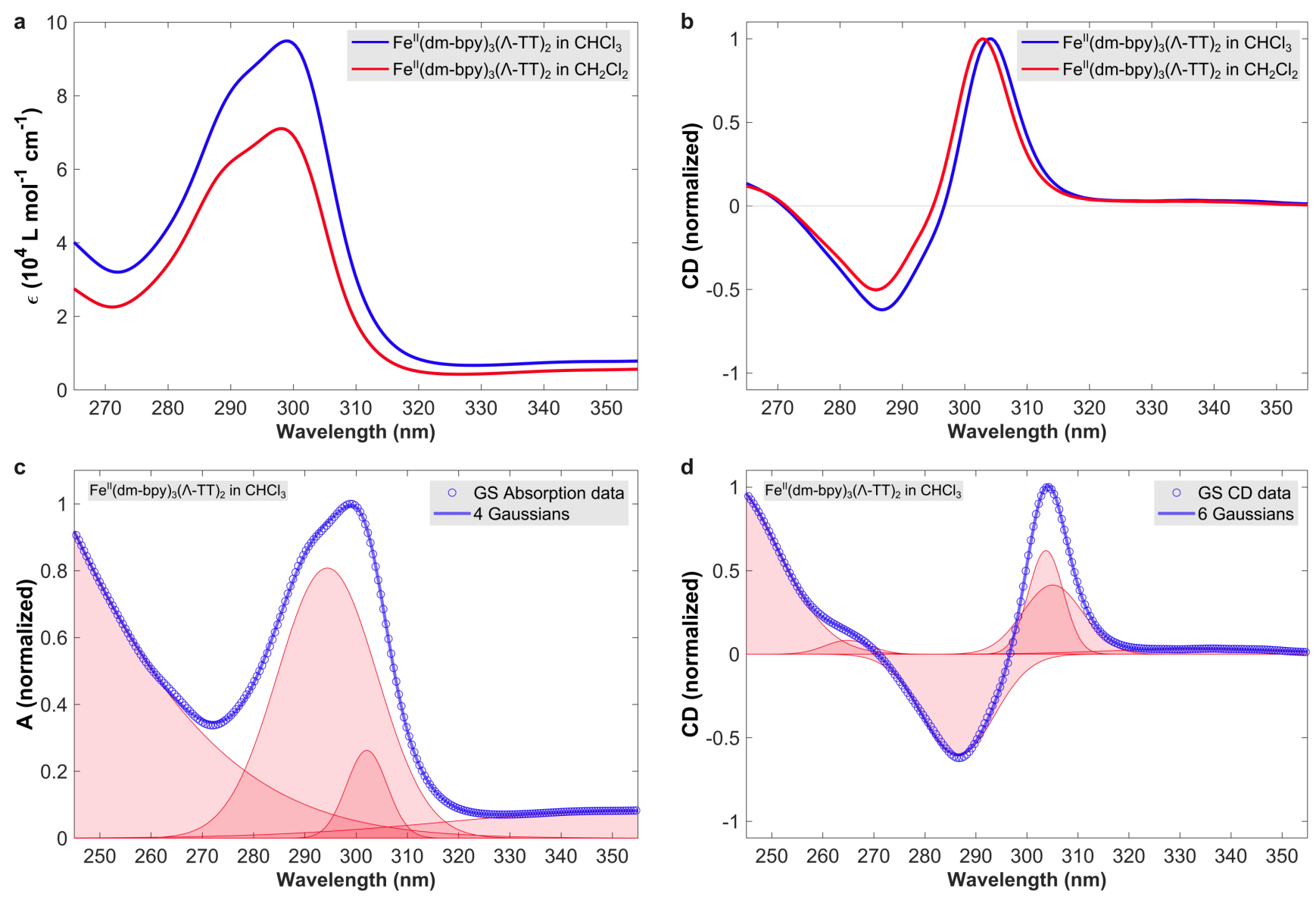

Figure S.3. Detailed comparison of the spectroscopic properties of the diastereomeric ion pair in its $\Lambda$ configuration when dissolved in $\mathrm{CHCl}_{3}$ and $\mathrm{CH}_{2} \mathrm{Cl}_{2}$ : Comparison of molar extinction as a function of wavelength (a), Comparison of CD spectra normalized to their maximum amplitude (b), spectral decomposition of the normalized absorption spectrum in $\mathrm{CHCl}_{3}$ (c), and spectral decomposition of the normalized $\mathrm{CD}$ spectrum in $\mathrm{CHCl}_{3}(\mathrm{~d})$. 


\section{S.2 Transient absorption data and analysis}

The experimental setup and measurement conditions for the transient absorption (TA) experiments are reported in the Methods section. Here, technical details and further data sets are presented to support the discussion in the main article.

Instrument response function. In order to characterize the instrument response function (IRF) of the TA measurements, fig. S.4a diplays the instantaneous response of pure $\mathrm{CHCl}_{3}$ during the temporal overlap of the pump and probe pulses. The onset of the response as a function of probe wavelength describes the chirp of the probe pulse due to the group-velocity dispersion (GVD) from any transmissive optics in the probe beam path and is routinely corrected in the post-processing of TA data ${ }^{\mathrm{si}}$. At each probe wavelength, the nonlinear solvent response is referred to as a coherent artifact (CA), which consists of the two-photon absorption and cross-phase modulation caused by the temporal and spatial overlap of the pump and probe pulses. Fig. S.4c,d displays the CA as a function of pump-probe delay at $290 \mathrm{~nm}$ and $335 \mathrm{~nm}$. To quantify the temporal width of the CA, the absolute value is taken of the TA signal and its local maxima are determined. After cutting the data points between the two outermost maxima, the remaining data is fitted with a Gaussian function. The data points prepared for the fit and the resulting fit functions are displayed in fig. S.4c,d, whereas the associated full-width-half-maximum (FWHM) and fit quality $\left(\mathrm{R}^{2}\right)$ are plotted as a function of probe wavelength in fig. S.4b. The figure displays an average FWHM of approximately 0.3 ps over the whole spectral range, starting at $0.5 \mathrm{ps}$ near $270 \mathrm{~nm}$ and decreasing to 0.2 ps for probe wavelengths $>300 \mathrm{~nm}$. We attribute the larger FWHM at shorter wavelengths to the higher dispersion of transmissive optics in this spectral regime. Note that the fit quality around the chirp's inflection point near $310 \mathrm{~nm}$ is very poor due to additional oscillations and a phase flip in the CA, such that the estimate of the IRF is not very reliable in this region. In this spectral region, we also observe high-frequency oscillations with a low amplitude that persist beyond the probed time window. Fitting the oscillations with a damped sine function results in an oscillation period of approximately $91 \mathrm{fs}$, corresponding to a frequency of 367 $\mathrm{cm}^{-1}$. We attribute this oscillation to the impulsive excitation of a symmetric vibrational mode of the solvent $\left(v_{3}=365 \mathrm{~cm}^{-1}\right)$, which is known to have a lifetime on the picosecond scale ${ }^{\mathrm{si} 8}$. Due to the average IRF of 0.3 ps associated with the CA, we excluded any pump-probe delays shorter than 0.3 ps for the analysis of our TA experiments. 

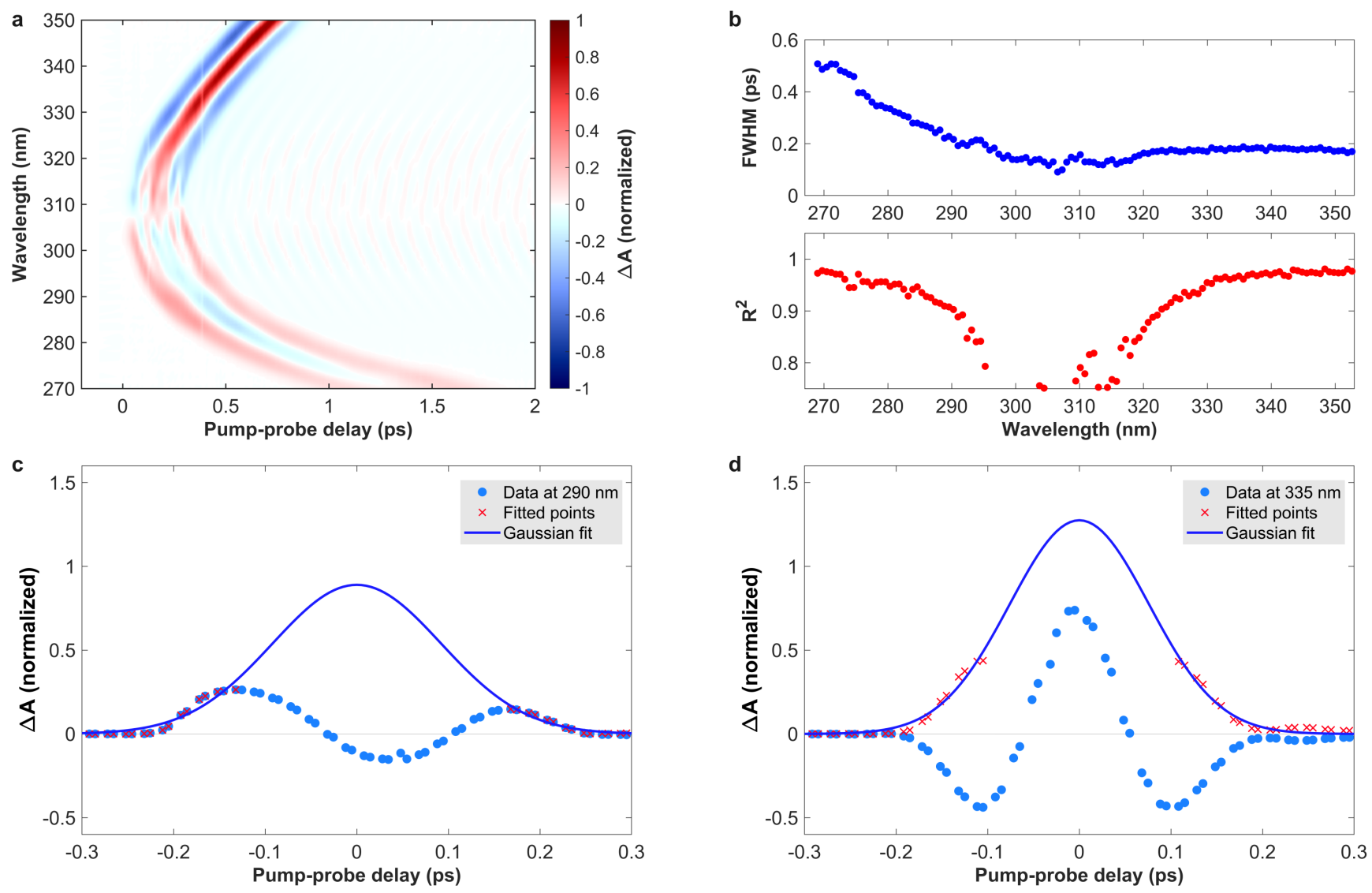

Figure S.4. Transient absorption of pure $\mathrm{CHCl}_{3}$ excited at $530 \mathrm{~mm}$. a) Time-wavelength map of the obtained data without any chirp correction. b) Results from Gaussian fits of the kinetic traces at individual probe wavelengths, displaying the resulting full-width-at-half-maximum (FWHM) and $\mathrm{R}^{2}$ value. c,d) Selected kinetic traces, selected data points to be fitted and the resulting Gaussian fits.

Pump fluence dependence. In order to ensure that the excitation of the MLCT transition takes place in a linear regime with respect to the pump fluence, we recorded TA spectra in $\mathrm{Fe}^{\mathrm{CIP}}$ as a function of pump pulse energy. The pump-probe delay was fixed at $10 \mathrm{ps}$ and the pump pulse energy $E$ was converted to peak fluence $F$ via $F=8 E /\left(w^{2} \pi\right)$, assuming a Gaussian beam with a diameter $w$ at $1 / \mathrm{e}^{2}$ intensity. Here we measured $w=270 \mu \mathrm{m}$. Fig. S.5a displays the acquired spectra, which show isosbestic points at the zero crossings between the GSB and ESA bands and no observable spectral distortions for increasing pump fluence. Fig. S.5b plots the maximum TA amplitude of the main GSB and ESA band averaged over a $2 \mathrm{~nm}$ wide interval as a function of the pump fluence. We performed a linear fit on the data setting the $\mathrm{y}$-intercept to zero and obtained an excellent correspondence with the data as evidenced by the high $\mathrm{R}^{2}$ values displayed in the figure. This confirms that the photoexcitation of the MLCT band takes place in a linear regime for the range of pump fluences measured here. 

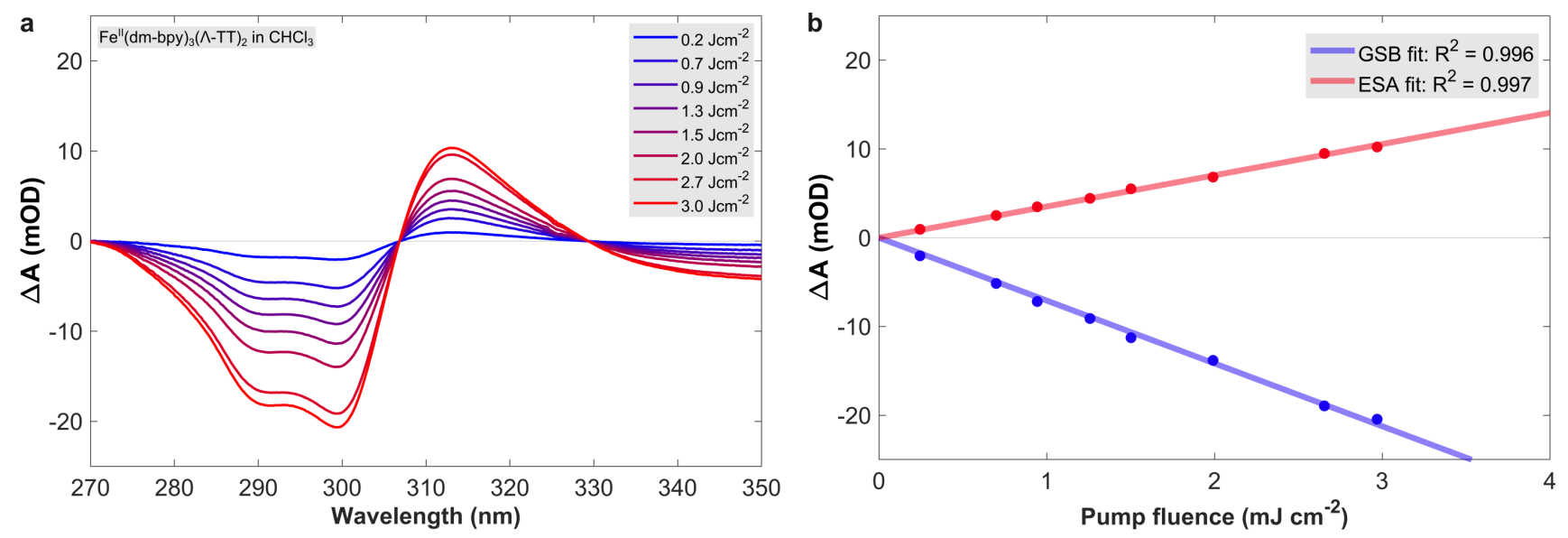

Figure S.5. a) Transient absorption of $\mathrm{Fe}^{\mathrm{II}}(\mathrm{dm}-\mathrm{bpy})_{3}(\Lambda-\mathrm{TT})_{2}$ in $\mathrm{CHCl}_{3}$ as a function of pump fluence at a pump-probe delay of $10 \mathrm{ps}$. b) Maximum transient absorption amplitude of the GSB and ESA band as a function of pump fluence including a linear fit of the data.

TA data. TA measurements of $\mathrm{Fe}^{\mathrm{CIP}}, \mathrm{Fe}^{\mathrm{SIP}}$, and $\mathrm{Fe}^{\mathrm{RIP}}$ were performed back to back under identical

conditions. The pump pulse was centred at approximately $530 \mathrm{~nm}$ (the spectrum is displayed in fig. S.1) and focussed to an average focal spot diameter of $270 \mu \mathrm{m}$ with a peak fluence of $2.8 \mathrm{~mJ} \mathrm{~cm}^{-2}$. The TA spectrum at the so-called magic angle configuration was calculated from consecutively acquired TA spectra with parallel and perpendicular pump and probe polarization configurations. Fig. S.6 displays the GVD-corrected data for all three investigated samples. All samples display a pronounced ground state bleach (GSB) band centred at $295 \mathrm{~nm}$, taking the inverted shape of the steady-state absorption spectrum. Centred near $315 \mathrm{~nm}$, an excited state absorption (ESA) band is observed with a smaller amplitude and an isosbestic point with the GSB band near $305 \mathrm{~nm}$. This ESA band has previously been identified as the red-shifted LC absorption of the photoexcited complex in its HS state ${ }^{\mathrm{s} 9,}$, si10 and thus probes both its population and the ligand system of the excited complex. Similar to the reported case of $\left[\mathrm{Fe}^{\mathrm{II}}(\mathrm{bpy})_{3}\right]^{2+}$ in aqueous solution, the absorption band of the HS state decays simultaneously with the GSB features for all three samples. At wavelengths $>330 \mathrm{~nm}$ a weak and flat GSB band emerges, which extends out of the probed spectral region. The complete time-wavelength maps in fig. S.6b,d,f show pronounced coherent oscillations on the main ESA band and an additional short-lived ESA band $<280 \mathrm{~nm}$.

TA fits. For the quantitative analysis of the TA data the OPTIMUS software package was used to perform global multi-exponential fits ${ }^{\text {si11 }}$. Accounting for the reported photocycle in $\left[\mathrm{Fe}^{\mathrm{II}}(\mathrm{bpy})_{3}\right]^{2+}$, a sequential decay model was employed. To this end, pump-probe delays $<0.3$ ps were excluded from 

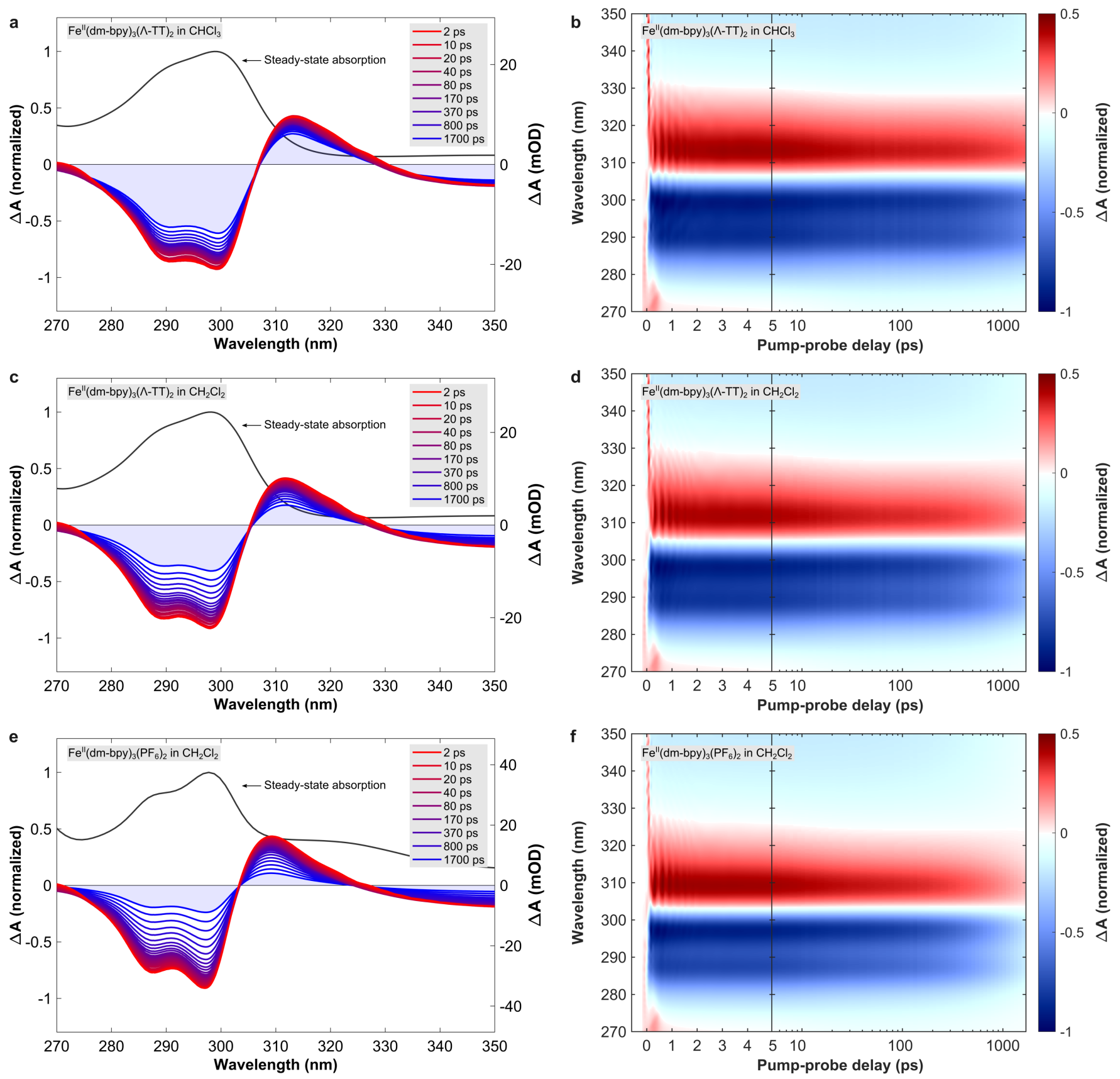

Figure S.6. Transient absorption of $\mathrm{Fe}^{\mathrm{II}}(\mathrm{dm}-\mathrm{bpy})_{3}(\Lambda-\mathrm{TT})_{2}$ in $\mathrm{CHCl}_{3}$ (a,b), in $\mathrm{CH}_{2} \mathrm{Cl}_{2}(\mathrm{c}, \mathrm{d})$, and $\mathrm{Fe}^{\mathrm{II}}(\mathrm{dm}-\mathrm{bpy})_{3}\left(\mathrm{PF}_{6}\right)_{2}$ in $\mathrm{CH}_{2} \mathrm{Cl}_{2}(\mathrm{e}, \mathrm{f})$ after photoexcitation of the MLCT band.

the fit and the GVD-corrected TA data was fit with a series of exponential functions, convoluted with a Gaussian function corresponding to the average IRF of $0.3 \mathrm{ps}$. We found that a minimum number of associated spectra (DAS) and species-associated spectra (SAS), each associated with a time constant. Whereas the DAS of a decay component displays its pre-exponential amplitude as a function of probe 

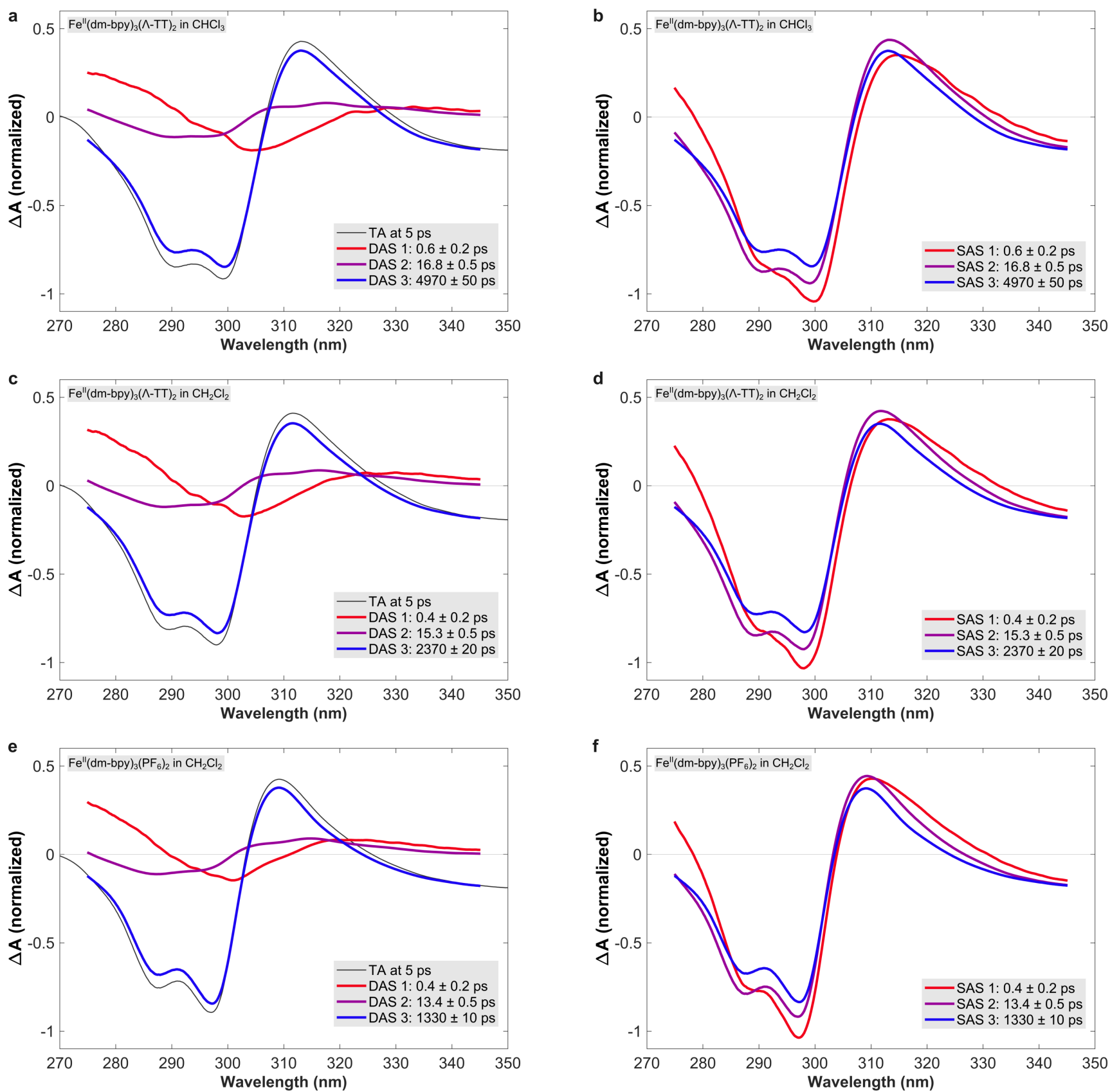

Figure S.7. Decay-associated spectra (left panels) and species-associated spectra (right panels) obtained from a global analysis of the TA data of $\mathrm{Fe}^{\mathrm{II}}(\mathrm{dm}-\mathrm{bpy})_{3}(\Lambda-\mathrm{TT})_{2}$ in $\mathrm{CHCl}_{3}(\mathrm{a}, \mathrm{b})$, in $\mathrm{CH}_{2} \mathrm{Cl}_{2}$ (c,d), and $\mathrm{Fe}^{\mathrm{II}}(\mathrm{dm} \text {-bpy })_{3}\left(\mathrm{PF}_{6}\right)_{2}$ in $\mathrm{CH}_{2} \mathrm{Cl}_{2}$ (e,f) after photoexcitation of the MLCT band. For the plots of the DAS, a TA spectrum at 5 ps is included to show the positions of the GSB and ESA bands. wavelength, its SAS represents the steady-state TA spectrum of the corresponding intermediate state in the sequential model. For the shortest time-constant, an error of \pm 0.2 ps was estimated, accounting for the IRF and the exclusion of pump-probe delays $<0.3 \mathrm{ps.} \mathrm{For} \mathrm{the} \mathrm{remaining} \mathrm{two} \mathrm{time} \mathrm{constants,} \mathrm{the} \mathrm{error}$ was estimated based on the observed variation in the fit parameters resulting from different starting values 


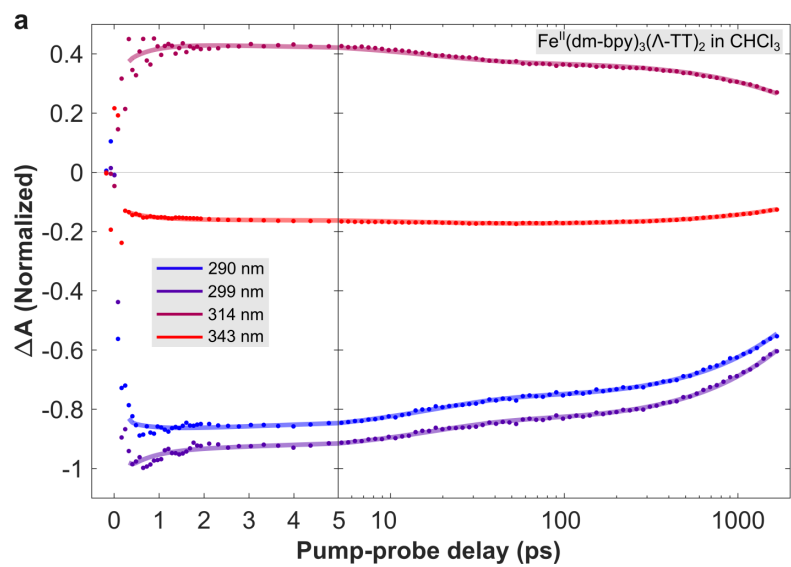

b


e
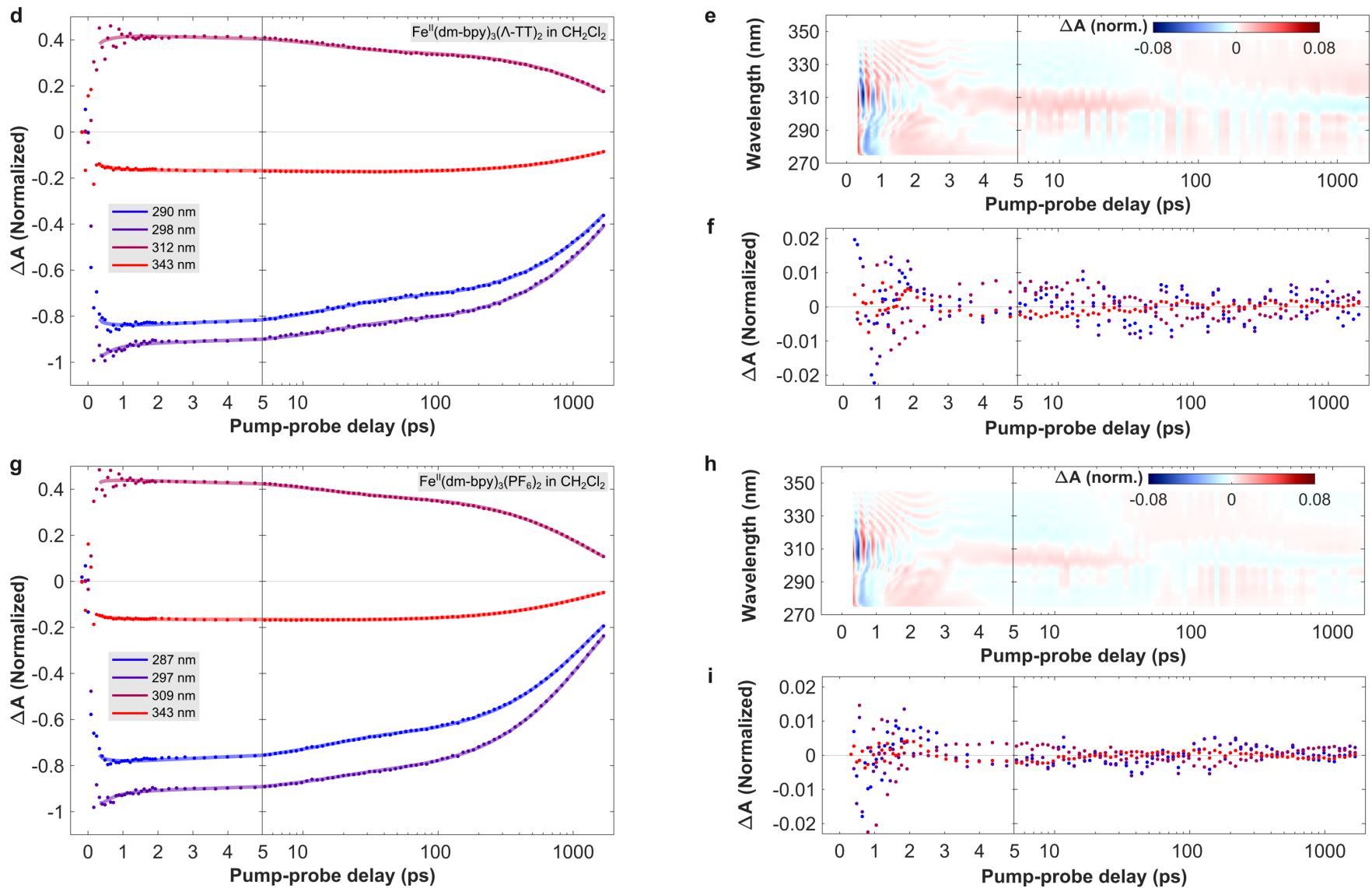

Figure S.8. Evaluation of the quality of the obtained global fits of the TA data for $\mathrm{Fe}^{\mathrm{II}}(\mathrm{dm}-\mathrm{bpy})_{3}(\Lambda-\mathrm{TT})_{2}$ in $\mathrm{CHCl}_{3}(\mathrm{a}, \mathrm{b}, \mathrm{c})$, in $\mathrm{CH}_{2} \mathrm{Cl}_{2}(\mathrm{~d}, \mathrm{e}, \mathrm{f})$, and $\mathrm{Fe}^{\mathrm{II}}(\mathrm{dm}-\mathrm{bpy})_{3}\left(\mathrm{PF}_{6}\right)_{2}$ in $\mathrm{CH}_{2} \mathrm{Cl}_{2}(\mathrm{~h}, \mathrm{i}, \mathrm{j})$. The left panels display the TA amplitude as a function of pump-probe delay at selected probe wavelengths (dots) and the obtained fits (solid lines), whereas the right panels display the time-wavelength map of the residuals (top right) and the residuals for the selected kinetic traces (bottom right).

for the algorithm. Fig. S.8 evaluates the quality of the obtained fits through direct comparison with the data at selected probe wavelengths and through the fit residuals. For the kinetic traces displayed in fig. S.8a,d,g, probe wavelengths near the maximum of the probed GSB and ESA bands were selected. The 
corresponding TA amplitude was averaged over three adjacent detector pixels, corresponding to a spectral width of approximately $0.5 \mathrm{~nm}$. The chosen spectral positions were adapted for each sample and are indicated in the figure legend. In all cases, an excellent correspondence between the data and the fits is observed. Fig. S.8b,e,h displays the obtained residuals over the entire probe wavelength spectrum, whereas fig. S.8c,f,i shows the residuals for the selected kinetic traces. Their low value across the probed spectral and temporal range confirms the high quality of the obtained global fits. Note that the fits do not account for the coherent oscillations in the ESA band for pump-probe delays $<2 \mathrm{ps,} \mathrm{which} \mathrm{are} \mathrm{therefore}$ present in the residuals. For Fe ${ }^{\mathrm{CIP}}$, the obtained time constants are $\tau_{1}^{\mathrm{CIP}}=0.6 \pm 0.2 \mathrm{ps}, \tau_{2}^{\mathrm{CIP}}=16.8 \pm 0.5$ ps, and $\tau_{3}^{\text {CIP }}=4970 \pm 50$ ps. Fig. S.7a,b displays the associated DAS and SAS, with DAS ${ }_{1}$ showing a negative band near $305 \mathrm{~nm}$ and positive bands on either side. The associated spectral changes in the TA data correspond to the transition from $\mathrm{SAS}_{1}$ to $\mathrm{SAS}_{2}$ : a decay of a positive feature $>290 \mathrm{~nm}$ coupled to the rise of a positive feature between $290 \mathrm{~nm}$ and $320 \mathrm{~nm}$, which leads to slight blue-shift of the zero-crossing between the GSB and ESA bands. DAS 2 displays a negative band in the GSB region and a positive band in the ESA region, thereby describing a minor decay of both bands with minor changes of their spectral shapes. $\mathrm{DAS}_{3}$ then takes a shape close to the TA spectrum, implying a simultaneous decay of all TA features to zero with the time constant $\tau_{3}$. Due to the much larger amplitude of $\mathrm{DAS}_{3}$ compared to the other two components, $\tau_{3}$ accounts for most of the decay in the probed TA bands. Compared to Fe $\mathrm{FIP}^{\mathrm{CIP}}$ the global analysis of the TA data for $\mathrm{Fe}^{\mathrm{SIP}}$ and $\mathrm{Fe}^{\mathrm{RIP}}$ gives DAS and SAS with identical spectral features yet successively shorter time constants. For their values we obtain $\tau_{1}^{\mathrm{SIP}}=0.4 \pm 0.2, \tau_{2}^{\mathrm{SIP}}=15.3 \pm 0.5 \mathrm{ps}$ and $\tau_{3}^{\mathrm{SIP}}=2370 \pm 20 \mathrm{ps}$ for Fe${ }^{\mathrm{SIP}}$, and $\tau_{1}^{\mathrm{RIP}}=0.4 \pm 0.2, \tau_{2}^{\mathrm{RIP}}=13.4 \pm 0.5$ ps and $\tau_{3}^{\mathrm{RIP}}=1330 \pm 10 \mathrm{ps}$ for $\mathrm{Fe}^{\mathrm{RIP}}$. This indicates that the same relaxation processes are taking place on faster time scales.

TA of $\mathrm{Fe}^{\mathrm{II}}(\mathrm{bpy})_{3} \mathrm{Cl}_{2}$ in $\mathrm{H}_{2} \mathrm{O}$. In order to unambiguously assign the excited state dynamics in the investigated samples, we conducted benchmark measurements of the well-studied $\mathrm{Fe}^{\mathrm{II}}(\mathrm{bpy})_{3} \mathrm{Cl}_{2}$ in $\mathrm{H}_{2} \mathrm{O}$. The experimental conditions and data analysis procedures were identical to those for Fe ${ }^{\mathrm{CIP}}, \mathrm{Fe}^{\mathrm{SIP}}$, and $\mathrm{Fe}^{\mathrm{RIP}}$. Fig. S.9a displays the TA time-wavelength map, normalized to the maximum GSB signal, whereas fig. S.9b shows the DAS obtained from a sequential multi-exponential fit convoluted with the IRF of the measurement. Fig. S.9c displays the TA amplitude as a function of pump-probe delay at selected probe wavelengths and includes the obtained fit functions as solid lines, whereas fig. S.9d,e 


\begin{tabular}{|c|c|c|c|c|c|c|}
\hline Sample & $\tau_{1}(\mathrm{ps})$ & $\tau_{2}(\mathrm{ps})$ & $\tau_{3}(\mathrm{ps})$ & $\rho_{1}(\mathrm{ps})$ & $\rho_{2}(\mathrm{ps})$ & $\phi(\mathrm{ps})$ \\
\hline$\Lambda-\mathrm{Fe}^{\mathrm{CIP}}$ & $0.6 \pm 0.2$ & $16.8 \pm 0.5$ & $4970 \pm 50$ & $4.8 \pm 0.2$ & $520 \pm 10$ & $80 \pm 10$ \\
\hline$\Lambda-\mathrm{Fe}^{\mathrm{SIP}}$ & $0.4 \pm 0.2$ & $15.3 \pm 0.5$ & $2370 \pm 20$ & $3.6 \pm 0.2$ & $260 \pm 10$ & $70 \pm 10$ \\
\hline $\mathrm{Fe}^{\mathrm{RIP}}$ & $0.4 \pm 0.2$ & $13.4 \pm 0.5$ & $1330 \pm 10$ & $1.6 \pm 0.2$ & $110 \pm 10$ & - \\
\hline $\mathrm{Fe}^{\mathrm{II}}(\mathrm{bpy})_{3} \mathrm{Cl}_{2}$ in $\mathrm{H}_{2} \mathrm{O}$ & $0.4 \pm 0.2$ & $3.3 \pm 0.2$ & $680 \pm 10$ & - & - & - \\
\hline
\end{tabular}

Table S.3. Time constants obtained from various multi-exponential fits of the time-resolved data from all conducted experiments. The constants $\tau_{i}(\mathrm{i}=1,2,3)$ were obtained from the TA experiments, whereas $\rho_{i}$ and $\phi$ were obtained from the TAA and TRCD experiments, respectively. 

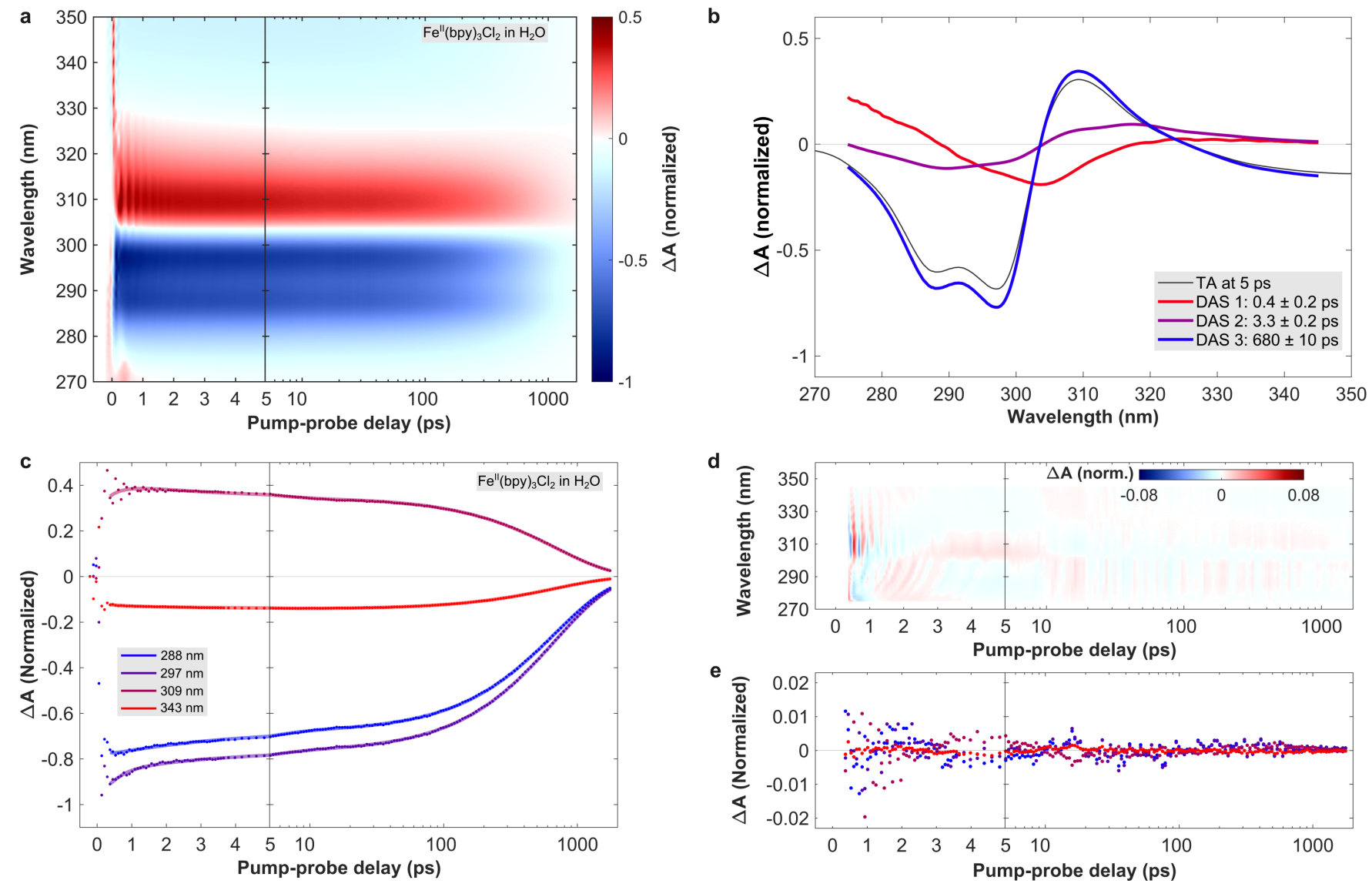

Figure S.9. Transient absorption of $\mathrm{Fe}^{\mathrm{II}}(\mathrm{bpy})_{3} \mathrm{Cl}_{2}$ in $\mathrm{H}_{2} \mathrm{O}$, after photoexcitation of the MLCT band near $530 \mathrm{~nm}$ (a) and the decay-associated spectra obtained from a global analysis of the TA data (b). Evaluation of the quality of the obtained global fits by comparing the TA data at selected probe wavelengths (dots) to the fits (solid lines) (c) and by displaying the obtained fit residuals (d,e). 

samples.
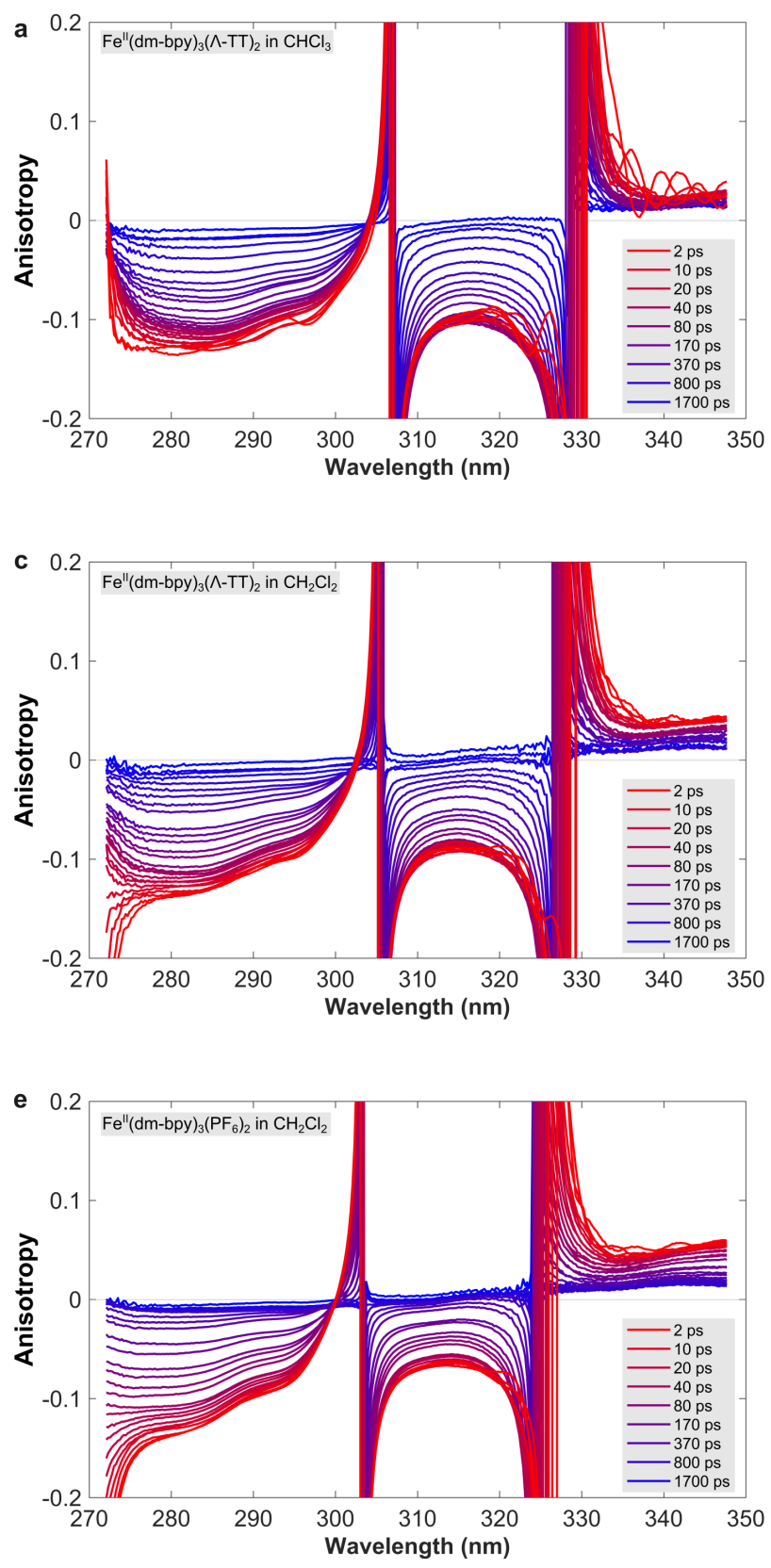
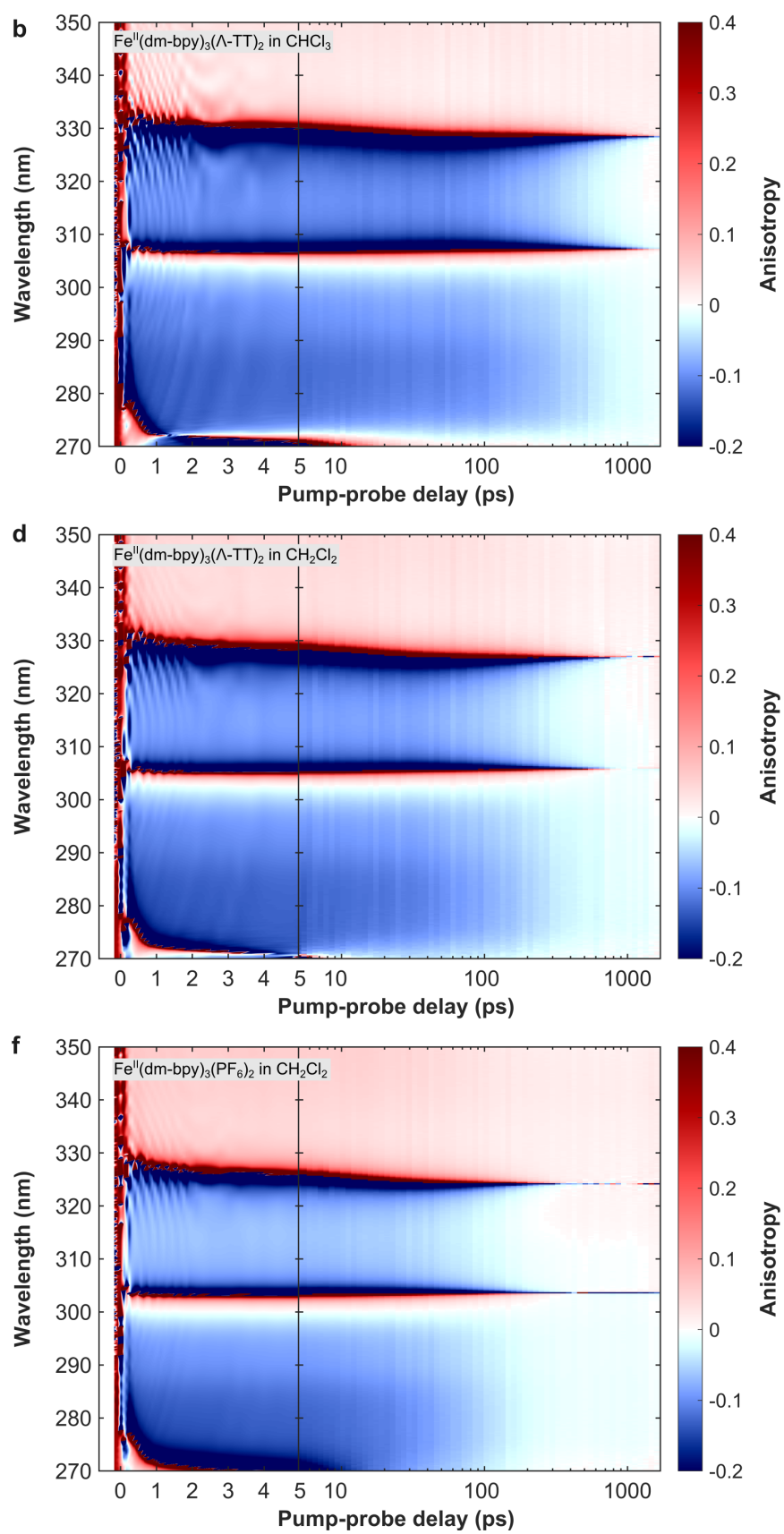

Figure S.10. Transient absorption anisotropy of $\mathrm{Fe}^{\mathrm{II}}(\mathrm{dm}-\mathrm{bpy})_{3}(\Lambda-\mathrm{TT})_{2}$ in $\mathrm{CHCl}_{3}(\mathrm{a}, \mathrm{b})$, in $\mathrm{CH}_{2} \mathrm{Cl}_{2}(\mathrm{c}, \mathrm{d})$, and $\mathrm{Fe}^{\mathrm{II}}(\mathrm{dm}-\mathrm{bpy})_{3}\left(\mathrm{PF}_{6}\right)_{2}$ in $\mathrm{CH}_{2} \mathrm{Cl}_{2}(\mathrm{e}, \mathrm{f})$ after photoexcitation of the MLCT band. 
TAA fits. For the quantitative analysis of the TAA data the OPTIMUS software package was used to perform global multi-exponential fits. As the calculation of the TAA spectra involves the TA spectrum as a denominator, the TAA amplitude diverges when the TA amplitude takes values close to zero. This is the case at probe wavelengths near zero-crossings between GSB and ESA bands, at early pump-probe delays due to cross-phase modulation (see section on the IRF determination) and at late pump-probe delays where the excited state population approaches zero. For the performed global fits we accounted for all three factors by excluding probe wavelengths near zero-crossings and by excluding pump-probe delays $<0.4 \mathrm{ps}$. Since each sample displayed different excited state lifetimes, the longest pump-probe delay $t_{\max }$ included in the global fit was adapted accordingly, such that $\mathrm{t}_{\max }^{\mathrm{CIP}} \approx 1000 \mathrm{ps}, \mathrm{t}_{\max }^{\mathrm{SIP}} \approx 500 \mathrm{ps}$, and $\mathrm{t}_{\max }^{\mathrm{RIP}} \approx 200 \mathrm{ps}$. We found that a minimum number of two exponential functions are required to fit the TAA data, both in a fit model with parallel and sequential decays. Here we found identical time constants and DAS independent of the fit model, which is typically the case when the obtained time constants differ by more than an order of magnitude. Fig. S.11 thus only displays the obtained DAS and SAS for a sequential fit model. Note that their spectral widths directly denote the probe wavelength ranges that were included in the global fits of each sample. The error ranges for the obtained time constants were estimated by observing their variation when shifting the included probe wavelength region and pump-probe delay $t_{\max }$. Fig. S.12 evaluates the quality of the obtained fits through direct comparison with the data at selected probe wavelengths and the fit residuals. For the kinetic traces displayed in fig. S.12a,d,g, probe wavelengths near the maximum of the probed GSB and ESA bands were selected. The corresponding TAA amplitude was averaged over three adjacent detector pixels, corresponding to a spectral width of approximately $0.5 \mathrm{~nm}$. The chosen spectral positions were adapted for each sample and are indicated in the figure legend. In all cases, a very good correspondence between the data and the fits is observed. Fig. S.8b,e,h displays the obtained residuals over the included probe wavelength ranges, whereas fig. S.8c,f,i shows the residuals for the selected kinetic traces. Their comparatively low values across the included spectral and temporal range confirms the high quality of the obtained global fits. Note that the residuals in the ESA band display oscillations for pump-probe delays $<2 \mathrm{ps}$, which are also visible in the raw data in fig. S.10. We attribute these oscillations to the coherent oscillations observed in the ESA band's TA kinetics, which are not accounted for by the global fits of the TAA data. 

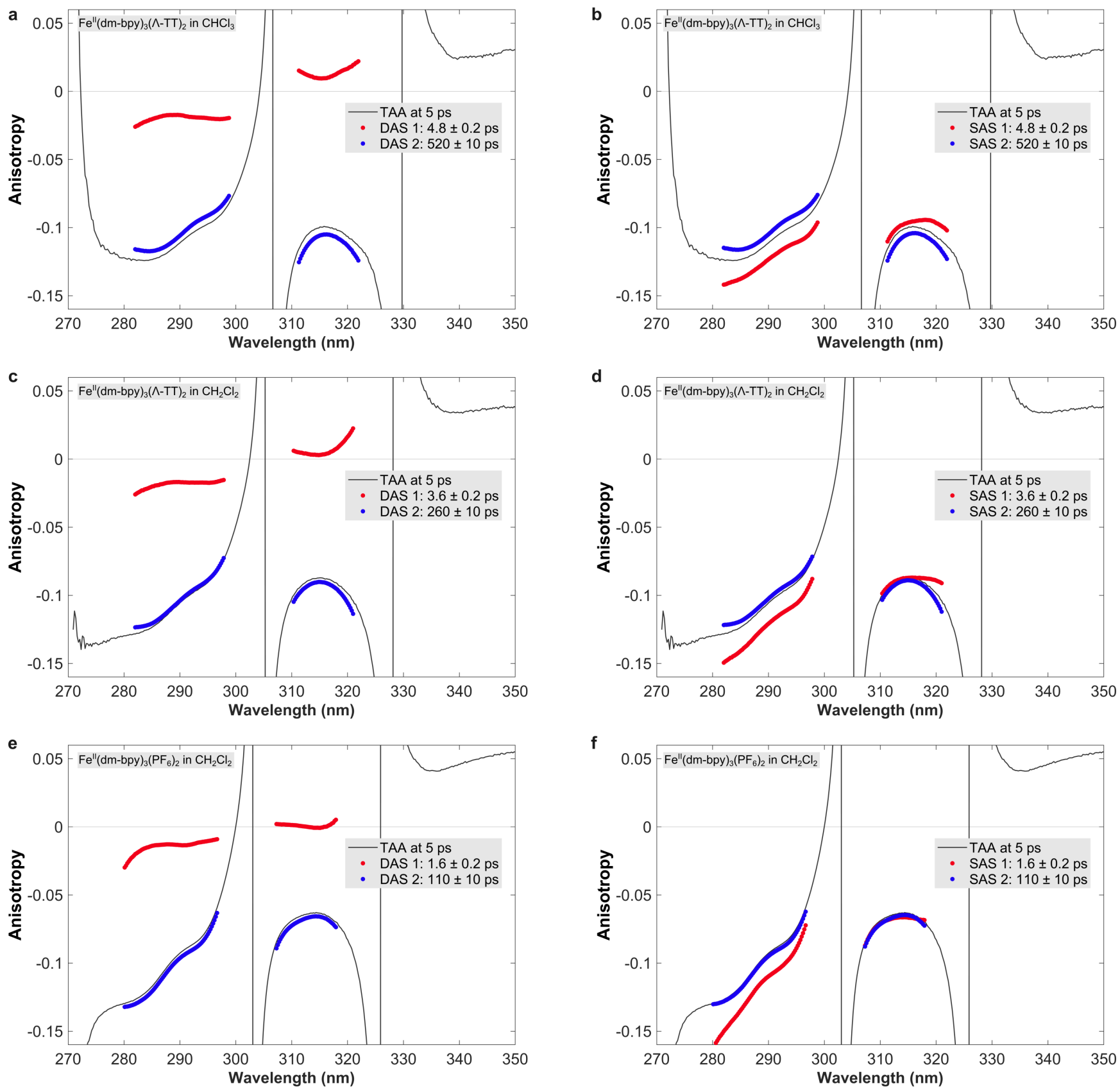

Figure S.11. Decay-associated spectra (left panels) and species-associated spectra (right panels) obtained from a global analysis of the TAA data of $\mathrm{Fe}^{\mathrm{II}}(\mathrm{dm}-\mathrm{bpy})_{3}(\Lambda-\mathrm{TT})_{2}$ in $\mathrm{CHCl}_{3}(\mathrm{a}, \mathrm{b})$, in $\mathrm{CH}_{2} \mathrm{Cl}_{2}$ $(\mathrm{c}, \mathrm{d})$, and $\mathrm{Fe}^{\mathrm{II}}(\mathrm{dm}-\mathrm{bpy})_{3}\left(\mathrm{PF}_{6}\right)_{2}$ in $\mathrm{CH}_{2} \mathrm{Cl}_{2}(\mathrm{e}, \mathrm{f})$ after photoexcitation of the MLCT band. For both the DAS and the SAS, a TAA spectrum at $5 \mathrm{ps}$ is included to show the positions of the main TAA bands and zero-crossings.

TAA calculations. For the interpretation of the TAA data, we calculated the TAA values expected

from the octahedral coordination environment of a tris-chelate complex. We then calculated the TAA as a function of the angular deviation of the ligands from this configuration and estimated the average TAA 


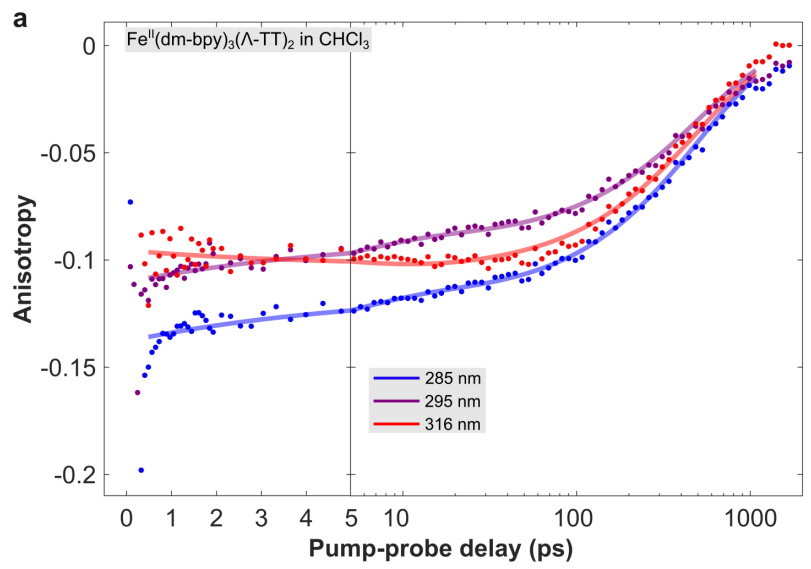

b

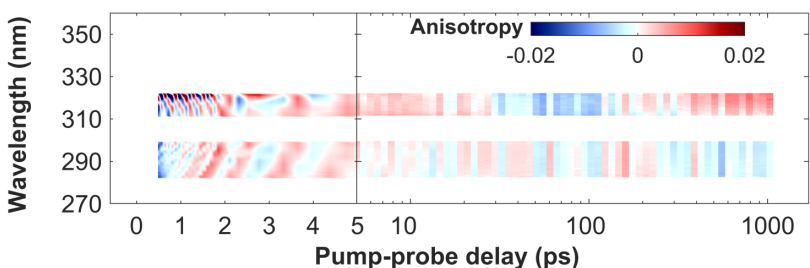

C
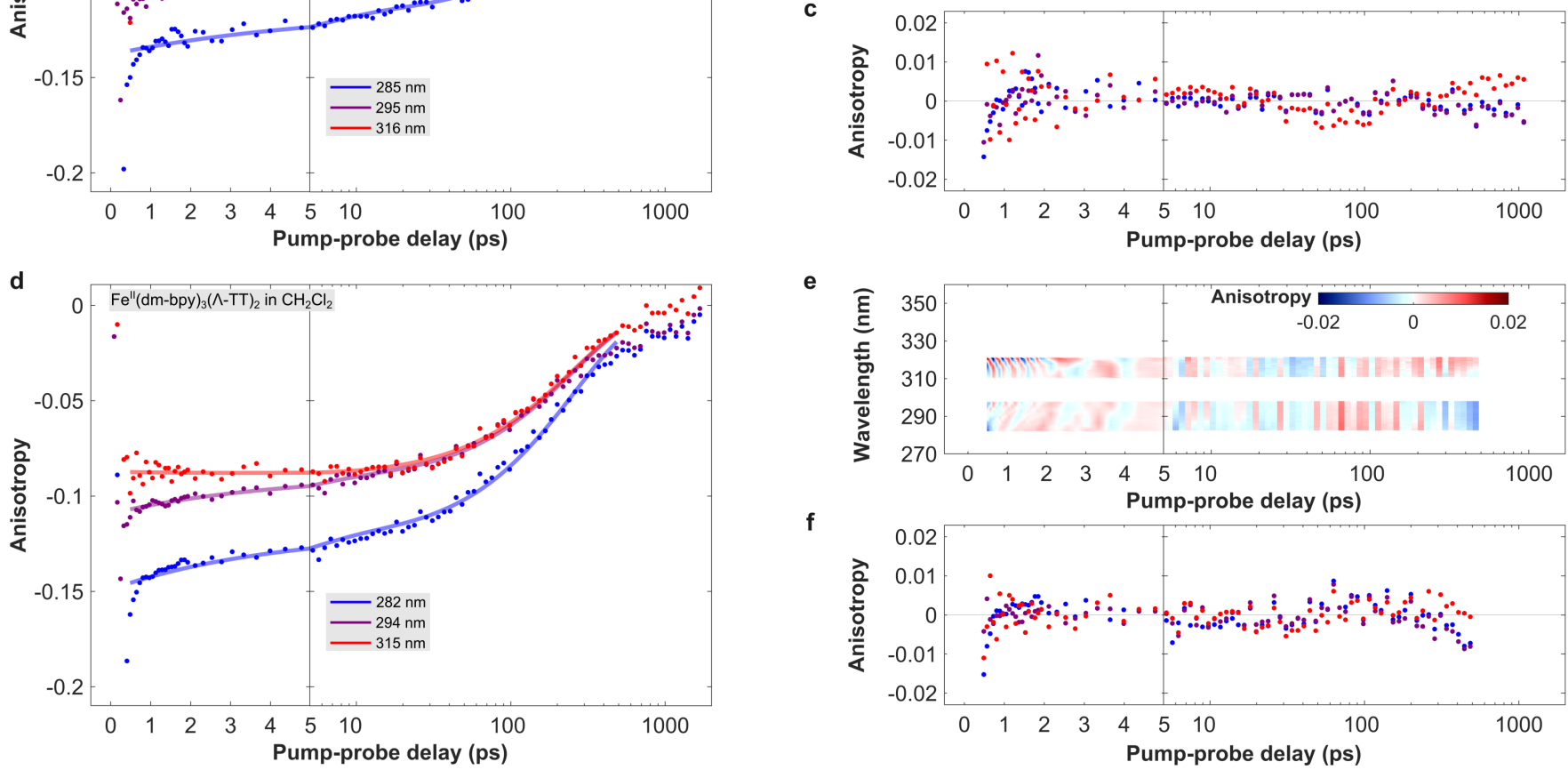

e
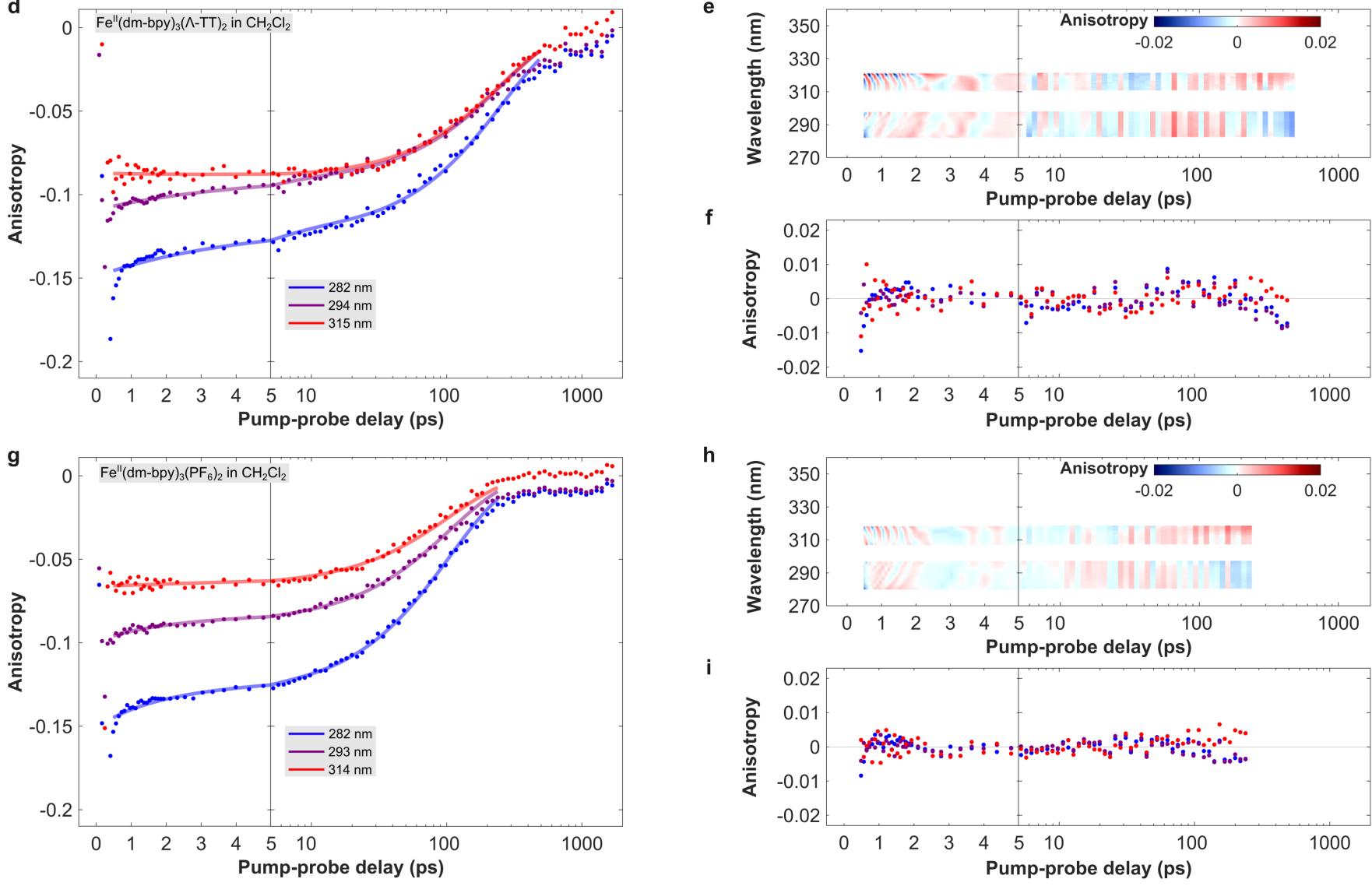

Figure S.12. Evaluation of the quality of the obtained global fits of the TAA data for $\mathrm{Fe}^{\mathrm{II}}(\mathrm{dm}-\mathrm{bpy})_{3}(\Lambda-\mathrm{TT})_{2}$ in $\mathrm{CHCl}_{3}(\mathrm{a}, \mathrm{b}, \mathrm{c})$, in $\mathrm{CH}_{2} \mathrm{Cl}_{2}(\mathrm{~d}, \mathrm{e}, \mathrm{f})$, and $\mathrm{Fe}^{\mathrm{II}}(\mathrm{dm}-\mathrm{bpy})_{3}\left(\mathrm{PF}_{6}\right)_{2}$ in $\mathrm{CH}_{2} \mathrm{Cl}_{2}(\mathrm{~g}, \mathrm{~h}, \mathrm{i})$. The left panels display the TA amplitude as a function of pump-probe delay at selected probe wavelengths (dots) and the obtained fits (solid lines), whereas the right panels display the time-wavelength map of the residuals (top right) and the residuals for the selected kinetic traces (bottom right).

resulting from an increasingly broader conformational distribution. To this end, we take the procedure developed by Wallin and co-workers for an iso-structural $\left[\mathrm{Ru}(\mathrm{bpy})_{3}\right]^{2+}$ complex as a starting point ${ }^{\text {si12 }}$ and then add the required rotational degrees of freedom. The inset in fig. S.13a displays the chosen coordinate 
system and the resulting arrangement of the relevant MLCT and LC transition dipoles denoted as $\overrightarrow{\mathrm{M}}_{i}$ and $\overrightarrow{\mathrm{L}}_{i}$, respectively. Here subscript $i=1,2,3$ is associated with the ligands of the tris-chelate complex as shown in the coordinate system. Note that for each ligand, both the MLCT and LC transition dipoles are confined to the ligand plane and are oriented perpendicularly to each other. Whilst the MLCT transition is directed away from the metal centre, the LC transition is assumed to be aligned along the long-axis of the bipyridine ligand. Within this reference frame, we obtain the following vector expressions for the transition dipoles in their original octahedral arrangement:

$$
\begin{aligned}
& \overrightarrow{\mathrm{M}}_{1}=\frac{1}{\sqrt{2}}\left(\hat{e}_{x}-\hat{e}_{y}\right), \quad \overrightarrow{\mathrm{L}}_{1}=\frac{1}{\sqrt{2}}\left(\hat{e}_{x}+\hat{e}_{y}\right) \\
& \overrightarrow{\mathrm{M}}_{2}=\frac{1}{\sqrt{2}}\left(-\hat{e}_{x}+\hat{e}_{z}\right), \quad \overrightarrow{\mathrm{L}}_{2}=\frac{1}{\sqrt{2}}\left(\hat{e}_{x}+\hat{e}_{z}\right) \\
& \overrightarrow{\mathrm{M}}_{3}=\frac{1}{\sqrt{2}}\left(\hat{e}_{y}-\hat{e}_{z}\right), \quad \overrightarrow{\mathrm{L}}_{3}=\frac{1}{\sqrt{2}}\left(\hat{e}_{y}+\hat{e}_{z}\right)
\end{aligned}
$$

Note that all vectors are of unit length. We now introduce two rotational degrees of freedom to each LC transition dipole: 1) a rotation angle $\theta$ of $\overrightarrow{\mathrm{L}}_{i}$ out of its ligand plane with $\overrightarrow{\mathrm{M}}_{i}$ as the rotation axis, and 2) a rotation angle $\delta$ of $\overrightarrow{\mathrm{L}}_{i}$ in its ligand plane around the metal-centre. We can then use the standard rotation matrices $\mathbf{R}_{x}(\boldsymbol{\delta}), \mathbf{R}_{y}(\boldsymbol{\delta}), \mathbf{R}_{z}(\boldsymbol{\delta})$ to perform the rotation of $\overrightarrow{\mathrm{L}}_{i}$ by $\delta$ around the respective coordinate axes, and apply the well known Rodrigues' rotation formula to perform a rotation of $\overrightarrow{\mathrm{L}}_{i}$ around $\overrightarrow{\mathrm{M}}_{i}$ by an angle $\theta$. We then obtain the following expressions for the rotated LC transition dipoles $\overrightarrow{\mathrm{L}}_{i}(\boldsymbol{\theta}, \boldsymbol{\delta})$ :

$$
\begin{aligned}
& \overrightarrow{\mathrm{L}}_{1}(\theta, \delta)=\mathbf{R}_{z}(\delta) \cdot\left(\overrightarrow{\mathrm{L}}_{1} \cos \theta+\left(\overrightarrow{\mathrm{M}}_{1} \times \overrightarrow{\mathrm{L}}_{1}\right) \sin \theta+\overrightarrow{\mathrm{M}}_{1}\left(\overrightarrow{\mathrm{M}}_{1} \cdot \overrightarrow{\mathrm{L}}_{1}\right)(1-\cos \theta)\right) \\
& \overrightarrow{\mathrm{L}}_{2}(\theta, \delta)=\mathbf{R}_{y}(\boldsymbol{\delta}) \cdot\left(\overrightarrow{\mathrm{L}}_{2} \cos \theta+\left(\overrightarrow{\mathrm{M}}_{2} \times \overrightarrow{\mathrm{L}}_{2}\right) \sin \theta+\overrightarrow{\mathrm{M}}_{2}\left(\overrightarrow{\mathrm{M}}_{2} \cdot \overrightarrow{\mathrm{L}}_{2}\right)(1-\cos \theta)\right) \\
& \overrightarrow{\mathrm{L}}_{3}(\theta, \delta)=\mathbf{R}_{x}(\boldsymbol{\delta}) \cdot\left(\overrightarrow{\mathrm{L}}_{3} \cos \theta+\left(\overrightarrow{\mathrm{M}}_{3} \times \overrightarrow{\mathrm{L}}_{3}\right) \sin \theta+\overrightarrow{\mathrm{M}}_{3}\left(\overrightarrow{\mathrm{M}}_{3} \cdot \overrightarrow{\mathrm{L}}_{3}\right)(1-\cos \theta)\right)
\end{aligned}
$$

Without loss of generality, we now assume that the initial photoexcitation takes place via $\overrightarrow{\mathrm{M}}_{1}$. The expected TAA value obtained from a single LC transition dipole rotated by $\theta$ and $\delta$ from its original 

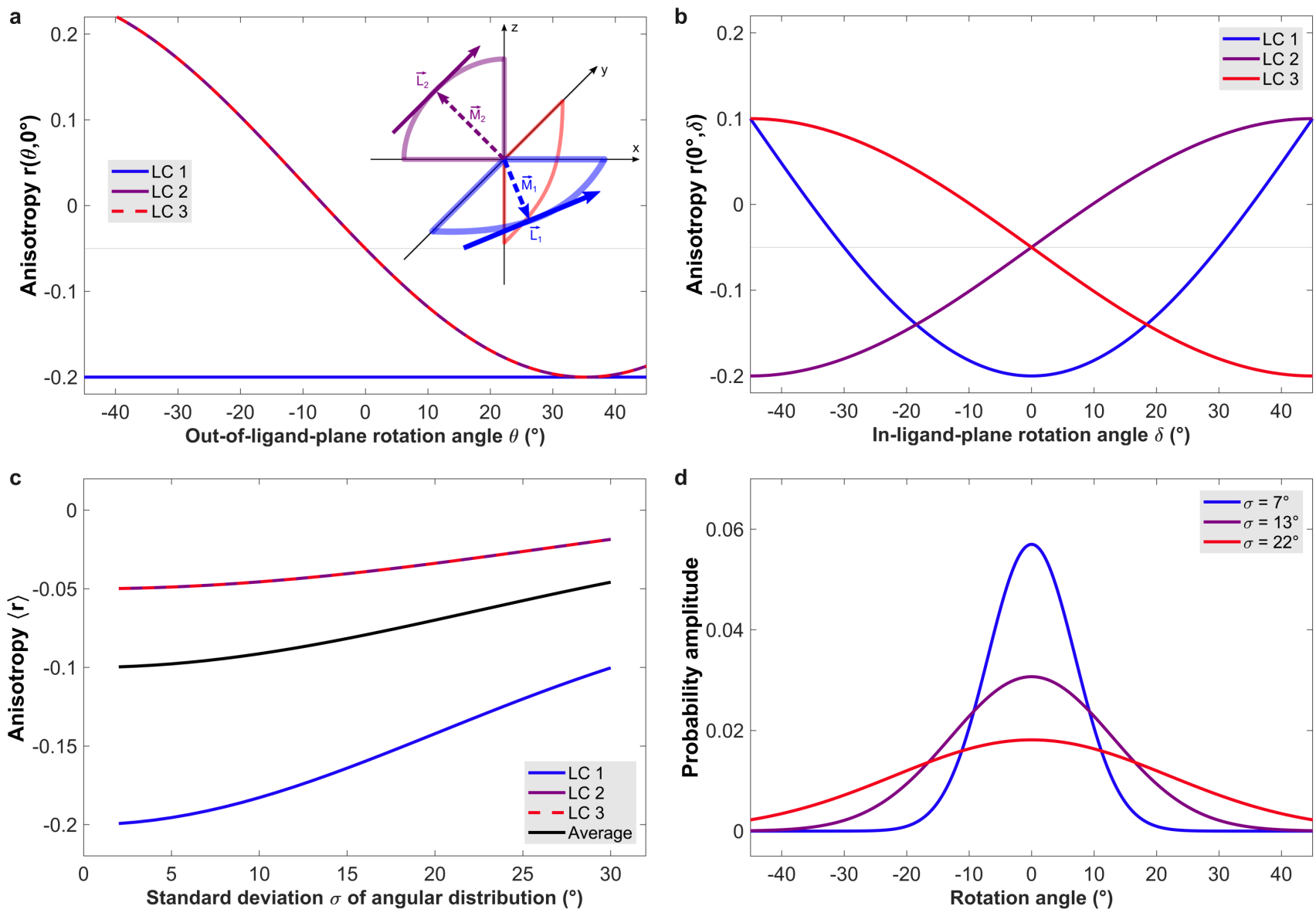

Figure S.13. Calculation of expected anisotropy values from the long-axis ligand centred (LC) transition dipoles of a tris-chelate complex photoexcited via one of its MLCT transitions (here: $\overrightarrow{\mathrm{M}}_{1}$ on ligand 1). a) Anisotropy calculated for individual LC transition dipoles as a function of their out-of-ligand-plane rotation angle. The inset displays the employed coordinate system and the labelling of the transition dipoles. b) Anisotropy calculated for individual LC transition dipoles as a function of their in-ligand-plane rotation angle around the origin of the coordinate system. c) Anisotropy obtained from a conformational ensemble over the in- and out-of-ligand-plane rotation angles as a function of the standard deviation of the associated two-dimensional Gaussian distribution. The average anisotropy value (solid black line) corresponds to the value obtained in an experimental measurement. d) One-dimensional Gaussian distributions with selected standard deviations, illustrating the conformational ensembles associated with the anisotropy values measured in the presented experiments.

octahedral arrangement can now be expressed as:

$$
\mathrm{r}_{i}(\theta, \delta)=\frac{3\left[\overrightarrow{\mathrm{M}}_{1} \cdot \overrightarrow{\mathrm{L}}_{i}(\theta, \delta)\right]^{2}-1}{5}
$$

Fig. S.13a displays the resulting TAA values for all three $\mathrm{LC}$ transition dipoles as function of $\theta$ whilst 
keeping $\delta=0^{\circ}$. For ligand 1 , any out-of-ligand-plane rotation of $\overrightarrow{\mathrm{L}}_{1}$ preserves the perpendicular alignment with $\vec{M}_{1}$, such that $r_{1}(\theta, 0)=-0.2$, as expected. Due to the symmetry of the complex, we then obtain $r_{2}\left(\theta, 0^{\circ}\right)=r_{3}\left(\theta, 0^{\circ}\right)$, with $r_{2}\left(0^{\circ}, 0^{\circ}\right)=r_{3}\left(0^{\circ}, 0^{\circ}\right)=-0.05$ for the original octahedral arrangement. Importantly, we can directly see that $r_{2}\left(\theta, 0^{\circ}\right)$ and $r_{3}\left(\theta, 0^{\circ}\right)$ are not symmetric with respect to $\theta=0^{\circ}$, such that $\frac{1}{2}\left(\mathrm{r}_{2,3}\left(\theta, 0^{\circ}\right)+\mathrm{r}_{2,3}\left(-\theta, 0^{\circ}\right)\right)>-0.05$ for the angular range plotted here $\left(|\theta|<45^{\circ}\right)$. This implies that any symmetric conformational distribution around $\theta=0^{\circ}$ will lead to an increase in the average TAA measured from $\overrightarrow{\mathrm{L}}_{2,3}$, with the TAA value obtained from $\overrightarrow{\mathrm{L}}_{1}$ being unaffected by the distribution.

Fig. S.13b shows the analogous TAA values for all three LC transition dipoles as function of $\delta$ and keeping $\theta=0^{\circ}$. For ligand 1 , any in-ligand-plane rotation of $\overrightarrow{\mathrm{L}}_{1}$ will move its orientation away from a perpendicular towards a parallel orientation with $\overrightarrow{\mathrm{M}}_{1}$. This implies that $\frac{1}{2}\left(\mathrm{r}_{1}\left(0^{\circ}, \delta\right)+\mathrm{r}_{1}\left(0^{\circ},-\delta\right)\right)>-0.2$ for the considered angular range $\left(|\delta|<45^{\circ}\right)$. Both $\mathrm{r}_{2,3}\left(0^{\circ}, \delta\right)$ are now symmetric with respect to $\delta=0^{\circ}$, such that $\frac{1}{2}\left(\mathrm{r}_{2,3}\left(0^{\circ}, \delta\right)+\mathrm{r}_{2,3}\left(0^{\circ},-\delta\right)\right)=-0.05$ for the considered angular range. This implies that any symmetric conformational distribution around $\delta=0^{\circ}$ will lead to an increase in the average TAA measured from $\overrightarrow{\mathrm{L}}_{1}$, with the TAA values obtained from $\overrightarrow{\mathrm{L}}_{2,3}$ remaining unaffected by the distribution. Combined with the results obtained for out-of-plane rotations, we can summarize that any rotationally symmetric conformational ensemble around $\theta=\delta=0^{\circ}$ will increase the measured TAA for each of the three LC transitions.

To gain further quantitative information, we calculated the TAA values for each LC transition, assuming a two-dimensional Gaussian distribution of rotation angles around $\theta=\delta=0^{\circ}$ in each case. For the conformational averages of the resulting TAA values we then obtain:

$$
\left\langle\mathrm{r}_{i}\right\rangle\left(\sigma_{\theta}, \sigma_{\delta}\right)=\frac{1}{2 \pi \sigma_{\theta} \sigma_{\delta}} \int_{-\theta_{0}}^{\theta_{0}} \int_{-\delta_{0}}^{\delta_{0}} \mathrm{r}_{i}(\theta, \delta) \exp \left\{-\left(\frac{\theta^{2}}{2 \sigma_{\theta}^{2}}+\frac{\delta^{2}}{2 \sigma_{\delta}^{2}}\right)^{2}\right\} d \theta d \delta
$$

where $\sigma_{\theta}, \sigma_{\delta}$ denote the standard deviations of the Gaussian distribution and $\left[-\theta_{0}, \theta_{0}\right],\left[-\delta_{0}, \delta_{0}\right]$ the numerical integration boundaries for the calculation. We then calculated $\left\langle\mathrm{r}_{i}\right\rangle\left(\sigma_{\theta}, \sigma_{\delta}\right)$ as a function of $\sigma_{\theta}=\sigma_{\delta}=\sigma$, setting $\theta_{0}=\delta_{0}=90^{\circ}$ and $d \theta=d \delta=0.25^{\circ}$ for the numerical integration. We then obtained the average TAA value measured in an experiment resulting from from all three ligands by assuming a 
randomized excited state, such that:

$$
\langle\mathrm{r}\rangle(\sigma, \sigma)=\frac{1}{3}\left(\left\langle\mathrm{r}_{1}\right\rangle(\sigma, \sigma)+\left\langle\mathrm{r}_{2}\right\rangle(\sigma, \sigma)+\left\langle\mathrm{r}_{3}\right\rangle(\sigma, \sigma)\right)
$$

Fig. S.13c displays the obtained conformational averages as a function of the standard deviation $\sigma$. We observe that in an experiment, we would obtain $\langle\mathrm{r}\rangle=-0.1$ for an infinitely narrow conformational ensemble around the octahedral configuration of the tris-chelate complex. Increasing the width of the conformational ensemble for each ligand then leads to a monotonous increase of $\langle\mathrm{r}\rangle$, which reaches a value of approximately -0.05 for $\sigma \approx 30^{\circ}$. Through this calculation we are now able to correlate the initially measured anisotropy of the HS state with the standard deviation $\sigma$ of the conformational ensemble of its ligand system around an octahedral configuration. Recalling the experimentally determined values $\mathrm{r}_{0}^{\mathrm{CIP}}=-0.96, \mathrm{r}_{0}^{\mathrm{SIP}}=-0.87$, and $\mathrm{r}_{0}^{\mathrm{RIP}}=-0.66$, we obtain $\sigma^{\mathrm{CIP}} \approx 7^{\circ}, \sigma^{\mathrm{SIP}} \approx 13^{\circ}$, and $\sigma^{\mathrm{RIP}} \approx 22^{\circ}$. Fig. S.13d then plots one-dimensional Gaussian angular distributions with these standard deviations for a visual comparison. It is likely that the quantitative accuracy of the obtained conformational ensembles is limited due to the simplicity of the presented calculations. Nevertheless, our calculations clearly show that the chiral ion-pairing interaction strongly narrows the conformational distribution of the individual ligands, such that $\mathrm{Fe}^{\mathrm{CIP}}$ is best described as a nearly rigid complex in an octahedral configuration. 

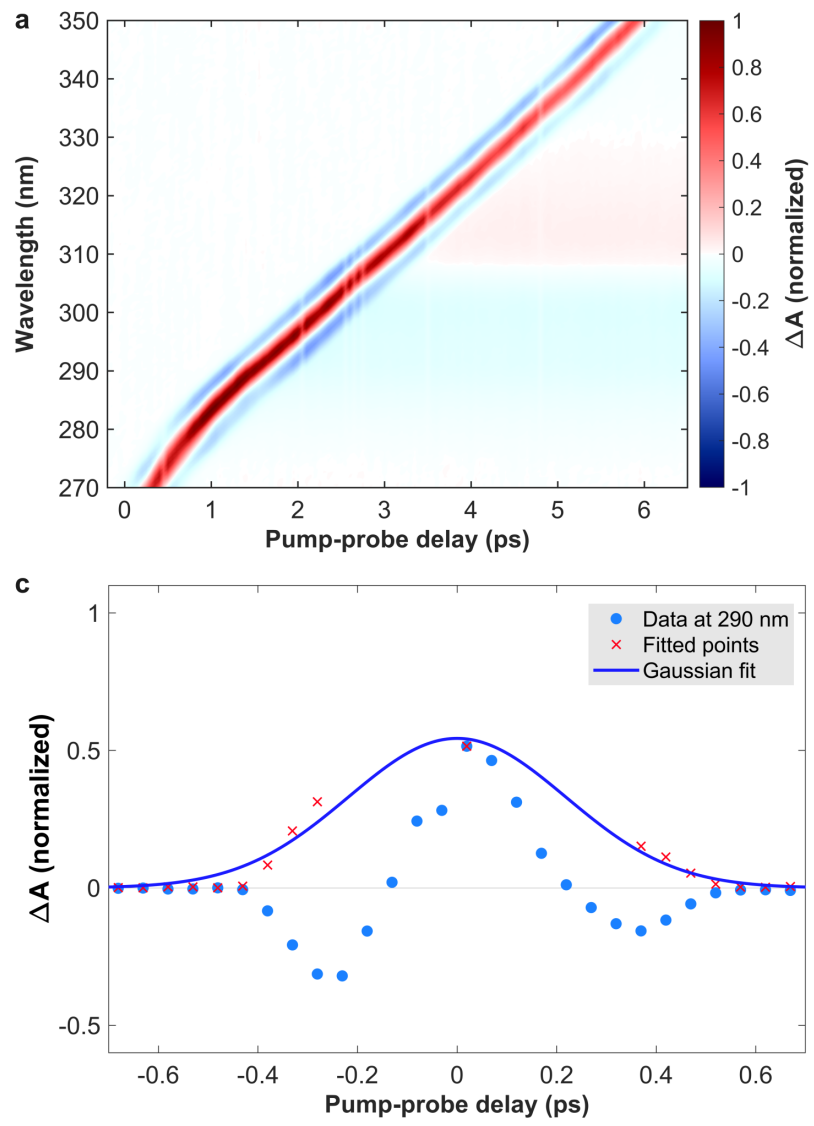

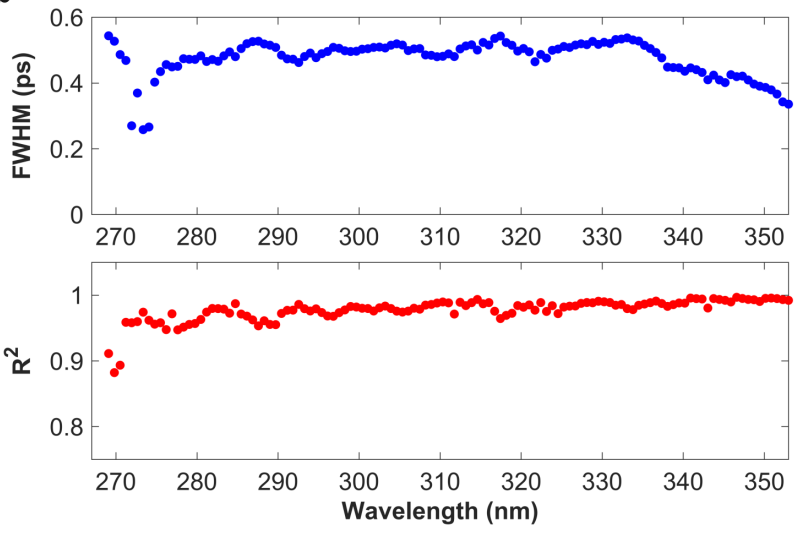

d

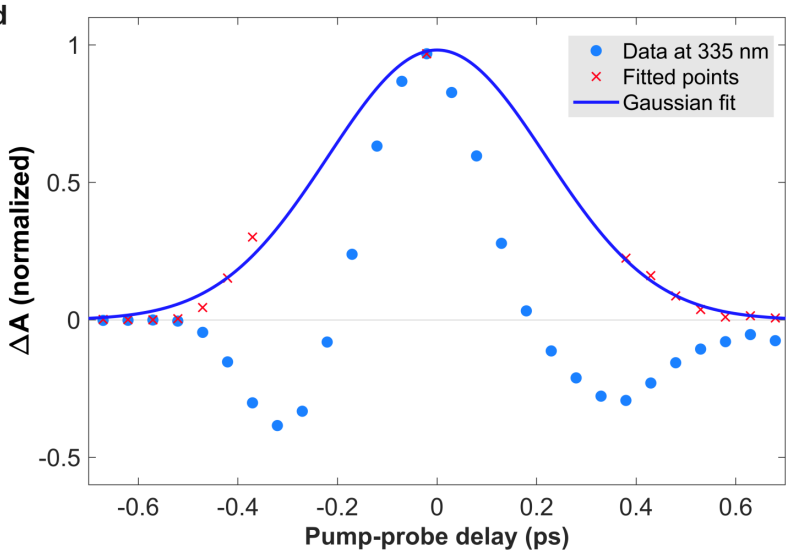

Figure S.14. Transient absorption in pure $\mathrm{CHCl}_{3}$ excited at $530 \mathrm{~mm}$, measured with left-circularly polarized probe pulses in the TRCD setup. a) Time-wavelength map of the obtained data without any chirp correction. b) Results of Gaussian fits of the kinetic traces at individual probe wavelengths, displaying the resulting full-width-at-half-maximum (FWHM) and $\mathrm{R}^{2}$ value. c,d) Selected kinetic traces, selection of data to be fitted and the resulting Gaussian fits.

Instrument response function. The time resolution of the TRCD experiments was determined by recording the instantaneous response of neat $\mathrm{CHCl}_{3}$ in a $0.5 \mathrm{~mm}$ pathlength cell with ultrathin drilled windows (Starna). Fig. S.14a displays the associated TA data, recorded with left-circularly polarized probe pulses and a linearly polarized pump pulse. Due to the large amount of glass in the probe beam path, the GVD of the probe pulses covers approximately $6 \mathrm{ps}$, with the arrival time of each probe wavelength slice taking a nearly linear dependence with respect to the probe wavelength. Note that a very weak, 
long-lived TA signal is observed that resembles the signal from the $\mathrm{Fe}^{\mathrm{II}}$ complexes. This is attributed to minor impurities from the $\mathrm{Fe}^{\mathrm{II}}$ samples remaining in the flow system after completion of the reported experiments. Following the fitting routine applied in section S.2, the IRF of the experiment was estimated via the width of the coherent artifact at each probe wavelength slice. Fig. S.14b displays the FWHM of the associated Gaussian fits as a function of probe wavelength and fig. S.14c,d display example fits at selected probe wavelengths. The performed fits show that the average IRF is approximately 0.5 ps across the probed wavelength window. However, for recording TRCD spectra at a pump-probe delay of $0.5 \mathrm{ps}$, the GVD correction procedure requires a sampling with time steps $<0.5$ ps over the first 6 ps covering the chirp of the probe pulse. Due to the long data acquisition (DAQ) time per data point, a time step of 1 ps was chosen over the duration of the probe pulse chirp instead. After GVD correction, the first TRCD spectrum completely free of contributions from the coherent artifact corresponded to a pump-probe delay of 4 ps.
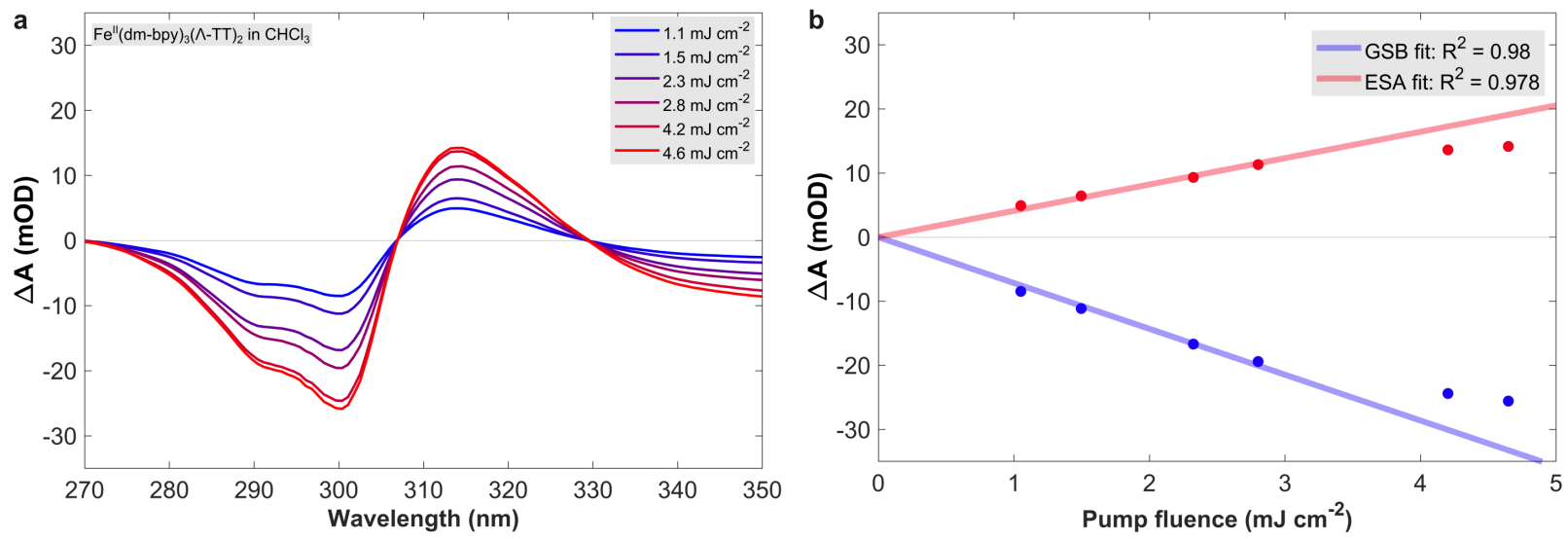

Figure S.15. a) Transient absorption of $\mathrm{Fe}^{\mathrm{II}}(\mathrm{dm}-\mathrm{bpy})_{3}(\Lambda-\mathrm{TT})_{2}$ in $\mathrm{CHCl}_{3}$ as a function of pump fluence at a pump-probe delay of 10 ps. b) Maximum transient absorption amplitude of the GSB and ESA band as a function of pump fluence including a linear fit of the data.

Pump fluence dependence. In order to ensure that the excitation of the MLCT transition takes place in a linear regime with respect to the pump fluence, we recorded TA spectra of $\mathrm{Fe}^{\mathrm{CIP}}$ as a function of pump pulse energy. The pump-probe delay was fixed at $10 \mathrm{ps}$ and the pump pulse energy $E$ was converted to peak fluence $F$ via $F=8 E /\left(w^{2} \pi\right)$, assuming a Gaussian beam with a diameter $w$ at $1 / \mathrm{e}^{2}$ intensity. Here we used an average focal spot diameter of $w=200 \mu \mathrm{m}$. Fig. S.15a displays the acquired spectra, whereas fig. S.15b plots the maximum TA amplitude of the main GSB and ESA band averaged over a $2 \mathrm{~nm}$ wide 
interval as a function of the pump fluence. One clearly observes a saturation of the TA signal for the two highest fluence values. We thus performed a linear fit on the data taking into account only the four lowest fluence values and setting the y-intercept to zero. We obtained an excellent correspondence with the data as evidenced by the high $\mathrm{R}^{2}$ values displayed in the figure. From this data set we estimated the maximum peak fluence in the linear regime to be at $3.5 \mathrm{~mJ} \mathrm{~cm}^{-2}$. For the reported TRCD experiments, we thus employed a pump pulse energy of $1 \mu \mathrm{J}$ at an average focal spot diameter of $270 \mu \mathrm{m}$ at $1 / \mathrm{e}^{2}$ intensity in order to maximize the measured TRCD signals.

Measurement sensitivity. The statistical noise of a TRCD spectrum recorded at a given pump-probe delay mostly depends on the shot-noise of the probe spectrum, the accuracy of its referenced detection and the number of averaged probe shots. For each TRCD experiment we determined the associated standard error at a pump-probe delay of $-0.1 \mathrm{ps}$ (probe pulse arriving $0.1 \mathrm{ps}$ before the pump pulse) as the standard deviation of the measured TRCD amplitude over the number $N$ of the acquired TRCD spectra, divided by $\sqrt{N}$. For the TRCD experiments on the $\Lambda$ configurations $N=800$ and for those on the $\Delta$ configurations $N=600$. Fig. S.16 displays the recorded TRCD spectra with the obtained standard error as a shaded area (second and third column of spectra). The data shows that for all TRCD experiments, baseline deviations from zero are within $\pm 1 \cdot 10^{-5} \mathrm{OD}$, whereas the standard error is $< \pm 1 \cdot 10^{-5} \mathrm{OD}$. However, it is well known that at positive pump-probe delays, the pump-induced anisotropy from the photoexcited molecular ensemble may result in pronounced TRCD artifacts at signal levels exceeding any artifact-free TRCD signals. Similar to TA spectroscopy, a magic angle configuration of the linear pump

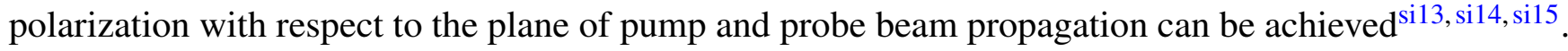
The precise angle depends on the static birefringence in the utilized optical elements and the polarization sensitivity of the optical setup, for example through dispersive optics or polarizers ${ }^{\text {si16, si17, si18 }}$. As these parameters are highly sensitive to the probe beam alignment and environmental conditions such as the ambient temperature, we set the pump polarization prior to every TRCD experiment by minimizing the TRCD signal in the racemic $\mathrm{Fe}^{\mathrm{II}}(\mathrm{bpy})_{3} \mathrm{Cl}_{2}$ in $\mathrm{H}_{2} \mathrm{O}$ at a fixed pump-probe delay of 10 ps. Here any non-zero signal must be attributed to polarization artifacts from pump-induced anisotropy. Fig. S.16 displays the obtained TRCD spectra (solid red lines) associated with each of the reported TRCD experiments (first column of spectra). In all cases, deviations from zero were determined to be within $\pm 1 \cdot 10^{-5} \mathrm{OD}$, which 
TRCD spectroscopy on ultrafast time scales.
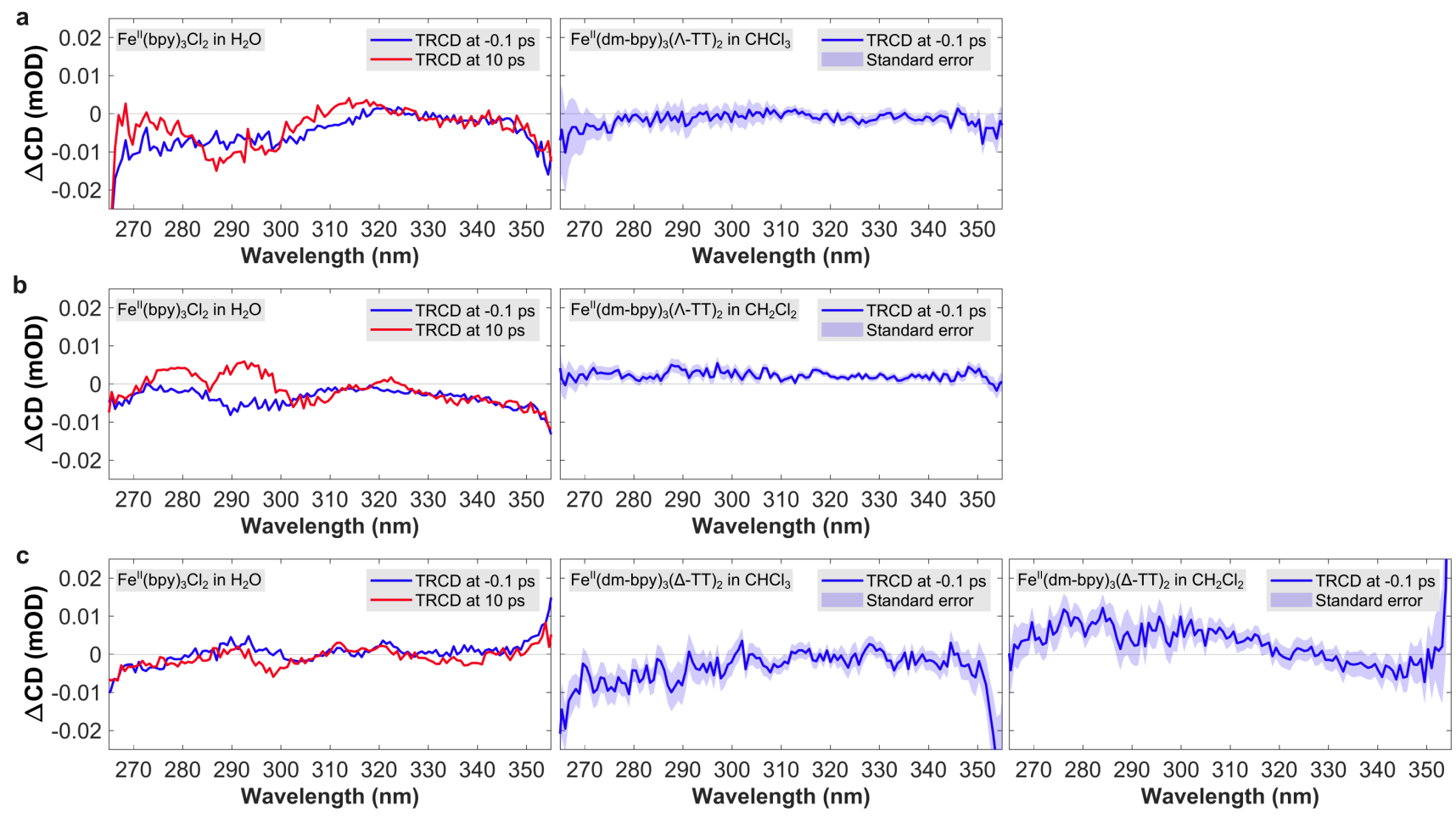

Figure S.16. Assessment of the sensitivity of the TRCD experiments and the suppression of polarization artifacts. The left-most panels display the TRCD spectrum at $-0.1 \mathrm{ps}$ and $10 \mathrm{ps}$ for racemic $\mathrm{Fe}^{\mathrm{II}}(\mathrm{bpy})_{3} \mathrm{Cl}_{2}$ in $\mathrm{H}_{2} \mathrm{O}$, where any non-zero signal is attributed to polarization artifacts. These measurements were performed prior to each of the reported TRCD experiments: a) for $\Lambda-\mathrm{Fe}^{\mathrm{CIP}}$, b) for $\Lambda$-Fe ${ }^{\mathrm{SIP}}$, and c) for $\Delta-\mathrm{Fe}^{\mathrm{CIP}}$ and $-\mathrm{Fe}^{\mathrm{SIP}}$, which were measured back-to-back. The right panels display the TRCD spectra at -0.1 ps for each of the four samples, including the associated standard error as a shaded area.

TRCD data. Fig. S.17 displays the TRCD spectra of $\Lambda, \Delta-\mathrm{Fe}^{\mathrm{CIP}}$ and $-\mathrm{Fe}^{\mathrm{SIP}}$. As indicated in the Methods section, 30,000 probe shots were acquired per pump-probe delay to calculate a TRCD spectrum. For the $\Lambda$ configurations, 30 pump-probe delays were recorded in a scan, which was repeated 800 times. In each scan, the chirp of the probe pulse was sampled with 1 ps steps, such that after GVD correction of the data the earliest recorded pump-probe delay free of any signal from the coherent artifact is 4 ps. Fig. S.17a,c thus displays 26 TRCD spectra with logarithmically increasing pump-probe delay, as indicated in the legend. To check the consistency of the results, the TRCD spectra for the $\Delta$ configurations were 

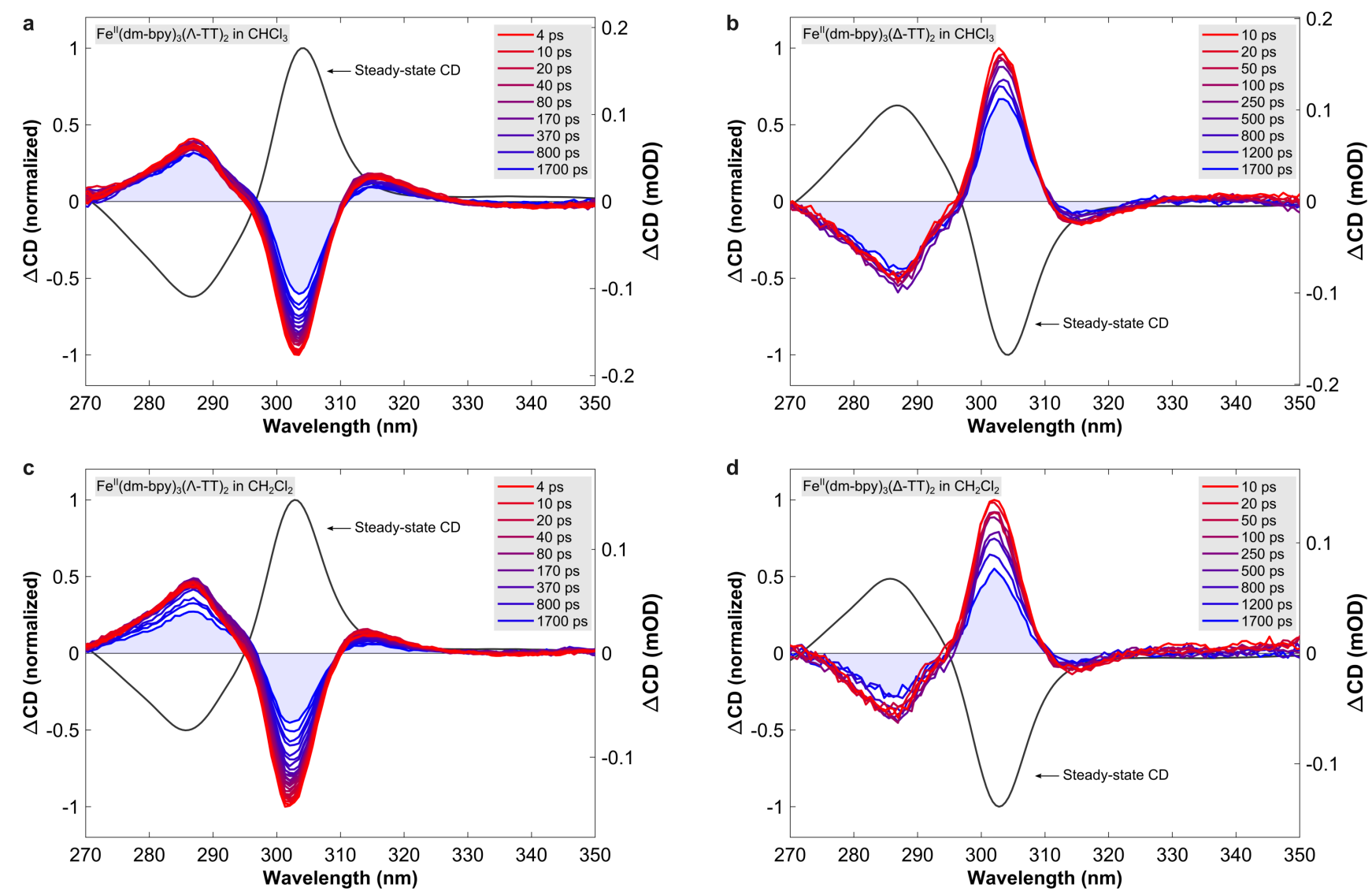

Figure S.17. TRCD spectra as a function of pump-probe delay for $\Lambda-\mathrm{Fe}^{\mathrm{CIP}}(\mathrm{a}), \Delta-\mathrm{Fe}^{\mathrm{CIP}}(\mathrm{b}), \Lambda-\mathrm{Fe}^{\mathrm{SIP}}(\mathrm{c})$, and $\Delta-\mathrm{Fe}^{\mathrm{SIP}}(\mathrm{d})$. For the $\Lambda$ configurations a total of 26 pump-probe delays are displayed, whereas 9 pump-probe delays are displayed for the $\Delta$ configurations. For all samples, the maximum absorbance near $295 \mathrm{~nm}$ was approximately $0.7 \mathrm{OD}$ in a $0.5 \mathrm{~mm}$ pathlength flow cell. The samples were photoexcited at $530 \mathrm{~nm}$ with a peak fluence of approximately $3.5 \mathrm{~mJ} \mathrm{~cm}^{-2}$.

recorded at 10 pump-probe delays over 600 scans. Fig. S.17b,d displays the TRCD spectra for the 9

acquired positive pump-probe delays corresponding to the values indicated in the legend. Note that the earliest pump-probe delay was 10 ps with respect to the coherent artifact at $270 \mathrm{~nm}$, such that no GVD correction was performed. For all four samples, the TRCD spectra acquired at a negative pump-probe delay are displayed in fig. S.16. Starting with $\Lambda-\mathrm{Fe}^{\mathrm{CIP}}$, three bands can be observed: a positive GSB band centered at $285 \mathrm{~nm}$, a negative GSB band centered near $305 \mathrm{~nm}$, and a positive excited state CD band centered near $315 \mathrm{~nm}$. Importantly, the two GSB bands do not display the same decay dynamics, which suggests that they are overlapping with an additional excited state $\mathrm{CD}$ band. $\Lambda-\mathrm{Fe}^{\mathrm{SIP}}$ displays the same GSB and excited state CD bands, but with significantly faster decay dynamics, which also results in an observable decay of the GSB band at $285 \mathrm{~nm}$. Despite its slightly reduced signal-to-noise and less 
recorded pump-probe delays overall, the TRCD spectra for $\Delta-\mathrm{Fe}^{\mathrm{CIP}}$ and $\mathrm{Fe}^{\mathrm{SIP}}$ clearly display the same bands and decay dynamics as their counterparts, yet with an inverted sign, due to the inverted chiral symmetry. As TRCD spectroscopy is notoriously sensitive to polarization artifacts distorting the observed spectral dynamics, this is an important milestone. Together with the absolute sensitivity obtained from racemic samples in fig. S.16, it demonstrates that the reported TRCD experiments are not limited by polarization artifacts such that the observed spectral dynamics can be attributed safely to the molecular dynamics driving the $\mathrm{SCO}$ in the studied $\mathrm{Fe}^{\mathrm{II}}$ complexes.

TRCD fitting procedure and HS CD evolution. In order to develop a global fitting procedure for the TRCD data, we first determined the minimum number of Gaussians that are required to fit a representative TA and TRCD spectrum. To this end, we note that for each pump-probe delay, a TRCD measurement delivers both the TRCD spectrum and TA spectra measured with left- and right-circularly polarized probe pulses. Fig. S.18 displays the obtained data and fits for $\Lambda$-Fe ${ }^{\mathrm{CIP}}$ at a pump-probe delay of 5 ps, whereas table S.4 shows the obtained fit parameters. We found that the TA spectrum can be decomposed into a GSB contribution scaled by an excitation density of $A_{\mathrm{GSB}}=-0.1$ (10\% of the molecules are excited) and a minimum of two Gaussian ESA bands. The $\mathrm{R}^{2}$ value of the fit is excellent and the obtained fit residuals are an order of magnitude lower than the maximum TA signal. We thus attribute the dominant ESA band centered at $299.2 \mathrm{~nm}$ to the LC centered transition of the HS state and note that its spectral shape is well described by a single Gaussian band. For the TRCD spectrum, a successful fit requires two Gaussian HS CD bands, centered at $287.4 \mathrm{~nm}$ and $310.2 \mathrm{~nm}$, respectively, resulting in an excitation density of $12 \%$. This value agrees well with the excitation density obtained from the spectral decomposition of the TA spectrum, as required. Again, the $R^{2}$ value is excellent and the obtained residuals are more than an order of magnitude lower than the maximum TRCD signal. Taking into account the results from the decomposition of the TA spectrum, the obtained results are fully consistent with a scenario where the HS CD is caused by the excitonic coupling of the three LC transitions of the HS state: 1) the sign-pattern of the bisignate CD agrees with the ground state CD, and 2) its zero-crossing near $299 \mathrm{~nm}$ agrees very well with the center of the HS LC band obtained from the spectral decomposition of the TA spectrum. We therefore conclude that the HS CD spectrum is well described by a minimal model consisting of two Gaussian bands with opposite signs. 

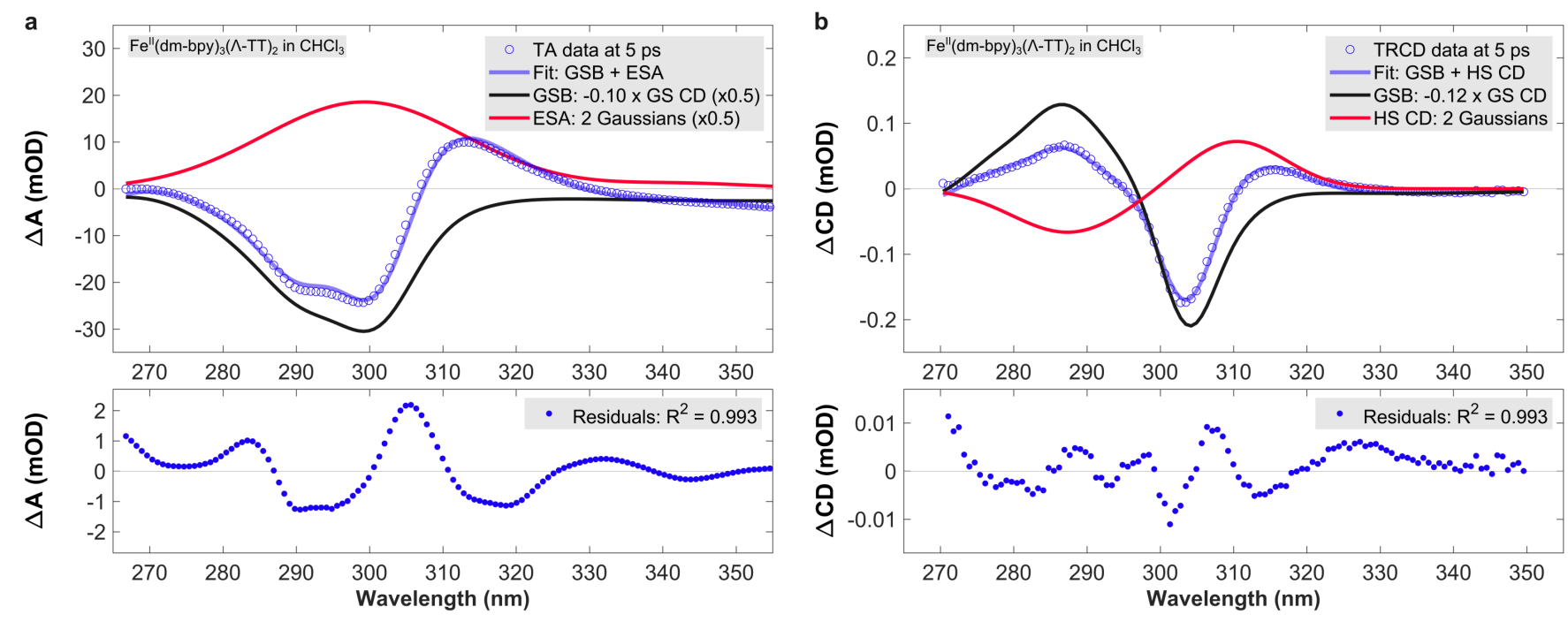

Figure S.18. Spectral decomposition of the simultaneously acquired TA (a) and TRCD (b) spectrum of $\Lambda$-Fe ${ }^{\mathrm{CIP}}$ at 5 ps into a GSB and a HS state contribution consisting of Gaussian bands. Note that in (a) the GSB and ESA bands are scaled by a factor of 0.5 for a better comparison with the TA spectrum.

\begin{tabular}{|l|l|l|l|l|l|l|l|l|}
\hline Spectrum & $\mathrm{R}^{2}$ & $A_{\mathrm{GSB}}$ & $A_{1}(\mathrm{mOD})$ & $\lambda_{1}(\mathrm{~nm})$ & $c_{1}(\mathrm{~nm})$ & $A_{2}(\mathrm{mOD})$ & $\lambda_{2}(\mathrm{~nm})$ & $c_{2}(\mathrm{~nm})$ \\
\hline TA & 0.993 & -0.10 & 37.2 & 299.2 & 19.5 & 2.3 & 342.6 & 14.2 \\
\hline TRCD & 0.993 & -0.12 & -0.067 & 287.4 & 10.9 & 0.073 & 310.2 & 9.2 \\
\hline
\end{tabular}

Table S.4. Fit parameters obtained from the fits displayed in fig. S.18. $A_{n}, \lambda_{n}, c_{n}$ are the amplitude, center wavelength, and width of the Gaussian functions $(n=1,2)$ forming the ESA and HS CD.

At each pump-probe delay t, we can now write the TRCD spectrum as a sum of the GSB and the

HS CD contribution. For this, we assume that the GSB takes the spectral shape of the inverted ground state $\mathrm{CD}$ spectrum $\mathrm{CD}(\lambda)$ scaled by the excitation density $A_{\mathrm{GSB}}$ at the earliest pump probe delay. The time-dependent decay of the GSB is then given by the absolute value of the normalized transient absorption $\left|\mathrm{TA}_{\mathrm{GSB}}(\mathrm{t})\right|$. The HS CD is described by two Gaussian bands, a high-energy band with the subscript $h$ and a low-energy band with the subscript $l$. A priori, all six of the resulting HS CD fit parameters may be time dependent, resulting in the following functional form for a TRCD spectrum:

$$
\operatorname{TRCD}(\lambda, \mathrm{t})=-A_{\mathrm{GSB}}\left|\operatorname{TA}_{\mathrm{GSB}}(\mathrm{t})\right| \mathrm{CD}(\lambda)+A_{h}(\mathrm{t}) \exp \left\{\left(\frac{\lambda-\lambda_{h}(\mathrm{t})}{c_{h}(\mathrm{t})}\right)^{2}\right\}+A_{l}(\mathrm{t}) \exp \left\{\left(\frac{\lambda-\lambda_{l}(\mathrm{t})}{c_{l}(\mathrm{t})}\right)^{2}\right\}
$$

However, the large number of free fit parameters may distort the extracted time-dependent spectral dynamics, if the only optimization criterion is the best fit with the data at each pump-probe delay. In 

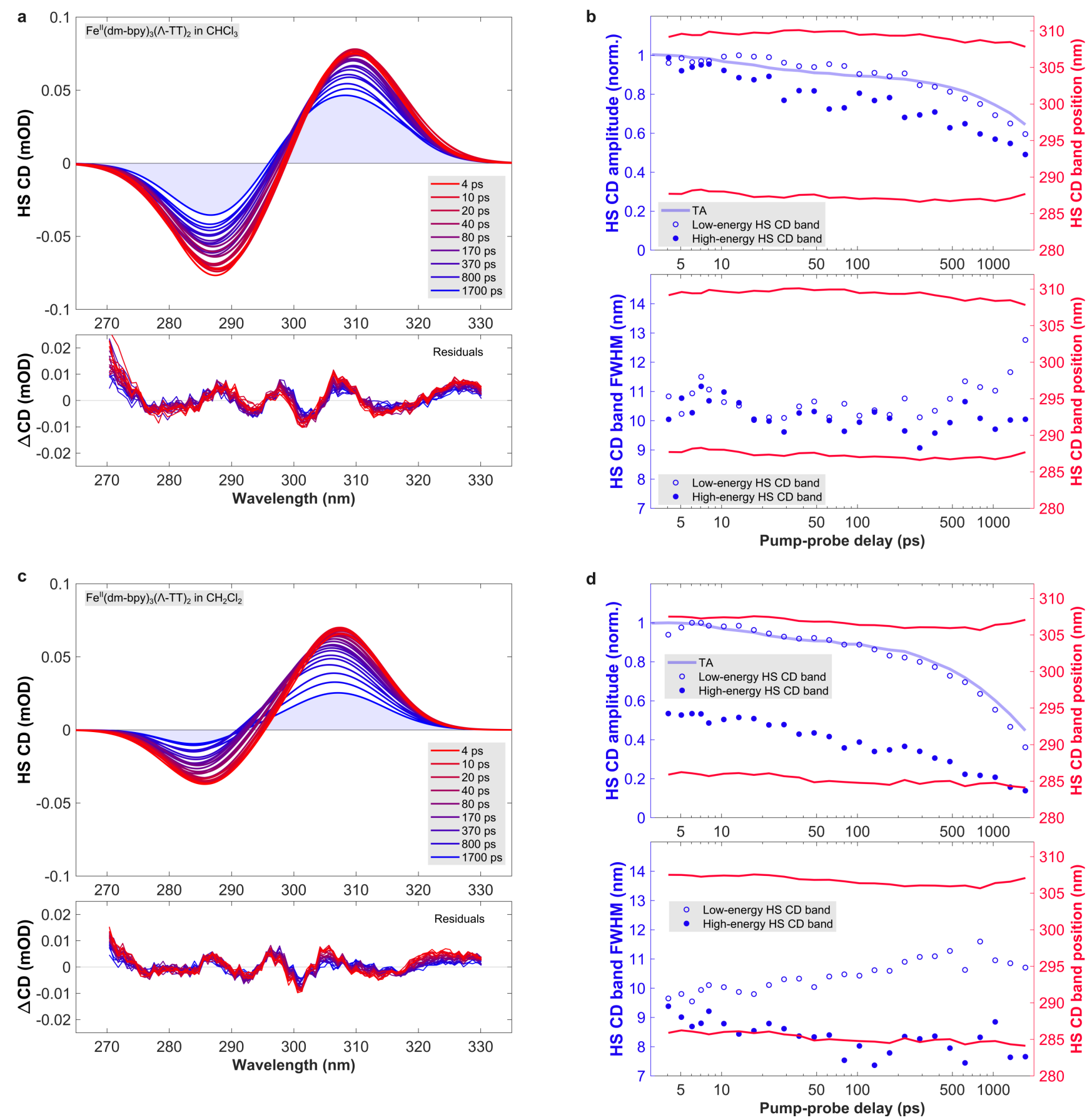

Figure S.19. Time evolution of the HS CD of $\Lambda-\mathrm{Fe}^{\mathrm{CIP}}$ and $-\mathrm{Fe} e^{\mathrm{SIP}}$, obtained from a global fit of the respective TRCD data sets with all six HS CD parameters fit freely for each pump-probe delay. a,b) HS CD spectra, the associated residuals and the obtained HS CD fit parameters as a function of pump-probe delay for $\Lambda-\mathrm{Fe}^{\mathrm{CIP}}$, with $\mathrm{c}$,d) displaying the analogous results obtained for $\Lambda-\mathrm{Fe}^{\mathrm{SIP}}$

practice, this may for example lead to several fit parameters accounting for spectral changes that could

possibly be described accurately by the time-dependent evolution of a single parameter. In order to determine the minimum number of time-dependent fit parameters that are required to achieve a robust 
fit of the recorded data sets, we first applied the developed fit function without any further constraints in order to identify trends in the spectral evolution of the HS CD. To this end, we focused on $\Lambda$-Fe $e^{\mathrm{CIP}}$ and $-\mathrm{Fe}^{\mathrm{SIP}}$, due to their high signal-to-noise and large number of data points. Fig. S.19a,c displays the obtained HS CD spectra as a function of pump-probe delay including their respective residuals, whereas S.19b,d displays the obtained fit parameters as a function of pump-probe delay. First of all we note that for both data sets the residuals are found to be within $\pm 1 \cdot 10^{-5}$ OD for all pump-probe delays, which implies an excellent correspondence between the fit and the data. The evolution of the HS CD spectra of $\Lambda$-Fe $\mathrm{CIP}_{i}$ dominated by an asymmetric decay of the two HS CD bands: at the earliest pump-probe delay, the absolute amplitudes are mostly identical, whereas at the latest delay, the high-energy band has a significantly lower amplitude. Whilst the positions of the bands vary with pump-probe delay, a clear trend in their evolution is not observed. These observations are supported by the time evolution of the individual fit parameters displayed in fig. S.19b, where the HS CD band amplitudes were normalized to the maximum absolute value for better comparison. Whilst the amplitude of the low-energy HS CD band decays mostly like the TA signal (solid line), the high-energy band amplitude decays more strongly. The widths and positions of the two bands do not follow an observable trend, which suggests that these parameters may be suitable time independent constants in a constrained model fit.

In direct comparison, the spectral evolution of the HS CD spectrum of $\Lambda$-Fe ${ }^{S_{P}}$ in fig. S.19c is more complex. The band amplitudes show a similar yet more pronounced evolution: at the earliest pump-probe delay, the high-energy band is already significantly weaker than the low-energy counterpart and it decays more strongly with increasing pump-probe delay. Furthermore the low-energy band displays a significant broadening at later times. Again, the time evolution of the individual fit parameters displayed in fig. S.19d supports these observations. Whilst the low-energy band amplitude follows the decay of the TA, the high-energy band amplitude displays different decay dynamics, which results in a significant reduction of the band amplitude ratio $A_{h} / A_{l}$. In addition, we now find a pronounced increase in the width of the low-energy band and a minor decrease in the band positions with increasing pump-probe delay. This suggests that in the case of $\Lambda-\mathrm{Fe}^{\mathrm{SIP}}$ at least one additional time-dependent parameter is required together with the band amplitudes in order to describe the spectral evolution of the HS CD. However in both cases an asymmetric decay of the band amplitudes is observed to be the dominant effect. 

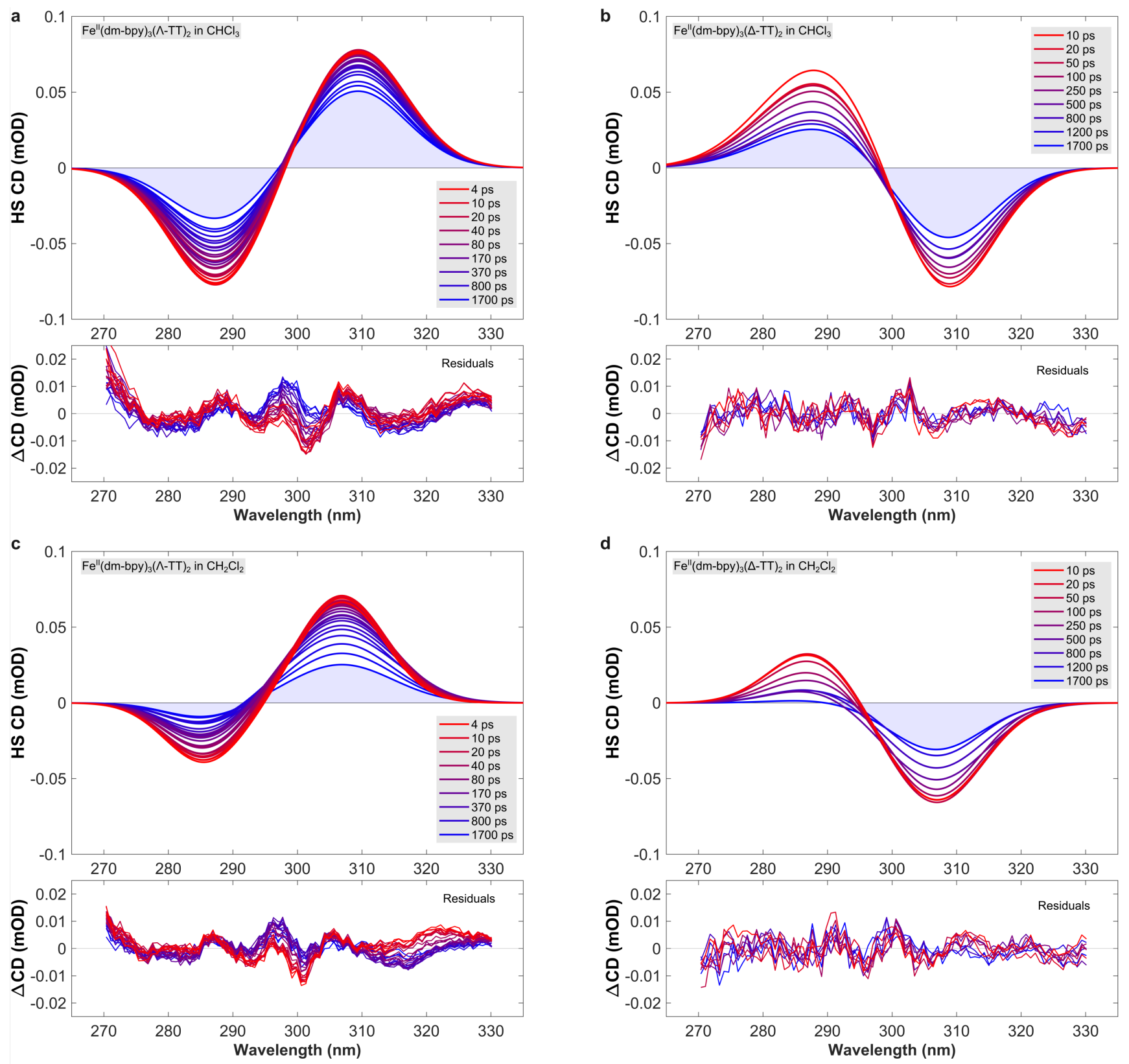

Figure S.20. Time evolution of the HS CD of $\Lambda, \Delta-\mathrm{Fe}^{\mathrm{CIP}}$ and $-\mathrm{Fe} \mathrm{SIP}^{\mathrm{S}}$, obtained from a constrained global fit of the respective TRCD data sets, as explained in the text. a,b) HS CD spectra and the associated residuals for $\Lambda, \Delta-\mathrm{Fe}^{\mathrm{CIP}}$, with $\mathrm{c}, \mathrm{d}$ ) displaying the analogous results obtained for $\Lambda, \Delta-\mathrm{Fe}^{\mathrm{SIP}}$.

In the following we performed fits using the constrained fit functions proposed above. For Fe ${ }^{\mathrm{CIP}}$ in both its $\Lambda$ and $\Delta$ configuration we obtained robust fits with $A_{h}(\mathrm{t})$ and $A_{l}(\mathrm{t})$ as the only time-dependent fit parameters. The remaining parameters $A_{\mathrm{GSB}}, \lambda_{h}, \lambda_{l}, \mathrm{c}_{h}$ and $\mathrm{c}_{l}$ were assumed to be constant for all 1073 pump-probe delays and were thus fit globally across each data set. In the case of $\mathrm{Fe}^{\mathrm{SIP}}$, a fit function 1074 with only the amplitudes as free parameters did not converge to a meaningful solution, confirming the 
previously made observation that the spectral dynamics are more complex compared to Fe ${ }^{\mathrm{CIP}}$. We found that robust fits could be achieved with either one of the widths as an additional time dependent parameter, with the solution employing $c_{l}(\mathrm{t})$ resulting in more robust fits across both the $\Lambda$ and $\Delta$ configurations. Whilst we cannot exclude that minor a time-dependent evolution of the HS CD band positions may take place in $\Lambda, \Delta-\mathrm{Fe}^{\mathrm{SIP}}$, we note that it is not required for an accurate description of the data. We further stress that the observed asymmetric decay of the band amplitudes remains the dominant effect in the HS CD evolution with any additional change playing a comparatively minor role.

Fig. S.20 displays the time evolution of the HS CD obtained from the constrained fits described above. For the $\Lambda$ configurations of $\mathrm{Fe}^{\mathrm{CIP}}$ and $\mathrm{Fe}^{\mathrm{SIP}}$ we qualitatively observe the same spectral evolution as for the fit function with free fit parameters, demonstrating that the additional constraints do not distort the extracted HS CD dynamics. This is supported by the obtained residuals that display almost identical amplitudes within the range of $\pm 1 \cdot 10^{-5} \mathrm{OD}$, albeit with a minor time dependence. This shows that the additional constraints do not compromise the accuracy of the performed fits. As expected, the HS CD dynamics obtained for the $\Delta$ configurations display the same spectral evolution as their counterpart but with an inverted sign. Nevertheless, minor quantitative differences can be observed, which are assessed more closely in fig. S.21. Starting with $\mathrm{Fe}^{\mathrm{CIP}}$, one observes that the band positions obtained for the $\Lambda$ and $\Delta$ configurations agree within $\pm 1.3 \mathrm{~nm}$. Concerning the widths, the values obtained for $c_{l}$ agree very well, whereas the values for $c_{h}$ differ by about $20 \%$. For the amplitudes, $A_{l}(\mathrm{t})$ follows the TA in both data sets, whereas $A_{h}(\mathrm{t})$ is shifted to lower values by about $20 \%$. Whilst these are non-negligible quantitative deviations, we note that they do not have any impact on the interpretation of the experiments. In the case of $\mathrm{Fe}^{\mathrm{SIP}}$, the fit parameters obtained for the $\Lambda$ and $\Delta$ configurations display an excellent correspondence. The only minor disagreement can be seen in $c_{l}(\mathrm{t})$ for the $\Delta$ configuration, which does not display a clear monotonous increase for larger pump-probe delays. We thus conclude that the spectral dynamics of the HS CD are well described by the constrained fit models, which lead to robust and consistent results across all data sets.

In order to quantify the dynamics encoded in the HS CD amplitude decays, we performed multiexponential fits on the values obtained for $A_{h}(\mathrm{t})$ and $A_{l}(\mathrm{t})$ from the constrained HS CD model fit. Fig. S.21 indicates that their time evolution contains the time-dependent decay of the the excited state given 

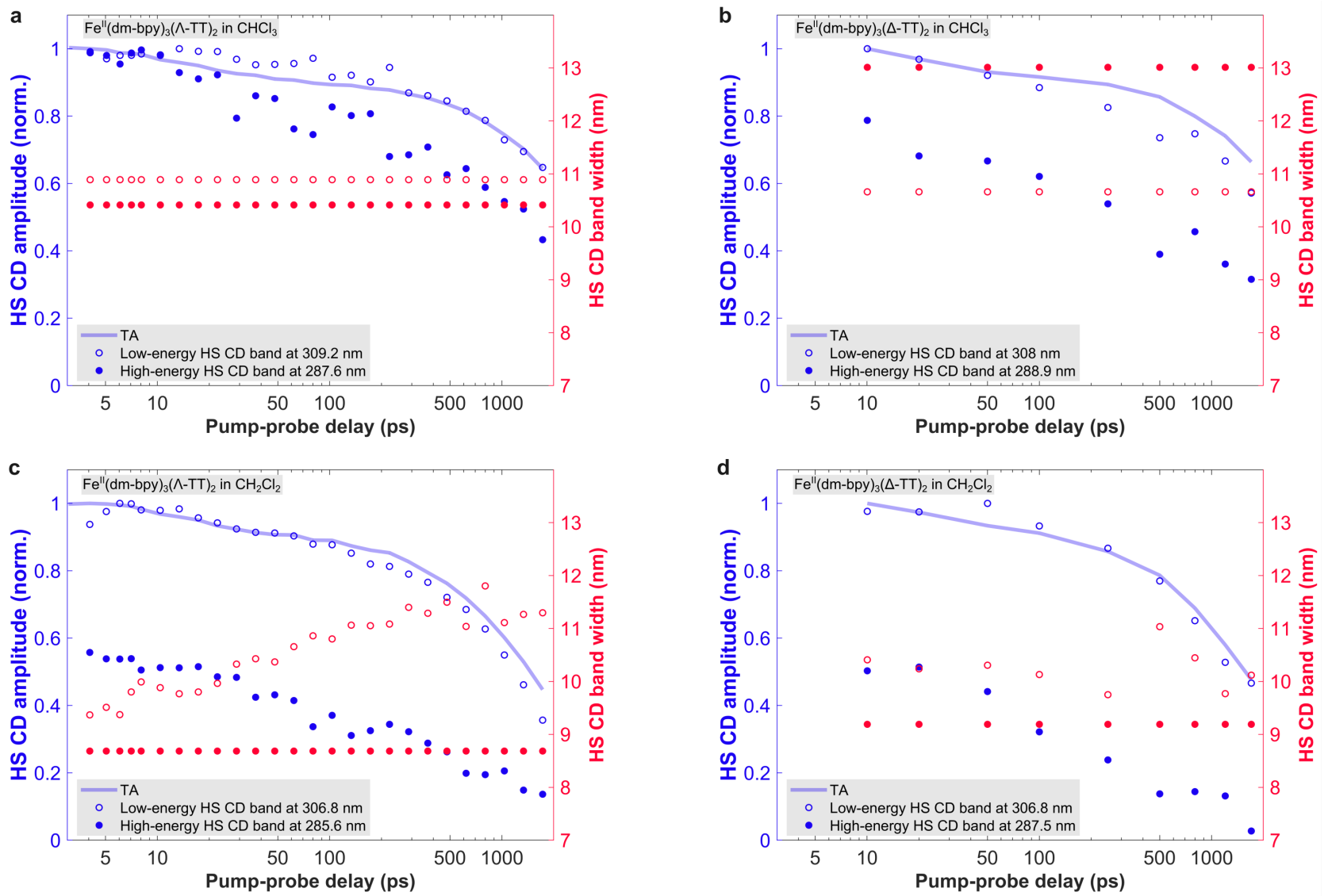

Figure S.21. Time evolution of the HS CD fit parameters of $\Lambda, \Delta-\mathrm{Fe}^{\mathrm{CIP}}$ and $-\mathrm{Fe}^{\mathrm{SIP}}$, obtained from a constrained global fit of the respective TRCD data sets, as explained in the text. a,b) Fit parameters for $\Lambda, \Delta-\mathrm{Fe}^{\mathrm{CIP}}$, with c,d) displaying the analogous results obtained for $\Lambda, \Delta-\mathrm{Fe}^{\mathrm{SIP}}$.

by the TA dynamics. This is consistent with the fact that the HS CD must decay globally with the HS state population. However, the more pronounced decay of $A_{h}(\mathrm{t})$ suggests the presence of at least one additional decay component. On this basis, we used a fit function consisting of a sum of two exponential decays, with one time constant fixed as $\tau_{3}$, the time constant associated with the electronic decay of the HS state, obtained from the TA experiments. For each $\Lambda-\mathrm{Fe}^{\mathrm{CIP}}$ and $-\mathrm{Fe}^{\mathrm{SIP}}$, we simultaneously fitted $A_{h}(\mathrm{t})$ and $A_{l}(\mathrm{t})$ with this fit function, with fig. S.22 displaying the obtained fits and their residuals. We observe an excellent agreement between the fit and the data, which is further supported by the low amplitude of the residuals. The obtained fit parameters are displayed in table S.5. We found that the additional decay components $\phi^{\mathrm{CIP}}=80 \pm 10$ and $\phi^{\mathrm{SIP}}=70 \pm 10$ agree within their error ranges. However, whilst their contribution to the decay of $A_{l}(\mathrm{t})$ is about $10 \%$ for both samples, their contribution to $A_{h}(\mathrm{t})$ is significantly more pronounced and displays a strong dependence on the ion-pairing interaction. In $\Lambda$-Fe ${ }^{\mathrm{CIP}}, \phi^{\mathrm{CIP}}$ 
accounts for $28 \%$ of the decay of $A_{h}(\mathrm{t})$, whereas in $\Lambda-\mathrm{Fe}^{\mathrm{SIP}}$, this contribution rises to $43 \%$.

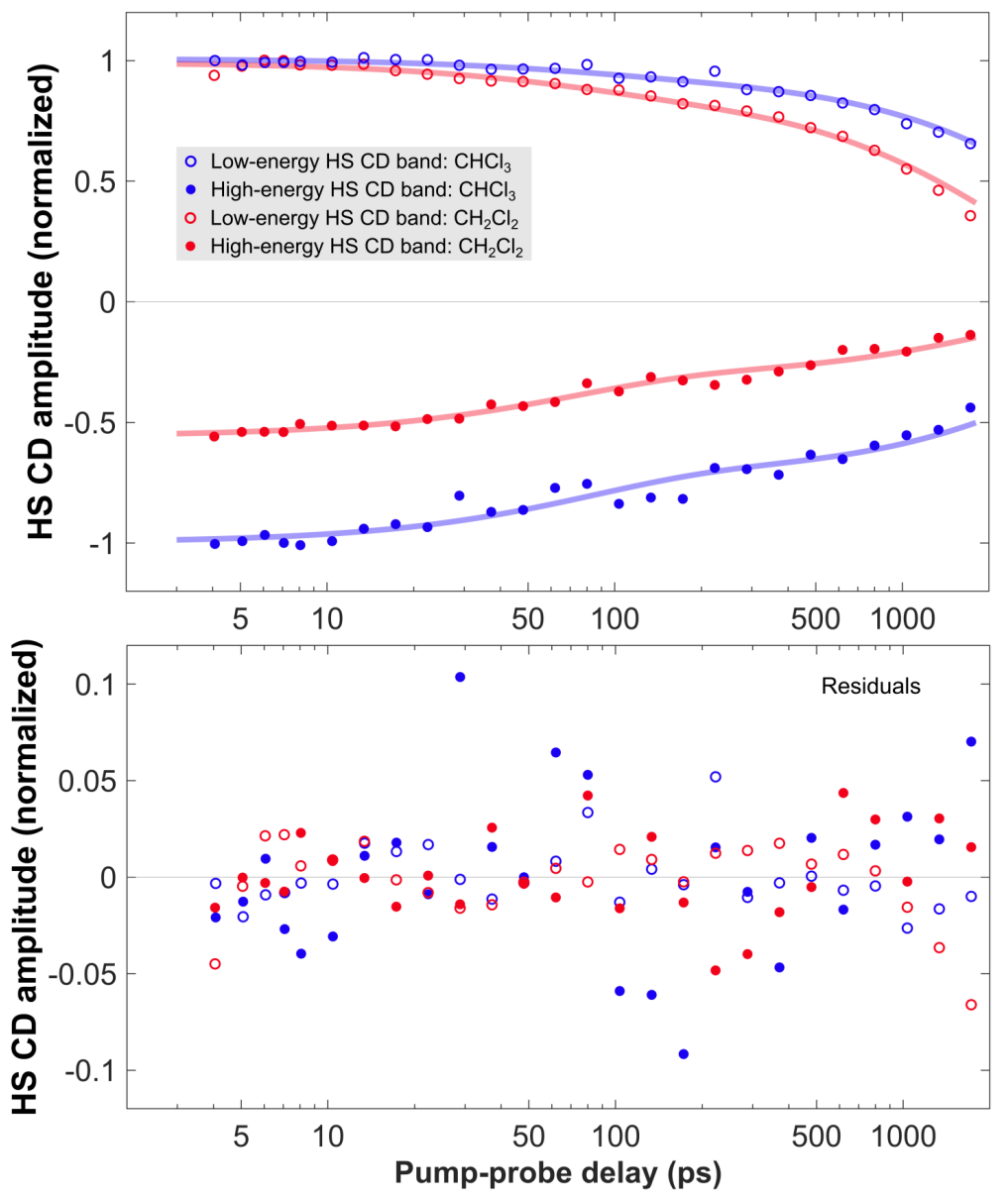

Figure S.22. Time evolution of the high- and low-energy HS CD band amplitudes for $\Lambda$-Fe ${ }^{\mathrm{CIP}}$ and $-\mathrm{Fe}^{\mathrm{SIP}}$. The solid lines display the multi-exponential fits applied to the data points with the obtained fit parameters displayed in table S.5.

\begin{tabular}{|l|l|l|l|l|l|l|}
\hline Sample & $\phi(\mathrm{ps})$ & $A_{l}^{\phi}$ & $A_{h}^{\phi}$ & $\tau_{3}(\mathrm{ps})$ & $A_{l}^{\tau}$ & $A_{h}^{\tau}$ \\
\hline$\Lambda-\mathrm{Fe}^{\mathrm{CIP}}$ & $80 \pm 10$ & 0.07 & -0.28 & 4970 (fixed) & 0.94 & -0.72 \\
\hline$\Lambda-\mathrm{Fe}^{\mathrm{SIP}}$ & $70 \pm 10$ & 0.12 & -0.24 & 2370 (fixed) & 0.88 & -0.32 \\
\hline
\end{tabular}

Table S.5. Fit parameters obtained from simultaneously fitting a sum of two exponential functions to the time evolution of the high- and low-energy HS CD band amplitudes of $\Lambda-\mathrm{Fe}^{\mathrm{CIP}}$ and $-\mathrm{Fe}^{\mathrm{SIP}}$.

References Supporting Information

[si1] Mason, S. F., Peart, B. J. \& Waddell, R. E. Optical rotatory power of co-ordination compounds. Part XVI. Intermediate exciton coupling in the circular dichroism of trisbipyridyl complexes. $J$. Chem. Soc. Dalton Transactions 0, 944-949, DOI: 10.1039/DT9730000944 (1973). Publisher: Royal Society of Chemistry. 
[si2] Castellucci, E., Salvi, P. R. \& Foggi, P. Two-photon excitation spectra of the lowest electronic states of 2,2'-bipyridine. Chem. Phys. 66, 281-291, DOI: 10.1016/0301-0104(82)88027-0 (1982).

[si3] Gawelda, W. et al. Ultrafast Nonadiabatic Dynamics of [FeII(bpy)3]2+ in Solution. J. Am. Chem. Soc. 129, 8199-8206, DOI: 10.1021/ja070454x (2007).

[si4] Decurtins, S., Felix, F., Ferguson, J., Guedel, H. U. \& Ludi, A. The electronic spectrum of tris(2,2'-bipyridine)iron(2+) and tris (2,2'-bipyridine)osmium(2+). J. Am. Chem. Soc. 5 (1980).

[si5] Mason, S. F. The electronic spectra and optical activity of phenanthroline and dipyridyl metal complexes. Inorganica Chimica Acta Rev. 2, 89-109, DOI: 10.1016/0073-8085(68)80016-6 (1968).

[si6] Bosnich, B. Application of exciton theory to the determination of the absolute configurations of inorganic complexes. Accounts Chem. Res. 2, 266-273, DOI: 10.1021/ar50021a002 (1969).

[si7] Ruckebusch, C., Sliwa, M., Pernot, P., de Juan, A. \& Tauler, R. Comprehensive data analysis of femtosecond transient absorption spectra: A review. J. Photochem. Photobiol. C: Photochem. Rev. 13, 1-27, DOI: 10.1016/j.jphotochemrev.2011.10.002 (2012).

[si8] Graener, H., Zürl, R. \& Hofmann, M. Vibrational Relaxation of Liquid Chloroform. The J. Phys. Chem. B 101, 1745-1749, DOI: 10.1021/jp9624212 (1997). Publisher: American Chemical Society.

[si9] Consani, C. et al. Vibrational Coherences and Relaxation in the High-Spin State of Aqueous [FeII(bpy)3]2+. Angewandte Chemie Int. Ed. 48, 7184-7187, DOI: 10.1002/anie.200902728 (2009).

[si10] Auböck, G. \& Chergui, M. Sub-50-fs photoinduced spin crossover in $\left[\mathrm{Fe}(\mathrm{bpy})_{3}\right]^{2+}$. Nat. Chem. 7, 629-633, DOI: 10.1038/nchem.2305 (2015).

[si11] Slavov, C., Hartmann, H. \& Wachtveitl, J. Implementation and Evaluation of Data Analysis Strategies for Time-Resolved Optical Spectroscopy. Anal. Chem. 87, 2328-2336, DOI: 10.1021/ ac504348h (2015).

[si12] Wallin, S., Davidsson, J., Modin, J. \& Hammarström, L. Femtosecond Transient Absorption Anisotropy Study on [Ru(bpy)3]2+ and [Ru(bpy)(py)4]2+. Ultrafast Interligand Randomization of the MLCT State. The J. Phys. Chem. A 109, 4697-4704, DOI: 10.1021/jp0509212 (2005).

[si13] Che, D., Goldbeck, R. A. \& Kliger, D. S. Theory of natural circular dichroism in molecules oriented by photoselection. The J. Chem. Phys. 100, 8602-8613, DOI: 10.1063/1.466714 (1994).

[si14] Cho, M. Two-dimensional circularly polarized pump-probe spectroscopy. The J. Chem. Phys. 119, 7003-7016, DOI: 10.1063/1.1599344 (2003).

[si15] Schott, S., Steinbacher, A., Buback, J., Nuernberger, P. \& Brixner, T. Generalized magic angle for time-resolved spectroscopy with laser pulses of arbitrary ellipticity. J. Phys. B: At. Mol. Opt. Phys. 47, 124014, DOI: 10.1088/0953-4075/47/12/124014 (2014).

[si16] Xie, X. \& Simon, J. D. Picosecond circular dichroism spectroscopy: a Jones matrix analysis. $J$. Opt. Soc. Am. B 7, 1673, DOI: 10.1364/JOSAB.7.001673 (1990).

[si17] Bjorling, S. C. et al. Analysis of optical artifacts in ellipsometric measurements of time-resolved circular dichroism. The J. Phys. Chem. 95, 4685-4694 (1991).

[si18] Oppermann, M. et al. Ultrafast broadband circular dichroism in the deep ultraviolet. Optica 6, 56-60, DOI: 10.1364/OPTICA.6.000056 (2019). 